\title{
GÉOMÉTRIE NON EUCLIDIENNE
}

PA R

P. BARBARIN,

PROPESEEUR A V LYCRE TF RORIEAUX.

DEUXIEME EDITION.

Scientia, $\mathbf{n}^{\circ} 15$.

IRIS - LILLIAD - Université Lille 1 
IRIS - LILLIAD - Université Lille 1 


\section{TABLE DES MATIÈRES.}

\section{CHAPITRE PREMIER. \\ Considérations générales et historiques.}

1. Euclide. ................................

2. Premières idées touchant la Géomètrie non euclidienne..... o

3. Les fondateurs de la Géometrie non euclidienne. Lobatschewsky, Bolyai, Riemann. Leurs continuateurs........ so

CHAPITRE 11.

Les définitions et postulats d'après Euclido.

Les trois Géométries.

4. Les défnitions............................

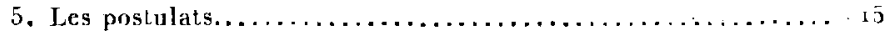

6. Les définitions de la droite et du plan................. I6

7. Programme des principales propositions élémentaires de la

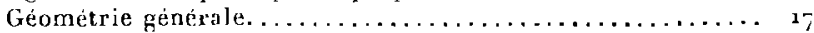

8. Les hypothèses de Saccheri. ................. I8

9. Région normale............................

10. Extension de la région normale.................. 2 I

11. Hypothèse de l'angle droit. Géométrie euclidienne......... 2r

12. Hypothèse de J'angle aigu. Géométrie lobatschewskienne..... 22

13. Hypothèse de l'angle obtus. Gèmétrie riemannienne....... 25

14. Etude inverse.............................. 28

15. Le plan elliptique de Cayley-Klein................. $3_{3}$

16. Les Géométries non archimédiennes................ 33

\section{CHAPITRE III}

La distance comme notion fondamentale.

17. Les travaux de De Tilly .................... 35

18. La droile et le plan d'après Cauchy ............... 38

IRIS - LILLIAD - Université Lille 1 


\section{CHAPITRE IY. \\ La Góométrie générale dans le plan et dans l'espace.}

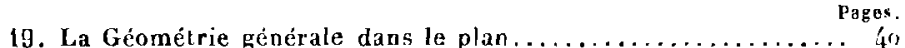

19. La Geométrie genérale dans le plan................

20. La Géométrie générale dans l'espace............... 4́f

21. Théorie des droites et plans qui ont une normale commune.. 46

22. Théorie des droites et plans parallèles............... 47

\section{CHAPJTRE V.}

\section{La Trigonométrie.}

23. Formules des triangles...................... 49

24. Formules des quadrilatères. Constructions fondamentales.... 55

\section{CHAPITRE VI. \\ Mesure des aires et volumeg.}

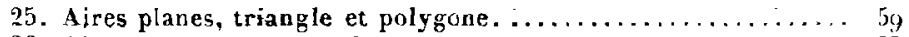

26. Aires des surfaces courbes....................... 6.5

27. Volumes. . . . . . . . . . . . . . . . . . . . . . 65

\section{CHAPITRE VII.}

Les contradicteurs de la Géométrie non euclidienne.

28. Objections principales....................

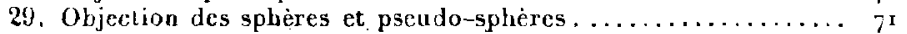

30. Objection du triangle équilatéral.................... $7^{6}$

31. Autres objections ........................ 77

32. L'impossibilité de démontrer le Postulatum d'Euclide...... 79

\section{CHAPITRE VIII.}

\section{La Géométrie physique.}

33. La forme géométrique de notre univers............. 8,

34. Mesures relatives au paramètre................... 83

Note. - Sur deux quadrilatères birectangles et isoscèles de la région normale....................... $8_{7}$

\section{PLANCHES.}

Fac-similé des Éléments d'Euclide.................. I't

l'ortrait de Lobatschewsky........................

Portrait de Bernhard Riemann..................... ${ }_{2} 6$

IRIS - LILLIAD - Université Lille 1 


\title{
LA GÉOMÉTRIE NON EUCLIDIENNE.
}

\author{
- La découverte de la Géométrie \\ y non euclidienne, vers 1830 , étai \\ - inévitable. \\ HA1STED.
}

\section{CHAPITRE PREMIER.}

CONSIDÉRATIONS GÉNÉRALES ET HISTORIQLES.

1. Euclide ( $\left.{ }^{1}\right)$ - A l'immortel Euclide revient la gloire d'avoir définitivement tixé, dans un Livre qui fait encore autorité dans l'enseignement et que l'on ne saurait trop étudier et approfondir, les principes de la Géométrie usuelle. D'après Hérodote et Aristote, c'est en Égypte, sur les bords du Nil, que cette Science prit naissance, au temps de Sésostris ( $\left.{ }^{2}\right)$. D'Égypte, elle passa en Grèce, grâce à Thalès, Pythagore, Platon et leurs disciples $\left({ }^{3}\right)$; mais c'est encore en Égypte, à Alexandrie, que nous la retrouvons magnifiquement enseignée par Euclide au sein de la célèbre École

( $\left.{ }^{1}\right)$ Génmètre gres, vers 320 avant notre ère.

$\left({ }^{2}\right)$ " Quand une inondation du Nil enlevait a quelqu'un une partie de son lol, il allait exposcr à Sésostris la perte qu'il avait subie, et le roi mandait des géomètres chargés de mesurer l'étendue du dommage; de cetle façon, la redevance convenue n'était payée que pour le terrain restant. " (HERoDote, Livre IL, § 109 .)

$\left({ }^{3}\right)$ On sait que la tradition attribue a Thalès la valeur égale à deux droits de la somme des angles d'un triangle, et la proportionnalité des cótés homologues dans deux triangles où les angles homologues sont respectivement égaux. 
fondée par le géomètre grec dans cette ville sous la protection de P'tolémée, École qui devait nous donner aussi Archimède et Apollonius.

C'est donc l'expérience qui a fourni anx géomètres anciens un certain nombre de notions primitives, d'axiomes, ou de postulats fondamentaux mis par eux à la base de la Science pour en diriger et assurer les déductions; peut-être les prédécesseurs d'Euclide en admettaient-ils un nombre surabondant, mais, dès l'époque d'Wuclide, ce nombre est ramené au strict minimum nécessaire, et tous les autres non compris dans cette liste pouvant se démontrer, sont mis au rang de théorèmes.

Les Éléments de Géométrie d'Euclide ont joui pendant tout le moyen àge et jouissent encore d'une célébrité qu'aucun Ouvrage de Science n'a pu atteindre; cette célébrité est due à leur perfection logique, perfection que nous mettrons en relief au cours de notre étude, à l'admirable enchaìnement des propositions, et à la rigueur des démonstrations, "Il mit dans son Livre, dit Montucla, cet enchainement si admiré par les amateurs de la rigueur géométrique... En vain, ajoutet-il, divers géomètres, à qui cet arrangement a déplu, ont tâché de le réformer. Lêurs efforts impuissants ont fait voir combien il était difficile de substituer à la chaîne formèe par le gcomètre grec une autre aussi ferme et aussi solide."

2. Premières idées touchant la Géométrie non euclidienne. - Cette opinion de l'historien des Mathématiques conserve toute sa valeur devant les recherches que les géomètres ont entreprises depuis un siècle environ à l'effet de soumettre les principes fondamentaux de la Science à un examen raisonné et approfondi. Les postulats d'Euclide sont absolument rigoureux, et, en pratique, notre expérience ne peut, jusqu'ici du moins, contredire aucune de leurs conséquences, tellement ils sont bien choisis, comme nous le verrons, pour l'objet du géomètre grec. Mais l'idée devait fatalement naître un jour, chez un esprit critique et original, de se demander ce qui arriverail si tel d'entre eux n'était pas vrai ou était remplacé par un postulat plus général. C'est cette idée même qui, aux environs de 1813 , avait conduit plusieurs géomètres à concevoir presque simultanément une 


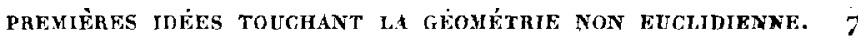

Géométrie nommée par eux tantôt anti-euclidienne, tantôt astrale, tantòt enfin non euclidienne, c'est-à-dire une Géométrie de laquelle la célèbre proposition connue ordinairement sous le nom de Postulatum d'Euclide se trouve écartée. Quelques courtes notions historiques sont ici nécessaires au lecteur pour comprendre l'évolution qui devait présider à la création de la nouvelle Gremétrie ( $\left.{ }^{1}\right)$. Quand on aborde la théorie des parallèles par quelque còté que ce soit, existence de la parallèle unique, position relative de deux droites qui forment certains angles avec unc autre, somme des angles d'un triangle, droites équidistantes, etc., on vient se heurter à une proposition qui ne peut se deduire des précédentes et que l'on est obligé d'admettre sans démonstration. Ainsi, dans ses Éléments, Euclide demande qu'on lui accorde que: si deux droites situées dans un même plan font avec une sécante, et d'un même cóté de celle-ci, des angles intérieurs dont la somme est maindre que deux droits, ces droites prolongées suffisamment se rencontrent de ce cóté. Euclide a donc très bien vu les difficultés cachées qui existent dans la theorie des paralléles; le texte de son postulat, la place même qu'il lui donne prouvent surabondamment combien il avait réfléchi sur les origines de la Géométrie. Il n'est pas impossible que le géomètre grec ait examiné un instant l'hypothèse contraire, dans laquelle les deux droites précédemment énoncées ne se rencontrent pas nécessairement, et qu'il ne l'ait rejetée qu'à bon escient, à cause de sa complication apparente; quoi qu'il en soit, son postulat n'a d'autre valeur à ses yeux qu'une hy pothèse; sans cela, nous n'en pouvons douter, il eùt formulé sa proposition dans d'autres termes et essayé tout au moins de la démontrer.

Pourtant, depuis Euclide jusqu'à Legendre, c'est-à-dire pendant plus de deux mille ans, les géomètres en ont méconnu la vraie nature et, supposant à tort qu'elle est contenue dans la notion classique de la ligne droite, ont fait de vains efforts pour la déduire des propositions antérieures et sup-

(') Pour des renseignements détaillés, consulter : ENGEL et STäckEL, Theorie der Parallelinien onn Euklid bis auf Gauss (Ieipxig, Teubner, 1895 ), et R. Bonola, La Geometria non euclidea (Bologna,' 1906).

IRIS - LILLIAD - Université Lille 1 
primer cette prétendue solution de continuité qui, au dire de d'Alembert, fait le scandale de la Géomélrie ( $\left.{ }^{1}\right)$.

Nous trouvons dans Proclus ( $\left.{ }^{2}\right)$, le premier commentateur d'Euclide, des renseignements qui nous prouvent que le Postulatum était déjà un objet de discussion et de recherches chez les géomètres grecs des Écoles d'Alexandrie et d'Athènes. Proclus rapporte des essais de démonstration attribués à Posidonius, Geminus, Tolomeus; lui-même en ajoute un qui n'est pas plus heureux. Les mèmes préoccupations se fon $t$ jour plus tard cbez les Arabes Al-Narizi, Nasir-Eddin, chez les savants de la Renaissance Commandin, Clavius, Giordano Vitale, etc., qui, sous limpulsion du Commentaire de Proclus, recommencent à s'occuper de la question des parallèles. La plupart d'ailleurs prennent pour fondement le concept d'équidistance, soit en admettant l'existence de droites coplanaires équidistantes, soit en supposant que deux droites non équidistántes s'écartent d'un côté pour se rapprocher de l'autre, afin de prouver que la ligne équidistante d'une droite est une droite. Wallis $\left({ }^{3}\right)$, abandonnant les voies inutilement suivies par ses devanciers, cherche à résoudre la question d'une autre manière en admettant l'existence de figures semblables.

Saccheri ( $\left.{ }^{4}\right)$, Laubert $\left({ }^{5}\right)$, Taurinus $\left({ }^{6}\right)$ sont les premiers qui, tout cn demeurant convaincus que le célèbre postulat est vrai, et en tentant de le démontrer par des moyens quelquefois spécieux, ant la curiosité de rechercher ce qui advient quand on le met de côté. Ainsi ils obtiennent cer-

( ' $)$ 'a-l-on pas formulé gravement de nos jours cette opinion vraiment extraordinaire * que la morale elle-mème est intéressée à la démonstration du P'ostulatum d'Euclide »? Lire à ce sujet le curieux article de J. Axdrade : Euclidien et non euclidien (Ens. Math., r9oo).

$$
\begin{aligned}
& \text {.. On no stattendait guèso } \\
& \text { A voir la morale en celte afraire. }
\end{aligned}
$$

( $\left.{ }^{2}\right)$ Proclus, philosophe néo-platonicien, 412-485.

( $\left.{ }^{3}\right)$ Wallis, mathématicien anglais, 16 r6-1 zo3.

( $\left.{ }^{4}\right)$ Gerolamo Saccheri, S. I., 1667-1 733 .

(5) Lambert, I $7^{28-1777}$, né à Mulhouse, membre de l'Académie des Sciences de Berlin en $\mathbf{r}-63$.

( $\left.{ }^{6}\right)$ Taurinus, r $79^{\prime}-1874$. Son oncle, le jurisconsulte Scrweikart, dont les idées influèrent sans doute sur Ies siennes, avait été en correspondance avec GaUss.

IRIS - LILLIAD - Université Lille 1 
PRBMIÉRES IDÉES TOUCHANT LA GÉOMÉTRIE NON EUCLIDIENNE. 9

taines propositions caractéristiques de la Géomètrie génẻrale, prouvent par exemple que deux droites peurent être sécantes, parallèles ou non-sécantes, et que, dans ce dernier cas, elles ont une perpendiculaire commune à partir de laquelle elles divergent (Saccheri), ou encore que l'aire d'un triangle est proportionnelle a la différence entre la somme de ses angles et deux droits (Lambert). En même temps, Lambert et Taurinus, frappés de l'analogie qui existe entre Jes droites tracées sur un plan et les grands cercles tracés sur une sphère, entrevoient pourquoi une Géométrie basée sur le rejet du postulat euclidien ne doit a priori conduire à aucune contradiction Jogique.

- Par exemple, Taurinus, tout en écartant l'hypothèse dite de l'angle aigu parce qu'il en résulterait à ses yeux une conception de l'espace susceptible d'une infinité de déterminations, construit un système analytique adéquat à cette hypothèse, système qu'il nomme Géométrie logarithmicospherique, en substituant dans la formule de la Trigonométrie sphérique le rayon imaginaire $r \sqrt{-\mathrm{I}}$ au rayon réel $r$ de la sphère.

Les insuccès généraux ront commencer à éveiller chez les géomètres, et sous une forme suffisamment précise, cette idée nouvelle que le postulat euclidien doit être indémontrable, et qu'il faut l'admettre sans démonstration ou admettre quelque postulat équivalent. Dès $\mathrm{7} 79^{2}$, la question des parallèles était l'objet des recherches de Gauss ( ${ }^{1}$ ), et lorsque quelques années plus tard il réfutait une tentative faite par Wolfgang Bolyai ( ${ }^{2}$ ) (Theoria Parallelarum, r8o4) pour prouver l'existence de droites équidistantes, Gauss n'avait pas encore, semble-t-il, abandonné pour son propre compte l'espoir de vaincre la chimère. Mais la correspondance du grand savant avec Wachter, Schweikart, Schumacher (18161831), quelques Notices des Gelehrte Anzeigen de Goeltingue el quelques fragments épars dans ses papiers ne laissent

(1) Gauss, 1777-r855, professa lAstronomic à Gottingue.

(2) Wolfgang Bolyal Farkas, I775-1856, fut un professeur de Mathématiques de grand mérite. Voir la curieuse Notice que lui a consacrèe Adoly Dux, La tombe du savant (Pester Lloyd du 4 fév. $1880)$, Notice reproduite dans les Mémaires de la.Société des Sciences phys. el nat. de Bordeaux, t. V. 
aucun doute sur ce fait : qu'après ${ }^{813}$ il avait résolument coupé court à toute hésitation et conçu un projet d'exposition de la Géométrie non euclidienne, tout en demandant à ses amis, par excmple à Taurinus en 1824, , le silence sur ses communications dans la crainte qu'elles ne fussent pas comprises. Réserve admirable et regrettable tout à la fois!

\section{Les fondatenrs de la Géométrie non euclidienne : Lobats-} chewsky, Bolyai, Riemann. Leurs continuateurs. - Les premiers Mémoires spécialement écrits sur la nouvelle Géométrie indépendante du cinquième postulat d'Euclide sont dus à Nicolas Lobatschewsky $\left({ }^{1}\right)$ et à Jean Bolyai $\left({ }^{2}\right)$.

Dès 18 rŏ, Lobatschewsky s'occupe des parallèles et., à dater de 1825 , ses idées s'orientent nettement vers une Géométric basée sur la négation de la parallèle unique. En $\mathbf{1 8 2 6}$, il fait d'abord à Kazan une lecture publique sur l'Lxposition succincte des principes de la Géométrie; ensuite il publie les éléments et le développement de sa doctrine dans les Ouvrages suivants: Sur les fondements de la Géométrie, 1830; Géométrie imaginaire, 1837 ; Nouveaux fondements de Géométrie, $1838\left({ }^{3}\right)$; Recherches géométrigues sur la théorie des parallèles, $1840\left({ }^{4}\right) ;$ Pangéométrie, i 855, ce dernier Travail contenant l'exposé complet de son système. C'est pour rendre hommage au génie et à l'infatigable persévérance du savant russe, appelé à juste titre l'Euclide moderne, que le nom de Géométrie lobatschewskienne a été donné à l'ensemble de ses découvertes.

A la même époque, et sans connaître tout d'abord les travaux de Lobatschewsky, Jean Bolyai, encouragé par son père, publiait les résultats de ses recherches, en appendice au Tentamen de celui-ci, sous le titre Appendix scientiam spatii absolute veram exhibens, $1832\left({ }^{5}\right)$. Les propositions

( $\left.{ }^{1}\right)$ N.-I. Lobatschewsky, né à Nijni-Novgorod en r7g3, mort à Kazan en 1856 .

(2) J han Bolyal, savant hongrois, i 802-r860, fils de Wolviayg Bolyai.

( $\left.{ }^{3}\right)$ Traduction française par F. Matuedx. Bruxelles, Hayez, igor.

(4) Traduction française par J. Hoües (Mémoires de Bordeaux, 1866) réimprimée par Hermann. Paris, 1895 .

(5) Réimprimé en rgos par l'Académie des Sciences hougroise, traduit en italien par G. Batraglini (Giornale di Matematiche, 1868), en anglais par G. Bruge Halsted (Auscin, 1896). 
qui y sont mises en relief, et qui forment la science absolue de l'espace, sont démontrées par Iui indépendamment du postulat euclidien. Exemple, celle-ci : Les circonférences qui ont pour rayons les côtés d'un triangrle sont proportionnelles aux sinus des angles opposés. Bolyai termine son Appendix ( $\$ 43$ ) en construisant un cercle équivalent à un carré et en énonçant ce dilemme caractéristique : Ou l'axiome d'Luclide est vrai, ou la quadrature du cercle est possible.

D'après Euclide, la somme des angles d'un triangle quelconque est égale à deux droits; d'après Lobatschewsky, cette même somme est toujours inférieure à deux droits et variable; ces deux concepts sont également admissibles tant au point de vue logique qu'au point de vue pratique et, comme Gauss l'avait reconnu le premier, peuvent correspondre aussi bien l'un que l'autre à la Géométrie plıysique de notre univers. Il appartenait à Riemann ( ${ }^{1}$ ) de poser le premier jalon d'une voie nouvelle. Dans son Mémoire Sur les hypothèses qui servent de fondement à la Géométrie, lu en r85'́a à Ja Société philosophique de Gottingue, publié seulement en 1867 après la mort de l'auteur, et connu par une traduction française de Hoüel $\left({ }^{2}\right)$ en 1870 , on trouve cette remarque originale : * L'observation nous apprend avec une grande certitude que l'espace réel est non pas infini, mais illimité. "En d'autres termes, la distance de deux points de l'espace peut avoir une limite maxima; partant de là, il est loisible de créer un troisième système de Géométrie, analogue à la Géométrie sphérique, et où sans aucune contradiction l'on démontre que la somme des angles d'un triangle quelconque est supérieure à deux droits.

Dès lors, l'essor est donné; de nombreux savants, adeptes de la Géométrie non euclidienne, se lancent dans la voie ou-

(1) Beknhard Riemans, nė à Breselenz (llanovre) en i826, mort a Selasca (Italie) en r 866 . Il fut ( $\left.859^{-1} 866\right)$ un des successeurs de Gauss à la chaire de Gottingue.

( ${ }^{2}$ ) Nous ne pouvons oublier que c'est au savant professeur de la Faculté des Sciences de Bordeaux que nous devons les premières traductions des écrits de Bolyai, Lobatschewsky et Riemann. Hoüel, dont la puissance de travail était prodigieuse, n'avait pas hésité à apprebdre toutes les langues européennes dans le but de faire connatre à ses conten porains les cuures mathématiques les plus remarquables. 
verte par Lobatschewsky et Riemann, et leurs remarquables travaux éclairent d'un jour profond les origines et les idées fondamentales de la science de l'étendue. Pour ne parler que des plus célèbres, citons Bettrami ( $\left.{ }^{1}\right)$ et De Tilly ( $\left.{ }^{2}\right)$.

De même que la Géométrie plane non euclidienne de Riemann est analogue à la Géométrie sphérique, dans laquelle les grands cercles de ta sphère sont assimilès à des droites, de même Beltrami prouve en 1868 dans le Mémoire Saggio di interpretazione della Geometria non euclidea, publjé par le Giornale de Battaglini et traduit dans les Annales de l'École Normale de 1869 , que Ia planimétrie de Lobatschewsky est réalisée sur une surface particulière, la pseudosphère, dont les lignes géodésiques composent des figures à deux dimensions assimilables aux figures rectilignes. Cette découverte fut bientôt dépassée par les remarquables conceptions de De Tilly.

L'éminent savant belge, reprenant une idée de Cauchy, admet la notion de distance comme notion première irréductible, et prouve par des raisonnements irréfulables que les trois Géométries riemannienne, euclidienne, lobatschewskienne peuvent également, et sans aucune contradiction, en dériver tour à tour. [Voir Essai sur les principes fondamentaux de la Gémétrie et de la Mécanique (Mémoires de la Societé des Sciences physiques et naturelles de Bordeaux, 1880 ) et Essai de Géométrie analytique générale (Mémoires de l'Académie royale de Belgique, r 892)]. C'est donc à lui que l'on doit ce thèorème capital : il n'existe pas d'autre système de Géométrie possible que ceux de Riemann, Euclide, Lobatschewsky, et chacun est logiquement admissible indépendamment des deux autres. "Après ce qui précède $n$, dit De Tilly dans le paragraphe 51 de sa Géomélrie analytique que nous citons en entier, " est-il encore nécessaire de réfuter l'erreur des esprits attardés qui croient pouvoir trouver des démonstrations théoriques des principes expérimentaux de la Gëométrie ordinaire, et en particulier

( ${ }^{1}$ Ecgìne Beltrami, né à Crémone en $x 835$, mort à Rome le 18 février 1900 .

( $\left.{ }^{3}\right)$ Joseph-Marre DE Tilly, lieutenant général et membre de l'Académie royale de. Belgique, né à Y $\mathrm{Y}$ pres en $183_{7}$, mort à Schaerbeck le 4 -aout 2906 . 
du plus célèbre de tous, le principe de la parallèle unique, équivalent au Postulatum d'Euclide? Nous venons de voir que les systèmes de Géométrie théoriquement possibles sont en nombre infini, bien qu'ordinairement divisés en trois classes ou espèces; et que pour distinguer entre eux, ou même pour écarter un seul de ces systèmes, il a fallu invoquer l'expérience. Or, le principe de la parallèle unique n'est vrai que dans un de ces yroupes; dans un autre, il y a deux parallèles (ou une infinité, selon la manière de l'entendre); dans le troisième, le parallélisure est impossible.

"Démontrer ce principe sans invoquer l'expérience équivaudrait donc à démontrer l'impossibilité, même théorique, des systèmes où ce principe n'existe pas, c'est-à-dire à démontrer le contraire de ce qui a été établi dans le présent Mémoire.... Il y a donc lieu d'abandonner de pareilles tentatives et de reconnaître à la Géométrie usuelle son véritable caractère, qui est de constituer le plus simple des systèmes de Géométrie théoriquement possibles, en mème temps qu'elle s'accorde avec tous les résultats de l'expérience. "

- En fait, les démonstrations qu'on a essayé de donner du Postulatum sont de deux sortes, suivant qu'clles sont fondées ou non sur une propriété de l'infini. Dans le premier cas, l'infini géométrique étant impossible, toute preuve fondée sur lui est mauvaise; dans le second, il y a un défaut, pétition de principe ou cercle vicieux qui n'est pas loin, et il doit être facile de le meltre en évidence, les raisonnements auxquels nous faisons allusion ne consistant la plupart du temps qu'à admettre un postulat plus ou moins dissimulé et qui n'est pas davantage prouvé ( $\left.{ }^{1}\right)$.

(1) Parmi les innombrables tentatives, contentons-nous de citer celles de Bertand, de Genève, el de Cartos (Comptes rendus, 1867) qui sont célèbres. Yers la fin de sa vie, Lagravas présenta à l'Académie un Mémoire sur les parallèles; pendant qu'on en faisait la lecture, il interrompit et retira le manuscrit en disant : "Il faut que j'y songe encore" , 


\section{CHAPITRE II.}

LES DÉFINITIONS FT POSTLLATS D'APRÈ EUCLIDE.

LES TROIS GEOMÉTRIES.

4. Lès définitions. - Si le lecteur veut hien nous suivre sans parti pris, et étudier avec nous la question des origines de la Géométrie d'après Euclide même, il sera- peu à peu et facilement amené à se rendre compte de lexistence logique des trois systèmes euclidien, lobatschewskien, riemannien, en même temps qu'il acquerra la notion nette de leurs analogies et différences. Ouvrons donc les Éléments du grand géomètre grec, et analysons en premier lieu les définitions ct postulats du Livre $\mathbf{I}$, dans la grande edition de Peyrard (1816), avec texte grec, français et latin ( ${ }^{1}$ ).

Voici d'abord les définitions :

Point (déf. I). -- Le point est ce qui n'a pas de parties.

Ligne (déf. 2, 3). - Une ligne est une longueur sans largeur. Les extrémités de la ligne sont des points.

Droice (déf. 4). - La ligne droite est celle qui repose également sur tous ses points.

( $\left.{ }^{1}\right)$ Cette analyse a èté faite avec beaucoup de détails dans le second Volume des Lecons de Geométrie de Cisbscr-L-Lpdemans, puis d'une façon élémentaire, mais magistrale, dans les Premiers principes de Metageometrie de M. P. Maxsion, excellent opuscule peu connu en France et auquel nous ferons de fréquents emprunts. On la trouve encore dans de nombreux articles ou travaux dus à Cayliy, et à MM. Klein, Porncané, Flys-Sante-Marie, ctc., et, plus récemment, dans le Livre publié par M. D. Hiblent à l'occasion de l'inauguration du monument de Gauss à Goltingue: Fondements de la Géométrie. Voir enfin l'Ouvrage de M. Max Simon : Euclid und die sechs planimetrischen Bücher, Leipzig, Teubner, ıgor. 


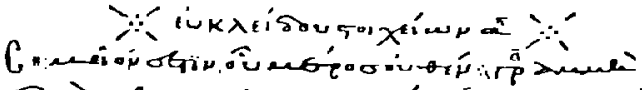

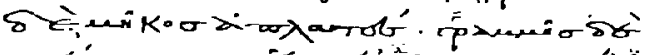

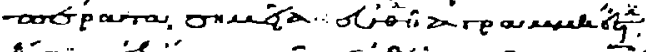

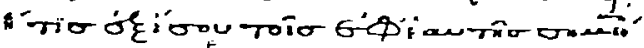

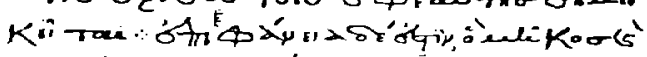

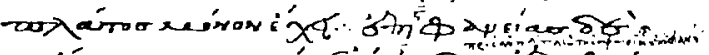

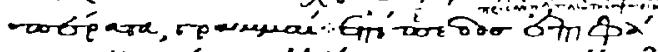

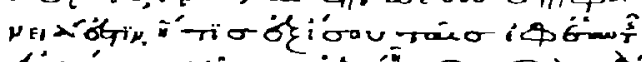

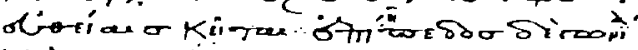

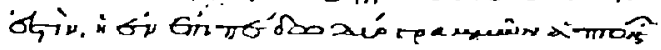

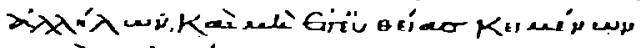

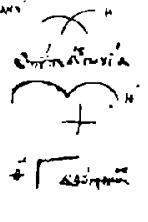

$+\sqrt{400}=$

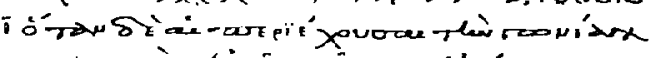

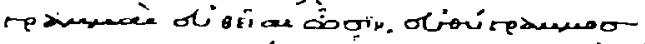
Kar

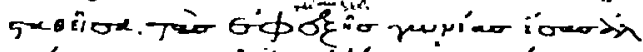

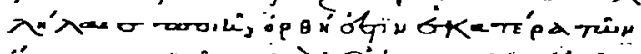

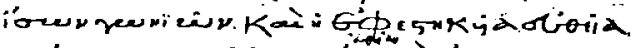
Kér тo K入入<smiles>CCCCCC</smiles>

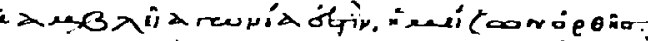

iि वैध

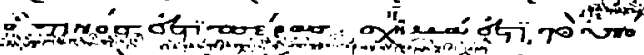

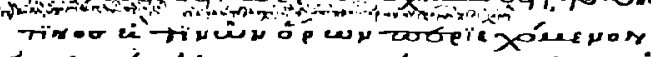

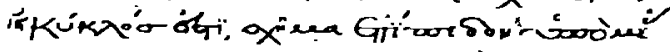

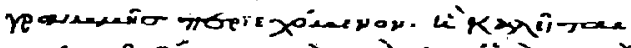

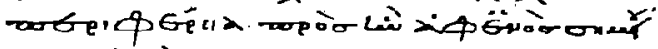

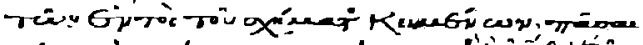

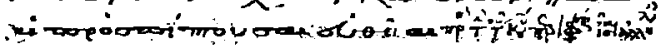

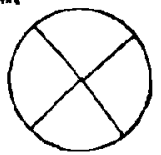

canimols

\section{Manuscrit des Éléments d'Euar.IDE.}

(Bibliothèque nationale de Paris, Fonds grec $n^{\circ}$ 2344, fol. 17 , XII ${ }^{2}$ siècle. Fac-similé du début : définitions du point, de la ligne, etc.) Gravure extraite de l'Histoire des Mathematiques, par J. BoYer. 
IRIS - LILLIAD - Université Lille 1 
Surface (déf. 5, 6). - Une surface est ce qui a seulement lonģueur et largeur. Les extrémités d'une surface sont des lignes.

Plan (déf. 7). - La surface plane est celle qui repose également sur toutes les droites qu'elle contient.

Angle (déf. 8, 9, I I, I 2). - Un angle rectiligne est l'inclinaison mutuelle de deux droites. Quand une droite en rencontre une autre en faisant avec celle-ci deux angles égaux de part et d'autre, chacun de ces angles s'appelle un angle droit, et la première droite est perpendiculaire à la seconde. L'angle obtus est celui qui est plus grand que l'angle droit; l'angle aigu est celui qui est plus petit que l'angle droit.

Cercle (déf. 15, 16). - Un cercle est une figure plane comprise par une seule ligne qu'on nomme la circonférence, toutes les droites menées à la circonférence d'un des points situés dans cette figure élant égales entre elles. Ce point se nomme le centre du cercle, ces droites se nomment les rayons du cercle.

Polygones, Triangles. - Les définitions (2o) à (33) du Livre I sont relatives a ux polygones, triangles, et à leurs différentes formes. Ajoutons-y ces deux définitions tirées du Livre XI :

Solide (déf. 12). - Un solide est ce qui a longueur, largeur et épaisseur. Les extrémités d'un solide sont des surfaces.

Sphère (déf. I禾, r7). - La sphère est une surface telle que toutes les droites menées d'un point appelé centre aux points de cetle surface sont égales entre elles; ces droites se nomment les rayons de la sphère.

J. Les postulats. - ${ }^{\circ}$ Qu'il soit demandé de mener de tout point à tout point une ligne droite.

$2^{\circ}$ Qu'il soit demandé de prolonger en ligne droite et en continuité une droite limitée.

$3^{\circ}$ Qu'il soit demandé de décrire un cercle de tout centre et de tout rayon.

Scientia, $\mathrm{n}^{\circ} 15$.

IRIS - LILLIAD - Université Lille 1 
$4^{\circ}$ Qu'il soit demandé que tous les angles droits soient égaux entre eux.

$5^{\circ}$ Qu'il soil demandé que si une droite rencontrant deux droites situées dans un mêrne plan fait d'un mème côté des angles intérieurs dont la somme soit moindre que deux droits, les deux droites prolongées indéfiniment se rencontrent du côté où la somme est inférieure à deux droits.

$6^{\circ}$ Qu'il soit demandé que deux droites ne contiennent pas d'espace.

6. Les définitions de la droite et du plan. - Les définitions qui précèdent et les quatre premiers postulats sont applicables à toutes les Géométries. Si les définitions de la droite et du plan ne nous paraissent pas au premier abord absolument claires, voici comment tous les géomètres sont néanmoins d'accord pour les entendre :

$I^{\circ}$ La ligne droite est la ligne entièrement définie par deux de ses points $A$ et $B$; et si l'on $y$ prend un troisième point quelconque $C$, les lignes droites définies par les couples de points $(A, B),(A, C),(B, C)$ sont identiques. Pour prolonger en ligne droite et en continuité une ligne droite limité $A B$, l'on peut concevoir de prendre sur cette droite, entre ses extrémités, deux points intermédiaires $C$ et $D$, et de faire glisser ensuite le système le long de ces deux points supposés fixes, comme une tringle supportée par deux clous, de façon que $A$ et $B$ se déplacent jusqu'en $A^{\prime}$ et $B^{\prime}$. Les droites définies par les couples de points $(A, B),(C, D),\left(A^{\prime}, B^{\prime}\right)$ sont identiques, donc $A B$ et $A^{\prime} B^{\prime}$ sont aussi des droites identiques, et cette dernière se nommera la droite $\mathrm{AB}$ prolongée jusqu'au point $\mathrm{B}^{\prime}$.

Ce prolongement en continuité peut se répéter autant que l'on veut; mais il est utile de remarquer que le gromètre grec, suivant l'habitude orientale, enseignait sans doute en plein air, et que peut-être même les figures dont il accompagnait ses démonstrations étaient simplement tracées sur le sable des jardins du Museum à Alexandrie; donc sa conception de l'espace, d'accord avec sa propre expérience, ne pouvait le porter à envisager que des êtres géométriques de dimensions finies. Pour lui comme pour nous, la droite est une ligne homogène entièrement déterminée par deux quelconques de 
PhOGRAMME DES PRINCIPALES PROPOSITIONS ÉLÉMENTAIRES. 17

ses points suffisamment rapprochés ( ${ }^{1}$ ); et ses extrémités, quelque reculées qu'elles soient, subsistent.

$2^{0}$ Le plan est la surface entièrement définia par deux droites déterminées et sécantes, $\mathrm{AB}, \mathrm{AC}$, qu'elle renferme, et si l'on y prend un point quelconque $D$, qui soit joint par la droite $\mathrm{DE}$ à l'un des points $\mathrm{E}$ de $\mathrm{AB}$, le plan défini par $\mathrm{AE}$ et ED est identique au plan de $\mathrm{AB}$ et $\Lambda \mathrm{C}$.

L'introduction de cette demière remarque permet alors d'expliquer pourquoi toute droite $M N$ qui a deux points $M$ et $\mathbf{N}$ dans le plan ( $A B, A C)$ y est renfermée tout entière. En effet, le plan ( $A B, A C$ ) est identique aux plans ( $A B, A M$ ) et $(\mathrm{AB}, \mathrm{AN})$; donc il renferme $\mathrm{AM}$ et $\mathrm{AN}$; mais le plan (AN, AN) étant entièrement défini par ces droites est identique à la fois aux plans ( $A M, M N$ ) et $(A B, A C)$, donc ce dernier renferme $\mathrm{MN}$.

Il n'est pas évident a priori qu'il existe une surface telle que, si I'on y prend deux points à volonté, la droite qui joint ces points soit tout entière sur cette surface. Aussi Bessel $a-t-i l$ pu critiquer justement cette définition du plan en disant qu'elle contient plus de conditions qu'il n'en faut pour le déterminer. Nous aurons l'occasion, au paragraphe 18, de revenir sur cel important sujet.

3o Le postulat 4 paraît superflu, mais en y regardant de près, on se convainc facilement que cette proposition était nécessaire pour éviter de considérer soit plusieurs sortes de plans, soit dans un même plan plusieurs sortes de droites. Son introduction, dit fort judicieusement M. Mansion, prouve combien Euclide avait profondément étudié les origines de la Géométrie. D'ailleurs le géomètre grec pouvait tout aussi simplement admettre ceci: Il n'y a qu'une seule espèce de droites.

7. Programme des principales propositions élémentaires de la Géométrio générale. - Cet ensemble de définitions et de postulats est complété par un postulat supplémentaire implicitement admis par tout le monde, celui de l'indéformabilité

(1) Jusqu'à nouvel ordre, nous laissons de coté, s'ils existent, les points exceptionnels, ou tels que par deux d'entre eux l'on pourrait mener plusieurs 2 oites.

IRIS - LILLIAD - Université Lille 1 
des figures en déplacement, indispensable pour l'égalité. Il suffit absolument, sous le bénéfice de la note restrictive du paragraphe précédent, pour prouver les vingt-six propositions élémentaires de la Géométrie générale qui comprennent: la théorie des angles adjacents et opposés, la construction du triangle équilatéral, les propriétés du triangle isoscèle, la détermination de la perpendiculaire à une droite menée par un point, les relations d'inégalité entre angles et côtés d'un mème triangle, et la démonstralion de la propriété de la droite d'être le plus court chemin entre deux de ses points. Il y a lieu d'ajouter à la liste une proposition d'usage intuitif, rentrant dans le cadre des notions communes, et nommée vulgairement postulat d'Archimède ( ${ }^{1}$ ).

On peut l'énoncer simplement ainsi : Si C est un point de la droite $A B$ situé entre $A$ et $B$, il y a un segment $A D$ de la droite, multiple de $A C$ et plus grand que AB. Ce postulat rend possible l'introduction de l'idée de continuité dans la Géométrie; mais, à dire vrai, ainsi que nous le verrons, il n'est pas indispensable.

8. Les hypothèses de Saccheri. - Arrivés à ce point, il nous devient facile d'aborder les considérations nouvelles d'où sortent indépendamment l'un de l'autre les trois systèmes de Géométrie, et de prouver qu'adopter librement tel ou tel d'entre eux revient à admettre en bloc, ou à rejeter séparément les postulats 5 el 6 .

Theorime de SAccheri. - Dans son Ouvrage ayant pour titre : Euclides ob omni novo vindicatus (Milano, i 73 ), ouvrage remis en lumière par Beltrami en 1889 , analysé successivement par M. Mansion, Annales de la Société scientifique de Bruxelles (I $89 \mathrm{I}$ ), puis par M. Giuseppe Veronese, Fundamentidi Geometria (Appendice historique, p. 569) ( ${ }^{2}$ ),

(') Voir Archimedis opera, texte de HEIBeng, i 880 , Vol. I, p. I .

(2) Sagcheri, dit M. Veronese, doit être considéré comme un véritable précurscur de Lobatschewsix et Rismans, quoique, victime des préjugés de son temps, suivant lesquels la seule Géométric possible était l'euclidienne, il se soit évertué a abattre de ses propres mains l'édıfice qu'il avait élevé, en démontrant la fausseté de ses deux nouvelles hypotheses. 
et traduit du latin en anglais par M. George Bruce Halsted, en allemand par M. Paul Stäckel dans sa Theorie der Parallelinien (Leipzig, 1895 ), Saccheri examine tour à tour trois hypolhèses correspondant aux trois cas que peut présenter un quadrilatere birectangle isoscèle $A C D B$ dans lequel les angles adjacents $A$ el $B$ sont droits, et les côtés $A C$ et $B D$ perpendiculaires sur AB sont égaux; dans cette figure I, la

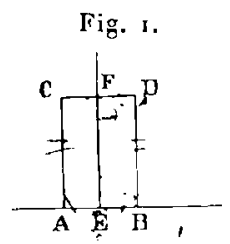

droite EF qui joint les milieux E et $\mathbf{F}$ de $A B$ et CD est aussi perpendiculaire à ces droites (médiatrice), et les angles Ciet D aux sommets sont égaux. Ils peuvent être droits, aigus ou obtus; Saccheri démontre nettement le premier que si l'hypothèse de l'angle droit, aigu ou obtus est réalisée dans un seul cas, elle est réalisée aussi dans tous les autres cas; et que selon que l'une ou l'autre de ces trois hypothèses serait admise pour vraie, la somme des angles d'un triangle serait respectivement égale, inférieure ou supérieure à deux angles droits. Il prouve ensuite facilement que l'hypothèse de l'angle obtus est incompatible avec le postulat 6 et s'attache particulièrement à celle de l'angle aigu, dans le but de démontrer sa fausseté, les raisons qu'il donne pour la rejeter étant d'ailleurs mauvaises ( ${ }^{1}$ ). Après Saccheri, Lambert a repris la même ètude, et tout en conjecturant que l'hypothèse de l'angle aigu pourrait être réalisée sur une surfiace qu'il ne définit pas et qu'il appelle sphère imaginaire, l'a rejetée également pour des motifs inacceptables ( ${ }^{2}$ ).

Il semble donc intéressant de suivre la route indiquée par ces deux esprits originaux pour en déduire avec toute rigueur

(1) Si elle était vraic, dit-il, deux droites pourraient avoir une perpendiculaire commune en un point commun situé à l'infini, ce qui rúpugne à la nature de la ligne droite.

( ${ }^{2}$ ) L'hypothèse de l'angle aigu entrafnerait l'existence d'une unité absolue pour les longueurs, ce qui semble a LAMBERT incompatible arcc notre façou de concevoir l'espace. 
les faits principaux qui se rapportent à chaque hypothèse. Nous nous conformerons à la Note du paragraphe 6, et nous considérerons d'abord une région du plan assez petite pour que deux points quelconques pris sur celte étendue déterminent une droite et une seule, tout point de cette droite appartenant au plan. Pour abréger, nous l'appellerons région normale ( $\left.{ }^{1}\right)$. Nous allons raisonner d'abord sur les figures tracées dans la région normale, puis nous indiquerons ce que deviennent les propositions démontrées quand on étend peu à peu les limites de cet espace.

9. Région normale. - Dans la région normale ( $\mathrm{H}$ ), tout triangle rectangle a un angle droit et deux angles aigus, tout quadrilatère birectangle est une figure convexe (conséquences des propositions XVI el XII du Livre I).

Trnonème. - Deux quadrilatères birectangles et isoscèles de (R) ont ensemble les angles aux sommets droits, aigus ou obtus.

Depuis Saccheri on a donné de ce théorème bien des démonstrations dans lesquelles, comme dans celle du géomètre italien, il est fait usage du postulat d'Archimède et du principe de continuité: Voici, par exemple, un raisonnement analogue à la méthode employée en Géométrie usuelle pour prouver que les aires de deux rectangles sont proportionnelles aux produits de leurs dimensions. On considère d'abord deux birectangles isoscèles qui ont la même base et des hauteurs différentes, puis deux birectangles isoscèles qui ont la mème hauteur avec des bases différentes; on compare enfin deux birectangles isoscèles quelconques de $(R)$ en les comparant isolément à un troisième birectangle formé avec la base de l'un et la hauteur de l'autre $\left(^{2}\right)$.

Mais le postulat d'Archimède n'est même pas nécessaire, ainsi que l'ont montré MM. Dehn $\left({ }^{3}\right)$ et Bonola $\left({ }^{4}\right)$. On peut

(1) Ou région de superposition d'après De Tilly.

${ }^{2}$ ) Voir la Note à la fin du volume.

( $\left.{ }^{3}\right)$ Voir l'article Die Legendre'schen Sätze über die Winkelsumme im Dreieck (Math. Annalen, t. LIII, p. 405-it39).

(') La Geometria non Fuclidea, p. 26-3r (Comptes rendus de t'Institut Lombard, rgo5). Voir égalemenb la Note, p. go. 
s'en passer pour exposer les fondements d'une Géométrie vraiment rationnelle (').

Corollaires. - Si les angles aux sommets d'un birectangle isoscèle sont droits, aigus, ou obtus, la somme angulaire de tout triangle rectangle, et aussi de tout triangle est égale, inférieure, ou supérieure ḋ deux droits. Par conséquent, si dans un seul triangle de ( $R$ ) la somme angulaire est égale, inférieure, ou supérieure à deux droits, dans tous les triangles de ( $\mathrm{R}$ ) la somme angulaire est aussi respectivement égale, inférieure, ou supérieure à deux droits.

10. Extension de la région normale. - Soient maintenant deux régions normales $\left(R_{1}\right),\left(R_{2}\right)$ ayant une partie commune (R). Toute propriété de ( $R$ ) appartient à $\left(R_{1}\right)$ et à $\left(R_{2}\right)$, donc elle appartient à leur somme $\left(R_{1}+R_{2}\right)$, si celle-ci continue à être une région normale; la proprieté appartient donc, sous le bénéfice de cette restriction, à une somme d'un nombre quelconque de régions normales $\left(R_{1}\right),\left(R_{2}\right), \ldots\left(H_{n}\right)$, juxtaposées. Mais un triangle quelconque peut toujours, alors même qu il ne saurait appartenil en entier à une région normale, se décomposer en autant de triangles qu'il sera récessaire pour que chacun de ceux-ci satisfasse à la condition imposée; le second corallaire du théorème précédent est donc vrai dans sa plus şrande généralité.

11. Hypothèse de l'angle droit. Géométrie euclidienne. Soicnt les droites $A B, C D$ faisant avec la sécante $A C$ les Fig. 2.

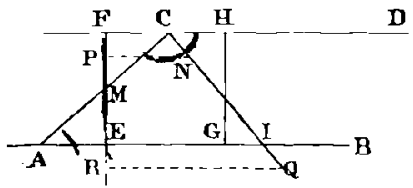

angles intérieurs d'un même còté BAC, DCA dont la somme égale deux droits ( $f g .2)$; la perpendiculaire AE abaissée du

(1) G. Bruce Halsten, Rational Geometry, p. 61.

IRIS - LILLIAD - Université Lille 1 
milieu M de AC. sur AB a pour prolongement MF également perpendiculaire sur CD, et toute perpendiculaire $H$ Ćr tirée à $\mathrm{AB}$ d'un point $\mathrm{H}$ de $\mathrm{CD}$ est unique, perpendiculaire sur $\mathrm{CD}$, et constamment égale à FE. Par un point donné $H$ hors de $A B$ on ne peut mener qu'un seul couple de lignes $H G$ et $C D$, et cette dernière ne saurait rencontrer $\mathrm{AB}$. Au contraire, toute ligne CI située dans l'angle ACD, c'est-à-dire de sorte que celte ligne et $\Lambda B$ fassent avec $\Lambda C$ d'un même côté des angles intérieurs de somme inférieure à deux droits, doit rencontrer $A B$; en effet, prenons sur CI la longueur quelconque CN, et tirons NP perpendiculaire sur EF. Si FP est supérieure ou au moins égale à $\frac{1}{n} \mathrm{FE}$, il suffit de preudre lo long de CI la ligne CQ égale à $n$. C., et d'abaisser QR perpendiculaire sur EF; FR est égale à $n$. FP, c'est-à-dire supérieure ou au moins égale à $F E$, par suite (Q est de l'autre côté de $\Lambda B$ par rapport à $C$, à moins qu'il ne soit sur $A B$. En tous cas, $C Q$ rencontre $A B$ en $I$, ce qui démontre le postulat 5 .

Enfin, il n'y a pas dans toute l'étendue du plan de points exceptionnels $A$ et $A^{\prime}$ par lesquels on pourrait faire passer deux droites distinctes $\Lambda \mathrm{BA}^{\prime}, \mathrm{ACA}^{\prime}$, car si cela était, en joiguant un point $B$ de la première à un point $C$ de la seconde, on formerait deux triangles $A B C, A^{\prime} B C$ pour la réunion desquels la somme angulaire vaudrait simultanément quatre droits, et quatre droits augmentés des angles $A$ et $A^{\prime}$. Le postulat 6 est donc vrai également.

1ý. Hypothèse de l'angle aigu. Géométrie lobatschewskienne. - Nous allons voir maintenant que l'hypothèse de l'angle aigu entraîne le rejet du postulat 5 et l'admission du postulat 6 .

Le second point résulte du raisonnement mème que nous venons de quitter, puisque dans les triangles $A B C, A^{\prime} B C$ réunis, la somme angulaire vaudrail simultanément moins et plus que quatre droits. Passons au premier; nous allons pour cela, suivant la méthode même adoptée par Lobatschewsky, étudier les positions que les diverses droites divergeant d'un point donné $O$ peuvent occuper par rapport à une droite donnée $x y$. 


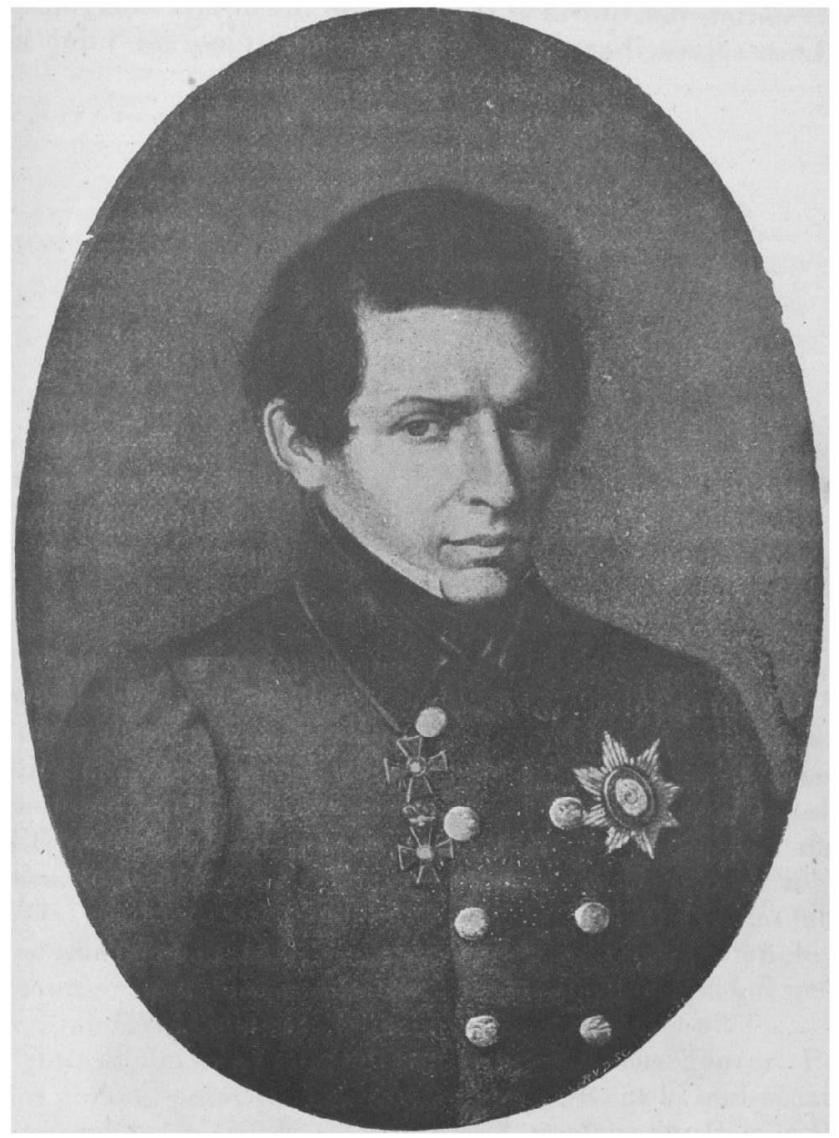

Portrait de Nicolas Lobatschewsky.

Gravure extraite de l'Histoire des Mathématiques, par J. BoYer.

IRIS - LILLIAD - Université Lille 1 
IRIS - LILLIAD - Université Lille 1 
Soient $(f g r .3)$ la perpendiculaire $O A$ et une oblique $O B$; du milieu $\mathrm{C}$ de $O B$, abaissons CD perpendiculaire sur $x y$, et prolongeons DC de CE égale à DC; la droite déterminée OEF qui joint les points $O, E$ est aussi perpendiculaire sur $D E$;

Fig. 3.

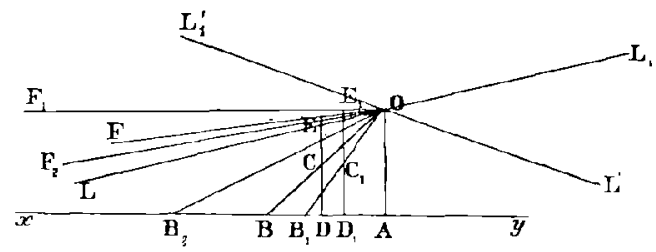

en un mot, à toute sécante OB on peut faire correspondre une droite $O F$ ayant avec $x y$ une perpendiculaire commune DE. Réciproquement, à tou te ligne du genre de OF, ou ayant avec $x y$ une perpendiculaire commune $E D$, on peut faire correspondre une sécante $O B$ et une seule, obtenue en joignant $\mathrm{O}$ a u milieu $\mathrm{C}$ de $\mathrm{ED}$, et prolongeant OC d'une longueur égale $\mathrm{CB}$. Voilà donc deux genres bien différents de droites passant par $\mathrm{O}$, et il n'y a plus qu'à les comparer.

Le premier genre contient autant de sécantes qu'il y a de points B sur $x y$, et toutes distinctes. Les droites du second genre sont également toutes distinctes, car deux d'entre elles $\mathrm{OEF}, \mathrm{OE}_{1} \mathrm{~F}_{1}$ ne pourraient se confondre sans donner naissance à un quadrilatère $\mathrm{DEE}_{1} \mathrm{D}_{1}$ ayant ses quatre angles droits. Enfin une droite du second genre ne peut se confondre avec une du premier sans donner naissance à un triangle où la somme angulaire dépasserait deux droits; le second genre est donc formẻ de non-sécantes en nombre indéfini.

Nous allons chercher ce qui sépare les deux genres. Prenons comme poịt de départ la sécante $O B_{1}$ et la non-sécante correspondante $\mathrm{OE}_{1} \mathrm{~F}_{1}$ de la figure 3 , portons sur $\mathrm{B}_{1} x$ la longueur $B_{1} B_{2}$ égale à $O B_{1}$, et traçons la non-sécante $O_{2} F_{2}$ correspondante de l'oblique $\mathrm{OB}_{2}$; de ce que la somme angulaire du triangle isoscèle $O B_{1} B_{2}$ est moindre que deux droits, on peut aisément conclure que les angles égaux $\mathrm{B}_{1} \mathrm{OB}_{2}$, $\mathrm{B}_{2} \mathrm{OF}_{2}$ sont inférieurs à la moitié de l'angle $\mathrm{B}_{1} \mathrm{OF}_{1}$, c'està-dire que $O F_{2}$ est dans l'angle $B_{2} O F_{1}$; donc nous écrirons 
les inégalités :

$$
\mathrm{B}_{2} \mathrm{OA}>\mathrm{B}_{1} \mathrm{OA}, \quad \mathrm{F}_{2} \mathrm{OA}<\mathrm{F}_{1} \mathrm{OA}, \quad \mathrm{B}_{2} \mathrm{OF}_{2}<\frac{\mathrm{OB}}{\mathbf{2}} .
$$

Appliquons la mêrne construction à partir de $\mathrm{OB}_{2}$, et nous en déduirons une oblique $O B_{3}$ et une non-sécante $O E_{3} F_{3}$ telles que nous pourrons écrire les nouvelles inégalités d'angles :

$$
\mathrm{B}_{3} \mathrm{OA}>\mathrm{B}_{2} \mathrm{OA}, \quad \mathrm{F}_{3} \mathrm{OA}<\mathrm{F}_{2} \mathrm{OA}, \quad \mathrm{B}_{3} \mathrm{OF}_{3}<\frac{\mathrm{OB}_{1} \mathrm{I}}{2^{2}} .
$$

En continuant de la sorte aussi longtemps que nous voudrons, nous aurons construit : $1^{\circ}$ des sécantes $O B_{1}, O B_{2}$, $\mathrm{OB}_{3}, \ldots, \mathrm{OB}_{n}$ faisant avec $\mathrm{OA}$ des angles aigus croissants; $2^{\circ}$ des non-sécantes $\mathrm{OF}_{1}, \mathrm{OF}_{2}, \mathrm{OF}_{3}, \ldots, \mathrm{OF}_{n}$ faisant arec $\mathrm{OA}$ des angles aigus décroissants. D'ailleur's, comme $\mathrm{OB}_{n}$ est la bissectrice de l'angle $B_{n-1} O F_{n}$, les angles de la seconde suite sont plus grands que ceux de la première, et eufin, comme $\mathrm{B}_{n} O \mathrm{~F}_{n}$ est inférieur à $\frac{O \mathrm{~B}_{1} \mathrm{~A}}{2^{n}-1}$, tout ceci se résume en disant que ces angles ont pour limite commune un angle aigu $\alpha$ indépendant de $\mathrm{OB}_{1}$. La droite déterminée $\mathrm{OL}$ qui fait avec $\mathrm{OA}$ l'angle LOA égal à $\alpha$ sert de limite commune aux sécantes et aux non-sécantes sans appartenir à un genre ni à l'autre; Lobatschewsky l'appelle parallèle à $x y$ et désigne sous le nom d'angle de parallélisme correspondant à OA l'angle limite $\alpha$. Il y a une seconde parallèle $O L^{\prime}$ symétrique de $O L$ par rapport à $\mathrm{OA}$, et les droites indéfinies $\mathrm{LOL}_{1}, \mathrm{~L}^{\prime} \mathrm{OL} \mathrm{L}_{1}^{\prime}$ forment quatre angles deux à deux opposés : parmi uux LOL' et $\mathrm{L}_{1} O \mathrm{~L}_{1}^{\prime}$ limitent la région des sécantes à $x y$, tandis que LOL $L_{1}^{\prime}$ et $\mathrm{L}^{\prime} O \mathrm{~L}_{1}$ constituent celle des non-sécantes. Ces résultats entraìnent le rejet du postulat 5 .

Lobatschewsky a bien pris soin de démontrer les propriétés les plus importantes de ses parallèles (voir Recherćhes géométriques sur la théorie des parallèles). En voici quelques-unes :

(I7). Une parallèle conserve le caractère de parallélisine en tous ses points. lèles.

(18). Deux droites sont toujours réciproquement paralIRIS - LILLIAD - Université Lille 1 
(2/4). Si l'on prolonge de plus en plus loin deux lignes parallèles dans le sens de leur parallélisme, elles s'approcheront de plus en plus l'une de l'autre. En fait, la distance d'un point de l'une à l'autre peut tomber au-dessous de tout intervalle donné si petit qu'il soit, et les parallèles de Lobatschewsky sont aussi asymptotes.

Au contraire, si deux droites forment un angle, ou ont une perperdiculaire commune, la distance de l'une à l'autre augmente au delà de toute grandeur donnée quand on s'éloigne soit du sommet, soit de la perpendiculaire.

(25). Deux droites parallèles à une troisième sont parallèles entre elles. Ajoutons-y, pour terminer, celle-ci :

Deux droites non sécantes et non parallèles ont une perpendiculaire commune à partir de laquelle elles divergent.

13. Hypothése de l'angle obtus. Géométrie riemannienne. Le postulat 5 est vrai, et le postulat 6 doit être rejeté.

Postulat 5. - Deux droites quelconques du plan sont sécantes. Prenons en effet les droites $\mathrm{AB}, \mathrm{CD}$, et d'un point quelconque $\mathrm{C}$ de la deuxième tirons CA perpendiculaire sur la première ( $f i g .4)$.

Fig. 4.

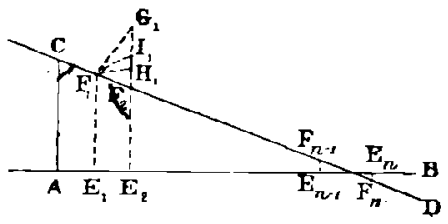

Si l'angle ACl) n'est pas droit, nous pouvons toujours le supposer aigu. Choisissons sur $C D$ des points $F_{1}, F_{2}, \ldots, F_{n}$ dont les projections $E_{1}, E_{2}, \ldots, E_{n}$ sur $A B$ déterminent sur $A B$, à partir de $\Lambda$, des segments égaux; les angles $\mathrm{CF}_{1} \mathrm{E}_{1}, \mathrm{CF}_{2} \mathrm{E}_{2}, \ldots$ sont tous obtus, et les projetantes $F_{1} E_{1}, F_{2} E_{2}, \ldots$ sont toutes décroissantes. Prenons sur $\mathrm{E}_{2} \mathrm{~F}_{2}$ les longueurs $E_{2} \mathrm{G}_{1}$ égale à $A G, E_{2} H_{1}$ égale à $E_{1} F_{1}$, et traçons $F_{1} G_{1}, F_{1} H_{1}$, puis la bissectrice $F_{1} I_{2}$ de l'angle $F_{2} F_{1} G_{1}$; la somme des angles $A C F_{2}$ et $\mathrm{E}_{2} \mathrm{~F}_{2} \mathrm{C}$ est supérieure a deux droits, et le second angle est inférieur au premier; par suite $F_{1} G_{1}$ est moindre que $F_{1} F_{2}$,

IRIS - LILLIAD - Université Lille 1 
et $I_{1} G_{1}$ est moindre que $I_{1} F_{2}$. Mais l'angle $H_{1} F_{1} F_{2}$ est visiblement plus grand que l'angle $G_{1} F_{1} H_{1}$, à cause des égalités

$$
\begin{aligned}
& H_{1} F_{1} F_{2}=H_{1} F_{1} E_{1}-F_{2} F_{1} E_{1}, \\
& G_{1} F_{1} I_{1}=2 \text { droits }-F_{2} F_{2} E_{1}-H_{1} F_{1} E_{1},
\end{aligned}
$$

donc $G_{1} H_{1}$ est inférieure à $H_{1} F_{2}$, et nous avons

$$
\mathrm{AC}-\mathrm{E}_{1} \mathrm{~F}_{1}<\mathrm{E}_{1} \mathrm{~F}_{1}-\mathrm{E}_{2} \mathrm{~F}_{2} \text {. }
$$

Désignons par $l$ la différence entre $A C$ et $E_{1} F_{1}$; nous pouvons poser :

$$
\begin{aligned}
& \mathrm{E}_{1} \mathrm{~F}_{1}=\mathrm{AC}-l, \\
& \mathrm{E}_{2} \mathrm{~F}_{2}<\mathrm{AC}-2 l,
\end{aligned}
$$

et en continuant de la sorte,

$$
\mathrm{E}_{n} \mathrm{~F}_{n}<\mathrm{AC}-n l .
$$

Soit $\lambda$ une longueur donnée aussi petite que nous voudrons, par exemple, inférieure ou au plus égale à $l$; $n$ étant choisi de sorte que $A C-(n-I) l$ soit plus petite que $\lambda$, la perpendiculaire $\mathrm{E}_{n} \mathrm{~F}_{n}$ ne peut plus rencontrer $C D$ au-dessus de $A B$, puisque sa longueur, moindre que $\lambda-l$, serait negative. Donc $\mathrm{AB}$ et $\mathrm{CD}$ se coupent entre $\mathrm{E}_{n-1}$ et $\mathrm{E}_{n}$, à moins que ce ne soit en l'un de ces deux points.

Rejet du postulat 6. - Deua droiles quelconques renferment un espace. D'après le raisonnement qui précède, deux droites $\Lambda \mathrm{B}$ et $\mathrm{CD}$ perpendiculaires à une troisième ont un point commun $\mathrm{O}$; et comme les propriétés de la congruence sont valides pour la totalité du plan, ces droites ont un second point commun $\mathrm{O}^{\prime}$; si de $\mathrm{O}$ comme centre, avec un rayon arbitraire, nous décrivons une circonférence les coupant en $a$ et $b$, et que nous partagions l'arc $a b$ en $n$ parties égales, les droites joignant $O$ à ces points de division viennent par symétrie, et quel que soit $n$, se couper de nouveau au point $O^{\prime}$; de plus, en prenant sur la circonférence des arcs successifs $b c, c d, \ldots$ égaux à $a b$, et leur appliquant la décomposition ci-dessus, il devient clajr que toutes les lignes du plan passant par $O$, rencontrant la circonfërence, se recoupent en $O^{\prime}$. Enfin, soient deux lignes quelconques MN, PQ; le postu- 


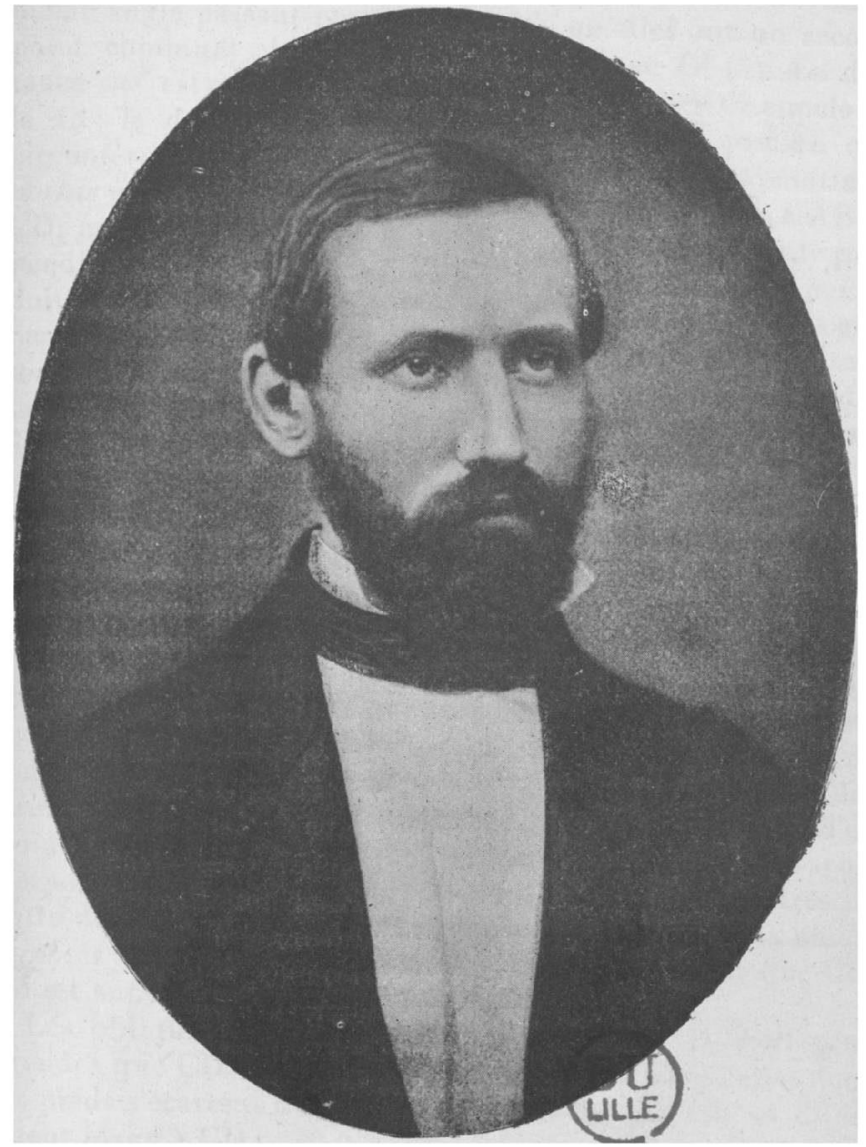

Portrait de Bernhard Riemann.

IRIS - LILLIAD - Université Lille 1 
IRIS - LILLIAD - Université Lille 1 
lat 5 étant vrai, elles ont au moins un point commun $\omega$; si on les transporte de façon à les appliquer sur deux droites de même angle passant par $O$, on vérifie qu'elles ont un second point commun $\omega^{\prime}$ qui coincide alors avec $\mathrm{O}^{\prime}\left({ }^{1}\right)$. La distance wis' est invariable et toujours égale à $O O^{\prime}$; appelonsla $2 \Delta$; la droite riemannienne est donc finie, comme une circonférence, et sa longueur totale vaut $4 \Delta ; \omega$ et $\omega^{\prime}$ sont dits points opposés. Si AC est perpendiculaire commune à $\mathrm{AB}$ et $\mathrm{CD}$, nous avons $\mathrm{OA}=\mathrm{O}^{\prime} \mathrm{A}=\mathrm{OC}=\mathrm{O}^{\prime} \mathrm{C}=\Delta$; or, toute perpendiculaire élevée sur $\mathrm{AC}$ en un quelconque $\mathrm{M}$ de ses points doit couper AC de nouveau en $\mathrm{M}^{\prime}$; comme $\mathbf{M M}^{\prime}=\mathbf{2} \Delta$, cette perpendiculaire passe aussi par $\mathrm{O}$ et $\mathrm{O}^{\prime}$, donc $\mathrm{OM}=\mathrm{O}^{\prime} \mathrm{M}=\Delta$; tous les points d'une droite riemannienne sont ainsi à la distance $\Delta$ de deux points opposés particuliers qu'il est permis d'appeler les centres de la droite.

Les extensions successives de la région normale ajoutent sans cesse de nouvelles étendues aux premières quand on se place dans les hypothèses euclidienne ou lobatsckewskienne, mais il n'en est plus de même en Géométrie riemannienne, où la somme $\left(R_{1}\right)+\left(R_{2}\right)+\ldots+\left(R_{n}\right)$ en vient, quand $n$ est suffisamment grand, à atteindre et dépasser toute l'étendue finie du plan; on voit de plus quelles sortes de restrictions doivent être apportées à ccrtaines propositions fondamentales du début; par exemple, deux points $A$ et $B$ ne définissent une droite que quand leur distance est différente de $2 \Delta$; d'un point $\mathrm{C}$ pris hors d'une droile $\mathrm{AB}$ on peut abaisser une seule perpendiculaire à $A B$ quand $C$ n'est pas un des centres de cette droite, et cette perpendiculaire coupe $A B$ en deux points opposés $\mathrm{D}, \mathrm{D}^{\prime}$ tels que CD est inférieure à $\Delta$ tandis que $\mathrm{CD}^{\prime}$ lui est supérieure.

Les obliques dont les pieds s'écartent de $\mathrm{D}$ sont plus grandes que $C D$ et vont en croissant jusqu'à $\mathrm{CD}^{\prime}$; celles dont les pieds s'écartent de $\mathrm{D}^{\prime}$ sont plus petites que $C \mathrm{CD}^{\prime}$ et diminuent jusqu'ì CD.

L'angle extérieur à un triangle est plus grand que l'un quelconque des angles intérieurs non adjacents seulement dans le cas où la médiane aboutissant au côté commun est

(') Cette démonstration est empruntée aux Premiers principes de Nétagéométrie, p. 23 ct suivantes.

Scientia, n॰15.

IRIS - LILLIAD - Université Lille 1 
inférieure à $\Delta$; mais si cette médiane vaut $\Delta$ ou la dépasse, l'angle extérieur égale l'angle intérieur, ou est plus petit que lui. Enfin, un triangle rectangle peut avoir deux angles aigus, un angle aigu et un obtus, ou deux angles obtus, suivant que les deux côtés de l'angle droit sont ensemble inférieurs à $\Delta$, l'un inférieur et l'autre supérieur, ou tous les deux supérieurs.

Nous croyons inutile de multiplier les exemples; le lecteur quelque peu familier avec la Géométrie pourra faire lui-même dans la série des propositions le départ entre celles qui sont vraies sans restriction, et les autres; il y sera aidé par la connaissance de ce qui se passe sur la sphère, où les figures formées d'arcs de grand cercle sont analogues, quoique non identiques aux figures rectilignes du plan riemannien.

14. Etude inverse. - De ce qui vient d'être démontré dans les trois paragraphes précédents, il est aisé de conclure par voie de réduction à l'absurde que l'admission des postulats 5 et 6 ensemble, ou le rejet, soit du postulat 5 , soit du postulat 6 entrainent tour à tour l'hypothèse de l'angle droit, aigu ou obtus. Mais on pourrait également arriver aux mêmes conclusions par un raisonnement direct, d'après Euclide, Legendre, Lobatschewsky et De Tilly. C'est ce que nous allons nous proposer de faire rapidement.

A. On admet le postulat 6 .

'ThÉnge (Luclide, I, 27). - Si deux draites AB, CD font a'ec une sécante $\mathrm{EF}$ des angles alternes internes égaux $\mathrm{AEF}, \mathrm{EFD}$, elles ne se rencontrent pus.

Premier theorkme de Legrnde. - Dans tout triangle rectiligne, la somme des angles ne peut surpasser deux droils.

Le célèbre auteur a donné deux démonstrations de cette proposition; l'une est reproduite dans la troisième édition de ses Éléments de Géométrie (Paris, Didot, I800, livre I, prop. 19); l'autre est donnée dans la douzième édition (1823) livre I, prop. 19 , et reproduite dans les Recherches géométriques de Lobatschewsky sous le même numéro ( $\left.{ }^{1}\right)$.

( $\left.{ }^{1}\right)$ Traduction de Hoüer, p. 7 .

IRIS - LILLIAD - Université Lille 1 
Detriême théoneme de Legendre. - Si dans un seul triangle la somme des angles est égale à deux droits, il en sera de méme pour tout autre triangle.

La troisième édition de l'ouvrage de Legendre contient la démonstration de ce second théorème, mais on en trouve une plus courte dans l'ouvrage de Lobatschewsky déjà cité, sous le numéro 20.

\section{B. On admet les postulats 5 et 6 .}

Tutontrme (Euclide, I, 29). - Si le cinquième postulat est vrai, deux droites qui ne se rencontrent pas font avec une transversale des angles intérieurs dont la somme est égale à deux angles droits.

Theorime (Euclide, I, 3 I-32). - Ayant prolongé en BD un cóté $\mathrm{AB}$ du triangle $\mathrm{ABC}$, l'angle extérieur $\mathrm{CBD}$ est égal à la somme des deux angles intérieurs opposés ACB, $\mathrm{BAC}$, et la somme des trois angles intérieurs du triangle est égale à deux droits.

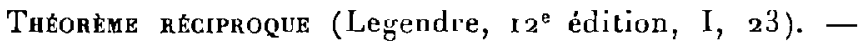
Réciproquement, si la somme des angles de tout triangle est égrale à deux droits, le cinquième postulat est vrai.

\section{On rejette le postulat 5 .}

Soient $x^{\prime} x$ une droite et $A B$ sa perpendiculaire abaissée de A. Il doit exister au moins une droite $y^{\prime} \mathrm{A} y$ telle que si l'angle $B A y$ est aigu, $A y$ ne rencontre pas $B x$; alors $A y^{\prime}$ ne rencontre pas non plus $B x^{\prime}$. En joignant $A$ à un point quelconque $\mathrm{C}$ de $\mathrm{B} x$, l'angle $\mathrm{BAC}$ est moindre que l'angle $\mathrm{BA} y$; donc CA prolongè ne peut couper de nouveau $x x^{\prime}$, et le postulat 6 est vrai pour tous les systèmes de droites tels que $x^{\prime} x$ et $\mathrm{AC}$. Il en résulte (d'après $\mathrm{A}$ ) que la somme des angles du triangle $\mathrm{ABC}$ est égale ou inférieure à deux droits. Or, si elle était égale à deux droits, Ay devrait rencontrer $\mathrm{B} x$, ce qui n'a pas lieu par hypothèse; cette somme est donc inférieure à deux droits, et la même propriété a lieu pour tous les triangles du plan (Lobatschewsky, Recherches géométriques, p. 20). 
D. On rejette le postulat 6 .

II faut donc supposer qu'il y a au moins un couple de droites $A B$, $C D$ renfermant un espace, et se coupant en deux points $O$ et $\mathrm{O}^{\prime}$. En raisonnant comme au paragraphe 13, il sera prouvé que loute droite passant par $O$ passe également par $\mathrm{O}^{\prime}$, que si deux droites $M N, P Q$ ont un point commun $\omega$, elles en ont un deuxième $\omega^{\prime}$ tel que $\omega \omega^{\prime}$ est invariable et égale à $\mathrm{OO}^{\prime}$, et que chacune est une ligne fermée.

Par suite, deux droites quelconques $M N, P Q$ se coupent en deux points; en effet, joignons le point $R$ de $M N$ au point $S$ de $P Q$; les droites $M N$ et $P Q$ ont chacune un second point commun $R^{\prime}$ et $S^{\prime}$ avec $R S$. Com d'ailleurs $R R^{\prime}$ égale $S^{\prime}$, et que $R S R^{\prime} S^{\prime} R$ est une ligne fermée, MN rencontre nécessairement $P Q$.

Theonìme. - Dans un triangle $\mathrm{ABC}$, la somme des angles est supérieure à deux troits.

Nous donnons la démonstration peu connue de De Tilly ( $\left.{ }^{1}\right)$. Soit ( $f g .5$ ) E le milieu de CB; menons AE et prolongeons

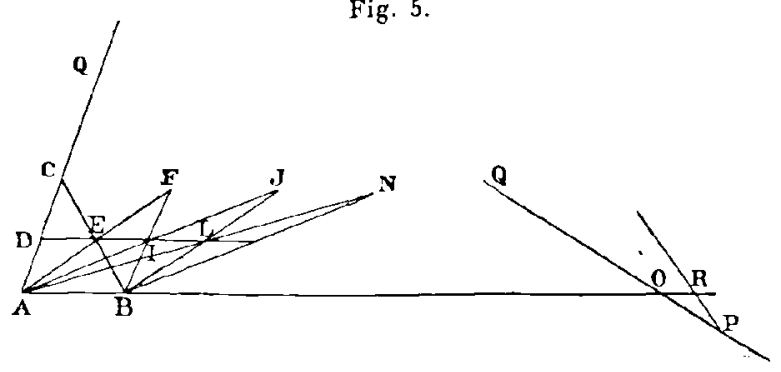

celte ligne d'une longueur EF égale à AE; menons aussi FB. Le triangle EFB sera égal à EAC. Supposons F à l'intérieur du triangle $\mathrm{CBO}, \mathrm{O}$ étant le point de rencontre de $\mathrm{ACQ}$ et $A B$, autre que $A$. Soit encore I le milieu de BF; menons AI et prolongeons cette ligne de la longueur égale IJ. Si le point $\mathrm{J}$ est à l'intérieur du triangle $\mathrm{BFO}$, nous prendrons le milieu $\mathrm{L}$ de $\mathrm{BJ}$, nous tracerons $\mathbf{A} \mathrm{L}$ et la prolongerons de la

(') Voir Mavsion, Mélagéométrie, p. 27.

IRIS - LILLIAD - Université Lille 1 
longueur égale LN; puis, si le point $\mathrm{Y}$ est à l'intérieur du triangle $\mathrm{BJO}$, nous ferons une construction analogue sur le triangle $A B . Y$, et nous continuerons ainsi indéfiniment.

Or, les triangles successifs ABC, ABF, ABJ, ABN, etc., ont toujours évidemment la mème somme angulaire; je dis que l'un des angles $A B J, A B N$, etc., finit par atteindre ou dépasser deux droits. En effet, soit D le milieu de AC; tirons les droites DE, EI, IL, etc.; les triangles DCE et EIB étant égaux, EI est le prolongement de DE, et ces longueurs sont égales; donc DFIL... est une ligne droite composée de segments égaux, qui rencontre $\mathrm{ADCQ}$ en un second point $\mathrm{P}$ situé au delà de $\mathrm{O}$, à la distance $\mathrm{OP}=\mathrm{AD}$, ainsi que $\mathrm{AB}$ en $R$; et si le nombre de ces. segments est suffisamment grand, l'extrémité $M$ du dernier finit par se trouver soit en R, soit sur RP ou son prolongement; l'angle ABM correspondant étant égal ou supérieur à deux droits, la proposition est démontrée.

Les conséquences des raisonnements précédents sont immédiates.

Le rejet du postulat 6 entraîne l'admission du postulat 5 et la Géométrie riemannienne; les postulats 5 et 6 ne peuvent se rejeter ensemble, et l'un au moins est nécessaire pour constituer un système complet de Géométrie.

En rejetáut le postulat 5, on est donc obligé d'admettre le postulat 6 , et la somme des angles d'un triangle est moindre que deux droits, ce qui caractérise la Géométrie lobatschewskienne.

La vraie nature des postulats ressort clairement de cette étude; ce ne sont, à tout prendre, que des définitions; aucune d'elles n'est renfermée dans les définitions ou postulats antérieurs, et aucune d'elles n'est davantage la conséquence de l'autre. La définition (4) et les postulats $1,2,4$ d'Euclide caractérisent le geNRE Droite, sous sa forme générale décomposable en trois variétés incompatibles entre elles: l'adjouction du postulat 5 seul distingue la variété ricmannienne, l'adjonction du postulat 6 seul distingue la variété lobatschewskienne, enfin la variété euclidienne s'obtient par l'adjonction des postulats jo et 6 réunis ( ${ }^{1}$ ).

(1) Mansion, Principes de Métagéométrie, p. $2 \mathrm{~g}$.

IRIS - LILLIAD - Université Lille 1 
On peut sans doute regretter que le géomètre grec n'ait pas donné plus de clarté à ses définitions de la droite et du plan; mais on est forcé de reconnaitre que, ces définitions admises, il ne pouvait choisir ses postulats avec une plus admirable sagacilé. Résumons-nous : à la porte de la Géométrie, trois routes d'égale importance et sans fusion possible s'offient à nous ( ${ }^{1}$ ); nous pouvons les parcourir indislinctement et aussi loin que nous voudrons sans autre obstacle que celui qui résulte de la plus ou moins grande difficulté analytique. Si nous choisissons la route euclidienne, c'est de plein gré et uniquement parce qu'elle est plus accessible que les deux autres; et suivant la remarquable expression employée par M. Poincaré dans l'article qui a pour titre : Sur les Géométries non euclidiennes (Revue générale des Sciences, I89I, p. 769), “ il n'y a pas de Géométries plus ou moins vraies; il y a seulement des Géométries plus ou moins commodes $n$.

15. Le plan elliptique de Cayley-Klein. - D'après ce qui a été vu au $n^{\circ} 13$, le plan de Riemann présente tous les caractères d'une surface sphérique, y compris le caractère exceptionnel des points opposés; a u fond, ce plan est bien une surface sphérique, au sens riemannien du terme, et les propriétés de la congruence y sont vraies, non seulement pour une région normale, mais pour la totalité de la surface.

Qu'arriverait-il si, dans l'hypothèse de l'angle obtus, on refusait au plan entier le postulat de congruence valide pour toute région normale, et sil'on admettait sans aucune exception le postulat de détermination de la droite par deux points? Cayley et Klein ont répondu à la question en faisant connaître les propriétés essentielles du plan elliptique, sur lequel la ligne droite est encore fermée et a une longucur finie, mais deux droites ne se rencontrent qu'en un seul point. La différence entre le plan sphère et le plan elliptique est fondamentale : le premier est une surface bilatère, le second une surface unilatère.

( $\left.{ }^{1}\right)$ Ds TrLy a ingénieusement symbolisé cette trifurcation par un schéma (Essai sur les Principes, p. $7^{3}$ ).

IRIS - LILLIAD - Université Lille 1 
Les surfaces ordinaires que nous sommes habitués à considérer sont bilatères; elles ont deux faces, un endroit et un envers, et l'on ne peut passer d'un point $\mathrm{A}$ de l'endroit à un point $B$ de l'envers par un chemin continu qu'en percant la surface ou traversant une de ses frontières éventuelles, c'està-dire le contour fermé qui limite une étendue quelconque de cette surface. Si, pour plus de précision, l'endroit est coloré en blanc, l'envers coloré en rouge, tout chemin continu qui lie $\mathrm{A}$ à $\mathrm{B}$ doit comprendre nécessairement un parcours AC fait sur le blanc, suivi d'un parcours CB fait sur le rougc. Deux triangles qui ont les côtés respectifs égaux sont congruents ou symétriques; dans le premier cas, ils ont même couleur, et on pent les superposer par simple glissement sur la surface; dans le deuxième, ils sont de couleurs différentes, et la superposition par glissement est impossible.

Il en va tout autrement avec une surface unilatère. Celleci n'a qu'une face et par conséquent point d'envers; une même couleur la recouvre partout entièrement, et l'on peut $y$ joindre deux points quelconques $A$ et $B$ par un parcours continu sans percer la surface ni en traverser le contour. Deux triangles qui ont les côtés respectifs égaux sont toujours congruents, ou, par simple glissement, un triangle peut ètre superposé à son symétrique. Möbius a fait connaitre, comme l'on sait, un moyen simple de construire un modèle de telle surface. Prenons, par exemple, un ruban de papier rectangulaire $A B C D$ : $D C$ recollé suivant $A B$ donne un cylindre, fragment de surface bilatère; mais, en le recollant suivant BA, on obtient une surface nouvelle où l'on peut reconnaître aisément les caractéristiques des surfaces unilatères citées plus haut.

16. Les Géométries non archimédiennes. - Conmme nous l'avons fait ressortir au $n^{0} 7$, le postulat d'Archimède est implicitement contenu dans toutes Ies explications qui précèdent. Fst-il absolument nécessaire? Pour caractériser son rôle, il fallait rechercher si, en le mettant de côté, des systèmes de Géométrie logiquement cohérents peuvent encore être construits; c'est ce qu'a fait, sous l'inspiration de 
34 LES DÉFINITIONS ET POSTULATS D'APHÈS EUCLIDE.

Hilbert, un de ses élèves. M. Dehn ( ${ }^{1}$ ) a reconnu qu'en donnant une certaine extension à l'idée de nombre :

I $^{\circ}$ On peut former une Géométrie où la somme des angles d'un triangle égale deux droits et où par un point passent une infinitè de non-sécantes à une droite donnée (Géométrie semi-euclidienne);

$2^{\circ}$ On peut également former une Géométrie compatible avec l'hypothèse de l'angle obtus et dans laquelle la droite a le caractère d'une ligne infinie et ouverte (Géométrie non legendrienne).

(') Die Legendre'schen Sätze über die Winkelsumme im Dreieck (Math. Annalen, t. LIII, p. 405-439). 


\section{CHAPITRE III.}

LA DISTANCE CONME YOTION fONDAMENTALE.

17. Les travaux de De Tilly. - Nous serons conduits aux mèmes conclusions que dans le Chapitre précédent si nous reprenons à un autre point de vue l'étude de la question qui nous a jusqu'ici occupés. La notion de distance peut être considérée par nous comme une des connaissances premières fondamentales que nous révèle l'expérience la plus simple, puisque nous y sommes naturellement conduits en comparant, au moyen d'un instrument étalon, les intervalles relatifs des points d'un solide reconnu comme invariable. Chaque intervalle entre deux points correspond de la sorte à un nombre, et nous disons que ce nombre est, par convention, Ia mesure de la distance des deux points.

$\mathrm{Or}$, il faut admettre que, dans tout système complet et rationnel de Géométrie, il doit exister entre les distances des couples de points de l'espace des relations générales de certaines formes; le nombre des intervalles figurant dans chacune de ces relations est en rapport avec le nombre de dimensions de l'espace que l'on étudie. Par exemple, dans l'espace à une dimension (ligne déterminée), il y a une et une seule relation générale entre les trois distances de trois points quelconques I, 2, 3 de cet espace. En effet, premièrement, il y en a au moins une, sans quoi, un des intervalles étant pris comme étalon, les deux autres seraient mesurés par rapport à lui par des nombres arbitraires, ce qui est inadmissible; en second lieu, il ne peut y en avoir plus d'une, car s'il $y$ en avait deux, un des intervalles pris comme étalon déterminerait entièrement les deux autres, ou en d'autres termes, les points 1 et 2 élant donnés, les intervalles $\mathrm{I}-3$ et 2-3 seraient constamment représentés par les mêmes nombres, quel que fùt le point 3 . Pareillement, dans l'espace à deux

IRIS - LILLIAD - Université Lille 1 
dimensions (surface déterminée), il y a une et une seule relation générale entre les six distances de quatre points quelconques, et dans l'espace à trois dimensions, le plus général que nous ayons à considérer ici, il y a une et une seule relation entre les dix distances de cinq points quelconques.

La découverte de cette relation a fait l'objet des recherches d'un certain nombre de savants. Lagrange, Mémoires de l'Académie des Sciences de Berlin, 1773 (Sur les P.yramides), et OEuvres complètes, Tome III, page 659, l'a fait connaître pour 5 points de l'espace euclidien. Cayley, The collected Mathematical papers, Tome I, art. I, puis Journal de Cambridge, Tome II, l'a transformèe par un determinant ( ${ }^{1}$ ).

M. Schering, Nachrichten de Gottingue, I 870, page 3 I I, et 1873 , pages 13 et 149 , a donné la relation analogue en Géométrie non euclidienne, en signalant son importance, mais sans y ajouter de développements. M. Mansion a montré, Annales de la Société scientifique de Bruxelles, 1895 ,

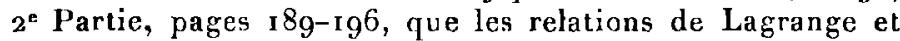
Schering pouvaient simplement s'établir au moyen des premiers principes de la Géométrie; mais ce sont surtout les Travaux de De Tilly qui ont donné la solution la plus complète et la plus générale de la question, et par suite, c'est au savant académicien belge que revient l'honneur d'avoir jeté le jour le plus profond sur les origines de la Géométrie, et assis d'une façon inébranlable les premiers principes de cette Science.

De Tilly a prouvé dans son Essai de Géométrie analylique générale que la relation des 5 points pouvait revêtir l'une des deux formes

$$
\left|\begin{array}{lllll}
\varphi(11) & \varphi(12) & \varphi(13) & \varphi(14) & \varphi(15) \\
\varphi(21) & \varphi(22) & \varphi(23) & \varphi(24) & \varphi(25) \\
\varphi(31) & \varphi(32) & \varphi(33) & \varphi(34) & \varphi(35) \\
\varphi(11) & \varphi(42) & \varphi(13) & \varphi(44) & \varphi(45) \\
\varphi(51) & \varphi(52) & \varphi(53) & \varphi(54) & \varphi(55)
\end{array}\right|=0
$$

(1) Consulter Rouché et oe Comberousse, Traité de Géomélrie, 6* et $7^{*}$ éditions, $7^{\circ}$ Partie, Nate I.

IRIS - LILLIAD - Université Lille 1 
olt

$$
\left|\begin{array}{lccccc}
0 & \text { I } & \text { I } & \text { I } & \text { I } & \text { I } \\
\text { I } & \varphi(11) & \varphi(\text { I }) & \varphi(13) & \varphi(14) & \varphi(15) \\
\text { I } & \varphi(21) & \varphi(22) & \varphi(23) & \varphi(24) & \varphi(25) \\
\text { I } & \varphi(31) & \varphi(32) & \varphi(33) & \varphi(34) & \varphi(35) \\
\text { I } & \varphi(41) & \varphi(42) & \varphi(43) & \varphi(14) & \varphi(45) \\
\text { I } & \varphi(5 I) & \varphi(52) & \varphi(53) & \varphi(54) & \varphi(55)
\end{array}\right|=0,
$$

o désignant une certaine fonction inconnue, mais telle que dans le premier cas on ait

$$
\varphi(\text { I })=\varphi(22)=\varphi(33)=\varphi(44)=\varphi(55)=1,
$$

et, dans le deuxième, ces mêmes symboles aient pour valeur commune zéro. D'ailleurs la seconde forme peut se déduire de la première par une hypothèse particulière et un passage à la limite.

Si l'on définit chacun des points du système par trois nombres ou coordonnées $x, y, z$, la fonction inconnue qui vérifie le déterminant I doit être, d'après De Tilly, telle que, $\varepsilon$ élant employé pour désigner à volonté $+\mathrm{I}$ ou $-\mathrm{I}$, l'on ait

$$
\text { (III) } \varphi(p q)=\frac{\mathrm{I}+\varepsilon\left(x_{p} x_{q}+y_{p} y_{q}+z_{p} z_{q}\right)}{\sqrt{1+\varepsilon\left(x_{p}^{2}+y_{p}^{2}+z_{p}^{2}\right)} \sqrt{1+\varepsilon\left(x_{q}^{2}+y_{q}^{2}+z_{q}^{2}\right)}} \cdot
$$

Dans cette égalité, les radicaux doivent être positifs. Quant à la fonction $\varphi$ qui vérifie le déterminant $I l$, elle doit être représentée par

$$
\varphi(p q)=\sqrt{\left(x_{p}-x_{q}\right)^{2}+\left(y_{p}-y_{q}\right)^{2}+\left(z_{p}-z_{q}\right)^{2}} .
$$

De Tilly a montré d'ailleurs que, s'il existait d'autres formes que I et II pour représenter un système de 5 points, leur découverte ne conduirait pas à un nouveau système de Géométrie distinct de ceux qui résultent des fonctions déjà trouvées (').

Il n'y a donc que trois systèmes de Géométrie possibles. Deux d'entre eux correspondent pour la forme III de? à $\varepsilon=\mathrm{I}$ et à $\varepsilon=-\mathrm{I}$; ce sont respectivement le système rie-

(') Essai de Gémélrie analytique générale, Note IV.

IRIS - LILLIAD - Université Lille 1 
mannien et le système lobatschewskien; le troisième. donné par la forme IV, est le système euclidien et l'on prouve que, moyennant une certaine hypothèse, il peut être considéré comme une limite commune des deux premiers.

18. La droite ot lo plan d'après Cauchy. - Leibniz donne cet énoncé géométrique basé sur l'idée de mouvement : La droite est la ligne telle que, si l'on immobilise deux de ses points, tous les autres sont immobilisés par cela seul. a Sit corpus aliquid, cujus duo puncta sint immota et fixa, ipsum autem corpus nihilominus movealur, tunc omnia puncta corporis quiescentia incident in rectam qua per duo puncla fixa transit " (OEuvres math. de Leibniz, èdition Gerhardt, t. V, p. 137 ).

Il en résulte qu'un point $C$ extérieur à la droite $A B$ des deux points fixes se meut, et que, ses distances à ces points étant supposées invariables, il y a au moins un autre point $D$ de l'espace dont les distances DA et DB à ces mêmes points sont égales à CA et CB.

Cette remarque a donné naissance à la définition plus avantageuse de Cauchy : La ligne droite $\mathrm{AB}$ est le lieu géométrique des points $\mathrm{M}$ tels qu'il n'y a aucun autre point $\mathrm{D}$ de l'espace pour lequel on ait $\mathrm{MA}=\mathrm{DA}$ et $\mathrm{MB}=\mathrm{DB}$. S'il est admis qu'il existe une relation générale entre les trois intervalles $M A, M B, \Lambda B$, l'on peut facilement en conclure que les droites $A B, M A$ et $M B$ sont identiques, ou, ce qui revient au même, que tout point $D$ extérieur à l'une est aussi extérieur aux deux autres.

Nous définirons le plan d'une manière semblable en disant avec Cauchy : Le plan ABC est la surface lieu des points $\mathrm{M}$ tels qu'il n'y a aucun autre point $\mathrm{D}$ de l'espace pour lequel on puisse avoir $\mathrm{MA}=\mathrm{DA}, \mathrm{MB}=\mathrm{DB}$ et $\mathrm{MC}=\mathrm{DC}$. S'il est admis qu'il $y$ a une relation générale entre les six intervalles $M A, M B, M C, A B, B C, A C$, l'on peut facilement en conclure que les plans $A B C, A B M, B C M$ et $A C M$ sont identiques, parce que tout point $D$ extérieur à l'un est aussi extérieur aux trois autres. Partant de là, nous savons vérifier ce théorème :

Toute droite MN qui a deux points $\mathrm{M}$ et $\mathrm{N}$ dans un plan $\mathrm{ABC}$ est contenue tout entière dans ce plan. 
Ceci résulte de ce que tout point $D$ extérieur au plan est extérieur à la droite $\mathbf{M N}$, et réciproquement.

En résumé, prenant la notion de distance comme la seule notion fondamentale de la Géométrie, nous avons pu retrouver sans exception tous les points de notre premier expose; donc tous les postulats admis sont bien compatibles entre eux, et l'existence de trois systemes de Géométrie indépendants est prouvé d'une façon inattaquable. 


\section{CHAPITRE IV.}

LA GÉOM ÉTRIE GÉNÉRaLE DANS LE PLAN ET DANS L'ESPACE.

19. La Géomètrie générale dans le plan. - Le lecteur quelque peu familier avec les connaissances géométriques qui font partie du programme de l'enseignement classique peut désormais, divisant les propositions de la Géométrie en deux groupes, distinguer d'un côté celles qui sont vraies sans exception dans tous les systèmes, de l'autre celles pour lesquelles l'énoncé doit subir une modification quelconque en passant d'un système à l'autre; et encore, parmi ces dernières, serait-il parfois possible d'adopter une formule s'appliquant à tous les cas, et qui fasse rentrer la proposition qui en est l'objet dans le groupe de la Géométrie générale $\left(^{1}\right)$.

En voici un exemple caractéristique. Dans la Gẻométrie d'Euclide, la somme de deux angles opposés d'un quadrilatère convexe $\mathrm{ABCD}$ inscrit dans une circonférence est constante et égale à deux angles droits. Cette proposition est fausse en Géométrie non euclidienne, puisque la somme de ces deux angles peut varier parfois dans des limites assez étendues. Or, cette différence provient de ce que l'énoncé euclidien est surabondant. Le sens de l'énoncé que nous venons de citer est celui-ci :

$$
\mathrm{A}+\mathrm{C}=\mathrm{B}+\mathrm{D}=\mathbf{2} \text { droits. }
$$

En Géométrie euclidienne, et dans cette Géométrie seule, la scconde égalité est la conséquence inévitable de la première qui est toujours vraie.

(1) Con'sulter, par excmple, G. Bruge HaLsted, Rational Geometry (New-York, Wiley, 1904), et Dassen, Tratado elemental de Geometria (Buenos-Ayres, Coni, r9o4).

IRIS - LILLIAD - Université Lille 1 
La formule de la Géométrie générale sera donc :

Dans tout quadrilatère convexe inscrit dans une circonférence, la somme de deux angles opposés égale celle des deux autres.

Cette formule est d'autant meilleure que tout esprit qui réfléchit sera instinctivement amené à la rapprocher de la suivante, qui est également générale :

Dans tout quadrilatère convexe circonscrit à une circonférence, la somme de deux cótés opposés égale celle des deux autres.

En comparant les deux énoncés, l'élève intelligent soupconnera, dès ses premiers pas dans la Gréométrie, l'existence d'un principe général de corrélation dont par la suite les manifestations lui seront fréquentes.

En généralisant les observations qui précèdent, on se convainc bientôt, pour peu que l'on y prête attention, qu'un assez grand nombre de théorèmes concernant les parallèles euclidiennes tiennent leur vérité, non de ce que ces lignes ne se rencontrent pas quoiqu'on les prolonge à volonté, mais uniquement de ce qu'elles ont une perpendiculaire commune de position particulière; s'il en est ainsi, ces théorémes doivent faire partie de la Géométrie générale. Pour ne citer qu'un exemple, prenons la proposition que voici :

Dans un triangle euclidien, la ligne $\mathrm{B}^{\prime} \mathrm{C}^{\prime}$ qui joint les milieux $\mathrm{B}^{\prime}$ et $\mathrm{C}^{\prime}$ des deux côtés $\mathrm{AB}$ el $\mathrm{AC}$ est parallèle au troisième cóté $\mathrm{BC}$.

Formulons-la de la façon suivante, qui, au point de vue euclidien, est entièrement équivalente :

Dans un triangle, la droite $\mathrm{B}^{\prime} \mathrm{C}^{\prime}$ qui joint les milieux $\mathrm{B}^{\prime}$ et $\mathrm{C}^{\prime}$ des deux cótés $\mathrm{AB}$ et $\mathrm{AC}$ st perpendiculaire sur la médiatrice du troisième côté $\mathrm{BL}$.

Nous l'avons de la sor ue remplacée par une proposition de Géométrie générale facile à démontrer. Pour cela, abaissons $\mathrm{AD}, \mathrm{BE}, \mathrm{CF}$ perpendiculaires sur $\mathrm{B}^{\prime} \mathrm{C}^{\prime}(f g .6)$. Ces trois lignes sont égales par suite des égalités de triangles $\mathrm{ADC}^{\prime}$ et $\mathrm{BEC}^{\prime}, \mathrm{ADB}^{\prime}$ et $\mathrm{CFB}^{\prime}$; mais dans le quadrilatère birec- 
42 La géonétrie générale dans le plan et dans l'espace.

tangle et isoscèle BECF, la médiatrice GA' de EF est aussi celle de $B C$, donc, etc.

Fig. 6.

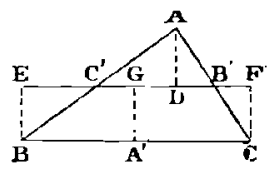

La réciproque a lieu également; concluons-en que :

Si dans un triangle deux huuteurs se coupent, la troisième passe par leur point de rencontre, et les trois lignes sont d'une façon générale. les bissectrices internes des angles du triangle qui a leur's pieds pour sommets $\left(^{1}\right)$.

Au Livre II, une notable partie des traités classiques de Géométrie appartient de même à la Géométrie non euclidienne, en particulier celle qui traite des relations de position entre la droite et la circonférence, ou entre deux circonfirrences, des arcs, cordes et tangentes, et de la mesure des angles au centre. Mais il ne saurait être question de la mesure des angles inscrits, puisque leur théorie repose sur le postulat 5. Et même, il faut remarquer que si toutes les constructions indiquées demeurent possibles et exécutables en Géométrie riemannienne, puisque deux droites y sont toujours sécantes, il n'en est plus nécessairenuent ainsi sur le plan lobatschewskien. Le problème bien connu : Décrire une circonférence par trois points donnés $\mathrm{A}, \mathrm{B}, \mathrm{C}$ va nous en fournir un exemple.

Le centre de la circonférence cherchée, quand elle existe, est à la rencontre des trois médiatrices de $\mathrm{AB}, \mathrm{BC}$ et CA.

Quand deux d'entre elles se coupent, la troisième passe par leur point d'intersection $O$, et ce point est le centre cherché, d'ailleurs unique.

Quand deux d'entre elles sont parallèles, la troisième leur est aussi parallèle, et le point O n'existe plus. Or Lobatschewsky a démontré, $\$ 31$ de ses Recherches géométriques, qu'il existait alors une courbe particulière nommée par lui

(1) Bilatères et trilatères ( vathesis, rgo2, p. ${ }_{18}^{8-1} 9^{3}$ ).

IRIS - LILLIAD - Université Lille 1 
horicycle ou courbe limite, et passant par les trois sommets du triangle. Cette courbe jouit de la propriété que les médiatrices de toutes ses cordes sont parallèles entre elles, et l'on peut la considérer comme la limite vers laquelle tend une circonférence dont le centre s'éloigne au delà de toute distance. L'horicycle ne peut pas être tracé mécaniquement ( ${ }^{1}$ ), mais on peut en déterminer avec la règle et le compas autant de points que l'on voudra, comme nous le verrons dans le Chapitre suivant.

Enfin, supposons que deux médiatrices, celles des còtés $\mathrm{AB}, \mathrm{AC}$, soient non-sécantes et aient une normale commune $\mathrm{DE}(f g \cdot 7)$.

$$
\text { Fig. } 7 \text {. }
$$

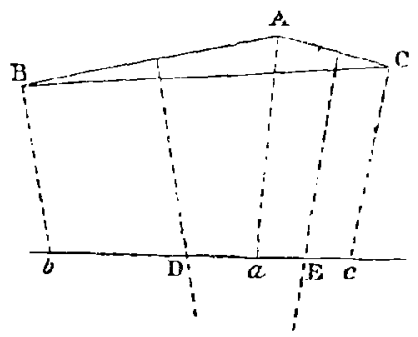

Soient menées A $a, \mathrm{~B} b, \mathrm{C} c$ perpendiculaires sur DE; comme elles ont même longueur, la médiatrice du côté BC est aussi perpendiculaire à $\mathrm{DE}$, et il existe une ligne déterminée passant par les trois points A, B, C. C'est le lieu géométrique des points pour lesquels la distance à la droite DE est constante et ègale à Aa. Cette ligne, qui reçoit le nom d'equidistante ou d'hypercycle, jouit de la propriété que les médiatrices de toutes ses cordes sont perpendiculaires à $\mathrm{DE}$, laquelle en est l'axe; s'il est évident que son tracé mécanique est très simple, il n'y a pas davantage de difficultè à en construire tous les points que l'on voudra, une fois que la droitc DE aura été elle-même dessinée.

L'horicycle et l'hypercycle ont toutes les propriétés de la circonférence, à condition de considérer les rayons du premier comme parallèles, et ceux du second comme normaux à

(') Ou du moins le mécanisme à employer pour cé tracé est relativement compliqué.

Scientia, $\mathrm{n}^{\circ} 15$.

IRIS - LILLIAD - Université Lille 1 


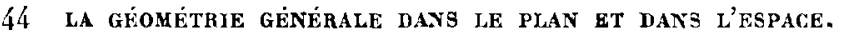

un même axe; ajoutons que toute circonférence riemannienne est aussi un hypercycle riemannien.

Voici encore une question capable de donner lieu à quelques observations intéressantes : Mener par un point donné A extérieur à une circonférence la tangente AT. La solution générale du problème est celle par la méthode dite du double rayon qui ne s'appuie que sur les propriétés du triangle isoscèle. Quand la circonférence est un horicycle ou un hypercycle, l'ón a à construire un quadrilatère trirectangle où deux côtés sont connus.

La Géométrie générale n'emprunte presque rien au troisième Livre usuel; c'est que celui-ci repose en entier sur la similitude des figures, et que dans les hypothèses non euclidiennes, comme Gauss l'avait le premier remarqué, et comme un raisonnement élémentaire permet aisément de s'en convaincre, deux figures semblables ne peuvent qu'être égales. Il faut toutefois faire exception pour les polygones réguliers, dont la possibilité générale et les propriétés essentielles ne tirent point leur origine du cinquième postulat d'liuclide. La construction effective des polygones convexes dérivés du carré, du triangle équilatéral et du pentagone exige seulement parfois des tracés plus compliqués qu'en Géométrie euclidienne ( ${ }^{1}$ ).

Il existe également une théorie des axes radicaux, ainsi que des pôles et polaires conduisant à un grand nombre de propositions formulées exactement comme en Géométrie euclidienne; si le lecteur est désireux de les étudier de plus près, il pourra consulter utilement à ce sujet les Leçons de Géométrie de Clebsch-Lindemann, la théorie du rapport anharmonique donnée par M. F. Klein dans son Mémoire Leber die sogenannte nicht euklidische Geometrie (Math. $A n n .$, I 87 I), et enfin la thèse inaugurale de M. Gérard, $S u$, la Géométrie non euclidienne, 1892.

20. La Géomètrie générale dans l'espace $\left({ }^{2}\right)$. - L'enseignement actuel divise le Livre $\mathrm{V}$ en quatre parties disposées à peu

( $\left.{ }^{1}\right)$ Consulter : Polygones réguliers sphériques et non euclidiens, (Le Matematiclie, 1902, p. 137-145).

(2) Le cinquième Livre de la Métagéométrie (Mathesis, rgor, p. $177^{-190) \text {. }}$

IRIS - LILLIAD - Université Lille 1 
près dans l'ordre suivant par beaucoup d'auteurs : Relations de position de la droite et du plan, plans et droites perpendiculaires. - Plans et droites parallèles. - Angles dièdres et dièdres droits. - Angles trièdres et polyèdres.

Conservons cette division usuelle. Si nous observons que dans l'espace riemannien toute perpendiculaire à un plan passe par deux points opposés dont la distance aux différents points de ce plan égale $\Delta$, et perce également le plan en deux points opposés, que de plus toute droite rencontre un plan en deux points également opposés, nous pourrons dire qu'à cela près, et d'une façon presque absolue, les propositions de la première el des deux dernières parties étant indépendantes du Postulatum d'Euclide appartiennent à la Géométrie générale; dans les unes, l'énoncé et la démonstration subsistent sans modifications, tel le théorème dit des trois perpendiculaires, avec ses réciproques et ses applications ordinaires; dans d'autres, si l'énoncé demeure, il y a lieu de présenter le raisonnement sous une forme qui puisse s'appliquer à tous les cas.

C'est ainsi que l'égalité de deux angles rectiligues d'un dièdre doit résulter uniquement de celle de deux triangles qui ont leur's trois côtés égaux chacun à chacun. Ainsi encore, c'est bien à tort, selon nous, que dans beaucoup d'Ouvrages de Géométrie, même parmi les meilleurs, le théorème sur la somme des faces d'un angle trièdre ou polyèdre est prouvé avec le secoursja cinquième postulat, dont il est tout à fait indépendant; quand on a prouvé que dans un trièdre une face est plus petite que la somme des deux autres, il suffit de prolonger une seule arête au delà du sommet pour en déduire que la somme des trois faces est moindre que quatre droits; s'il s'agit maintenant d'un angle polyèdre convexe de $n$ faces, il n'y a qu'à admettre la proposition pour un angle de $n-\mathbf{I}$ faces, et la généraliser pour une face de plus.

Enfin, pour certains théorèmes, la théorie des parallèles semble jouer un rôle qu'en réalité elle ne joue pas du tout, et, si l'on veut bien pénétrer un peu plus avant dans leur esprit, on ne tarde pas à s'en convaincre. En' voici un exemple bien remarquable:

Pour qu'un angle droit se projette sur un plan suipant un angle droit, il faut et il suffit, disent les Traités, qu'un 
côté au moins de l'angle soit parallèle au plan de projection.

La traduction non euclidienne de cette formule est la suivante:

Pour qu'un angle droit se projette sur un plan suivant un angle droit, il faut et il suffit que la projetante du sơmmet soit normale commune à un côté de l'angle au moins, et au plan.

Quant au texte même de la démonstration, il n'y a pour ainsi dire rien à changer.

A la fareur des explications qui précèdent, on voit que les principes fondamentaux de toutes les Géométries descriptives sont les mêmes, et qu'un point, une droite, un plan sont toujours parfaitement représentés au moyen de leurs projections ou de leurs traces; ceci pourra être utilisé au besoin.

Il reste à nous occuper de la partie du Livre $V$ qui traite des droites et plans parallèles. Or, le mot euclidien de parallèles est employé indifféremment, comme cela a déjà été remarqué, pour désigner à la fois les droites et plans qui ne se rencontrent pas, comme ceux qui ont une perpendiculaire commune; tandis que la nécessité s impose en Géométrie non euclidienne de distinguer, le terme de parallèles ayant un sens différent.

21. Théorie des droites et plans qui ont une normale commune. - $\mathrm{I}^{\circ}$ Deux droites $\mathrm{AB}, \mathrm{CI}$ perpendiculaires à un plan P sont dans un mème plan $Q$, et normales à la ligne d'intersection AC des deux plans. - Réciproque el corollaires évidents.

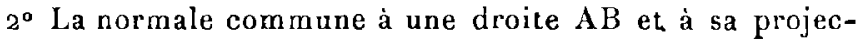
tion $a b$ sur un plan $P$ est aussi normale commune $\dot{a} \mathrm{AB}$ et au plan. - Réciproque.

$3^{\circ}$ Deux plans riemanniens quelconques et deux plans lobatschewskiens non sécants ont une normale communc renfermée dans tous leurs plans normaux communs.

$4^{\circ}$ Tous les plans riemanniens qui renferment une droite D ont une normale commune $\mathrm{D}^{\prime}$, et $\mathrm{D}$ est aussi normale commune à tous les plans passant par $\mathrm{D}^{\prime}$. Toute droite joignant $\mathrm{D}$ à $\mathrm{I}^{\prime}$ leur est normale commune, et vaut $\Delta$. I) et $\mathrm{D}^{\prime}$ sont réciproques. 
$5^{\circ}$ Deux droites quelconques riemanniennes $\mathrm{D}$ et $\mathrm{D}^{\prime}$ ont généralement deux perpendiculaires communes réciproques qui servent à mesurer, l'une $\mathbf{A A}^{\prime}$ la distance minima, l'autre $\mathrm{BB}^{\prime}$ la distance maxima de leurs points, et, dans ce cas, la distance $M^{\prime}$ de deux points quelconques de $\mathrm{D}$ et $\mathrm{D}^{\prime}$ oscille entre $\mathrm{AA}^{\prime}$ et $\mathrm{BB}^{\prime}$. $\mathrm{Or}$, il peut arriver que pour certaines positions relatives de $D$ et $D^{\prime}$ on ait $A^{\prime} A^{\prime}=B^{\prime} B^{\prime}$; alors toute ligne perpendiculaire a $\mathrm{D}$ et coupant $\mathrm{D}^{\prime}$ est aussi perpendiculaire à cette dernière, et sa longueur est constante et égale à $\mathrm{AA}^{\prime}$; quand $\mathrm{D}$ et $\mathrm{D}^{\prime}$ sont de la sorte équidistantes, deux perpendiculaires communes quelconques déterminent un rectangle gauche où les diagonales sont égales et font avec les côtés des angles alternes-internes ègaux (1).

$6^{\circ}$ Deux droites quelconques lobatschewskiennes ont une seule perpendiculaire commune. Sa construction peut se ramener à celle des points de rencontre d'une droite avec un hypercycle.

22. Thëorie des droites et plans paralléles. $-1^{\circ}$ Toute ligne $D$ parallèle à une ligne $D^{\prime}$ d'un plan $P$ est parallèle à sa projection $d$ sur ce plan, ainsi qu'à toutes les lignes du plan parallèles à $\mathrm{D}^{\prime}$ (y compris $d$ ). - Réciproques. - La droite $D$ ainsi définie est parallèle au plan.

$2^{\circ}$ Par un point $S$ on peut mener une infinité de lignes $D$ parallèles à un plan $P$, et appartenant à un cône de révolution qui a pour axe la normale $S s$ au plan.

$3^{\circ}$ Par une droite $D$ on peut mener deux plans $P$ et $P^{\prime}$ parallèles à une droite $D^{\prime}$. 'T'ous les plans $Q$ qui passent par $D$ peuvent alors se ranger en deux catégories : la première, renfermée dans le mêrne dièdre des plans $P, P^{\prime}$ que la droite $\mathrm{D}^{\prime}$, est formée de tous les plans qui coupent $\mathrm{D}^{\prime}$; l'autre, appartenant au dièdre adjacent, est formée au contraire de plans qui, ne coupant pas $\mathrm{D}^{\prime}$, ont chacun avec elle une normale commune.

$4^{\circ}$ Pour que deux plans lobatschewskiens soient sécants, il faut et il suffit que par un point du premier on puisse mener dans celui-ci deux lignes sécantes parallèles au second;

(') Consulter : Clifford, Preliminary Sketch on Biquaternions ('roceedings of London Math. Society, 1873, p. 381-3y5).

IRIS - LILLIAD - Université Lille 1 


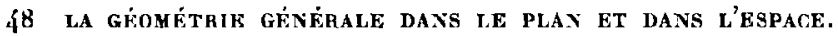

donc, si par deux droites parallèles on fait passer deux plans qui se coupent, l'intersection est parallèle aux premières droites.

50 Pour que deux plans lobatschewskiens $P$ et $Q$ soient parallèles, il faut et il suffit que par chaque point $A$ de $P$ on ne puisse tirer dans celui-ci qu'une seule ligne $A B$ parallèle à $Q$. Le plan qui projette $A B$ sur le plan $Q$ est alors normal commun à $\mathrm{P}$ et $\mathrm{Q}$, et réciproquement. Deux plans normaux communs à $P$ et $Q$ sont parallèles; ils forment avec ces deux plans une sorte d'angle polyèdre indéfini dont les quatre angles dièdres sont droits et qu'un plan diagonal coupe suivant deux trièdres où la somme des dièdres égale deux droits. La proposition précédente peut servir à vérifier simplement le théorème 28 des Recherches géométriques de Lobatschewsky : Lorsque trois plans se coupent deux à deux suivant des droites parallèles, la somme des trois angles dièdres ésale deux droits, théorème que le géomètre russe prouve assez péniblement par des triangles sphériques d'aire décroissante, et qui s'étend évidemment à un nombre quelconque de plans.

La Géométrie propre de la sphère et des figures tracées à sa surface est indépendante du postulat 5. Mais la mesure des aires et celle des volumes se font en Géométrie non euclidienne par des moyens différents; nous en reparlerons avec détails un peu plus loin.

Enfin, il est loisible de créer un Livre VIII non euclidien, et d'étudier les courbes dites usuclles, c'est-à-dire les lieux géométriques de points tels que la somme ou la différence de leurs distances à deux points fixes, à un point et à une droite, ou à deux droites soit constante. En Géométrie riemannienne, ces courbes sont toutes des ellipses; mais sur le plan lobatschewskien elles revêtent une grande variété de formes, ainsi que le montre la section du cône de révolution par un plan [Voir nos Études de Géométrie analytique non euclidienne, $\$ 18$ (Mémoires de L'Académie royale de Belgique, rgoo)]. 


\section{CHAPITRE V.}

LA TRIGONOMÉTRIE.

23. Formules des triangles. - Les explications renfermées dans les Chapitres précédents donnent la vision nette du champ qui apparient à chacun des trois systèmes de Géométrie; toutefois, si nous demeurons convaincus que chacun d'eux est logiquement possible au même titre que les autres, il peut nous rester un dernier doute qu'il importe essentiellemenl de lever : les raisonnements de la Géométrie euclidienne se font sur des figures que l'on sait toutes construire (et Euclide s'était imposé cette règle invariable, qu'on est souvent porté à négliger); ces raisonnements acquièrent par cela même une grande force de pénétration. En est-il ainsi dans les Géométries non euclidiennes, et sait-on également, par le seul secours de leurs postulats, construire à la règle et au compas les parallèles, les perpendiculaires communes, les équidistantes, les horicycles, les fractions de la distance maxima $2 \Delta$, ainsi que les figures stéréométriques qui en dépendent, etc.?

Lohatschewsky n'a rien dit à ce sujet. Bolyai est le premier qui ait donné une construction des parallèles; seulement il s'appuie sur les formules fondamentales des triangles déduites, d'après Lobatschewsky, de la surface hypothétique nommée horisphère. Or, pour être à l'abri de toute objection, il serait essentiel de prouver d'abord ces formules en n'utilisant que les figures élémentaires immédiatement réalisables, et c'est ce qu'ont essaye de faire M. Battaglini, Sulla Geometria immaginaria di Lobatschewsky (Giornale, 1867, p. 217), puis surtout, d'une façon très complète, M. Gérard pour la Géométrie lobatschewskienne (Thèse, p. 26 et suivantes), et M. Mansion pour la Géométrie riemannienne, dans un article 
inspiré d'après la méthode de M. Gérard et inséré aux Comptes rendus du troisième Congrès scientifique internationaldes catholiques (section des Sciences mathé matiques et naturelles, p. 1 2-25) ( ${ }^{1}$ ). Pour plus amples détails nous renverrons le lecteur à ces deux Mémoires, et nous nous bornerons à exposer ce qu'il y a de plus essentiel pour comprendre les résultats qui y sont déduits.

Soient $e$ la base des logarithmes népériens, $i$ le symbole imaginaire et $x$ un nombre ou argument quelconque positif ou négatif. Les fonctions dites circulaires

(1) $\cos x=\frac{e^{i x}+e^{-i x}}{2}, \sin x=\frac{e^{i x}-e^{-i x}}{2 i}, \operatorname{tang} x=\frac{1}{i} \frac{e^{i x}-e^{-i x}}{e^{i x}+e^{-i x}}$ telles que

$$
\cos ^{2} x+\sin ^{2} x=\mathrm{I}, \quad \operatorname{tang} x=\frac{\sin x}{\cos x},
$$

et les fonctions dites hyperboliques

(2) $\operatorname{ch} x=\frac{e^{x}+e^{-x}}{2}, \quad \operatorname{sh} x=\frac{e^{x}-e^{-x}}{2}, \quad$ th $x=\frac{e^{x}-e^{-x}}{e^{x}+e^{-x}}$ pour lesquelles

$$
\operatorname{ch}^{2} x-\operatorname{sh}^{2} x=\mathrm{I}, \quad \operatorname{th} x=\frac{\operatorname{sh} x}{\operatorname{ch} x}
$$

sont les seules dont nous ayons à faire emploi; l'équation $\cos x=0$ a une racine et une seule comprise entre I et 2 ; c'est $x=\frac{\pi}{2}$, telle que $\sin \frac{\pi}{2}=\mathrm{I}$, et $\sin x=\cos \left(\frac{\pi}{2}-x\right)$.

Prenons pour unité de comparaison une longueur arbitraire I. du plan; les trois côtés d'un triangle rectangle $A B C$ ont respectivement pour mesures

$$
a=\frac{\mathrm{BC}}{\mathrm{L}}, \quad b=\frac{\mathrm{AC}}{\mathrm{L}}, \quad c=\frac{\mathrm{AB}}{\mathrm{L}} ;
$$

chercher la relation qui existe entre les nombres $a, b, c$ est l'objet de la première question à résoudre. On y parvient $\mathrm{en}$ démontrant successivement les propositions que voici :

( $\left.{ }^{1}\right)$ Voir également Mathesis, février $188^{5}$, supplément.

IRIS - LILLIAD - Université Lille 1 
$I^{\circ}$ Si dans le triangle $\mathbf{M A} \mathbf{M}^{\prime}$, rectangle en $\mathbf{M}^{\prime}$, l'angle $\mathrm{A}$ est aigu et fixe, les rapports $\frac{\mathbf{A} \mathbf{M}^{\prime}}{\mathbf{A M}}$ et $\frac{\mathbf{M M}^{\prime}}{\mathbf{A} \mathbf{I}}$ ont des limites pour $\mathbf{A M}$ égal à zéro; on les appelle cosinus et sinus de l'angle $\Lambda$.

$2^{\circ}$ Si dans le quadrilatère ABCD les angles $A, B$ et $C$ sont droits, et $A C$ une longueur fixe, le rapport $\frac{C D}{A B}$ a une limite déterminée $\varphi(A C)$ pour $A B=$ o et $C D=0$.

$3^{\circ} \varphi(x)$ est une fonction continue satisfaisant à la définition

$$
\varphi(x-y)+\varphi(x+y)=2 \varphi(x) \cdot \varphi(y) .
$$

$4^{\circ}$ Entre les côtés d'un triangle rectangle dont $a$ est l'hypoténuse on a la relation

$$
\varphi(a)=\varphi(b) \cdot \varphi(c) \text {. }
$$

Soient $A_{1} B_{1} C_{1}$ et $A_{2} B_{2} C_{2}$ deux positions nouvelles successives $d u$ triangle $A B C$ oblenues en le faisant glisser d'abord de $A_{A_{1}=C C_{1}}$ le long de $A C_{1}$, puis de $B_{1} B_{2}=C_{1} C_{2}$ le long de $\mathrm{B}_{1} \mathrm{C}_{1}(f i g .8)$. On trace $\mathrm{BD}$ perpendiculaire à $\mathrm{B}_{1} \mathrm{C}_{1}, \mathrm{~B}_{1} \mathrm{D}_{1}$ perpendiculaire à $\mathrm{AB}, \mathrm{EE}_{1}$ perpendiculaire commune à $\mathrm{BC}$ et $\mathrm{B}_{1} \mathrm{C}_{1}$, et $\mathrm{HH}_{2}$ perpendiculaire commune à $\Lambda \mathrm{C}$ et $\Lambda_{2} \mathrm{C}_{2}$.

La figure montre aisément que les rapports $\frac{B D}{B B_{2}}$ et $\frac{B_{1} D_{1}}{B_{1} B_{2}}$ ont mêmes limites, ainsi que les rapports $\frac{\mathrm{EE}_{1}}{\mathrm{CC}_{1}}$ et $\frac{\mathrm{HL}_{\mathbf{Z}}}{\mathrm{C}_{1} \mathrm{C}_{2}}$.

\section{Fig. 8.}

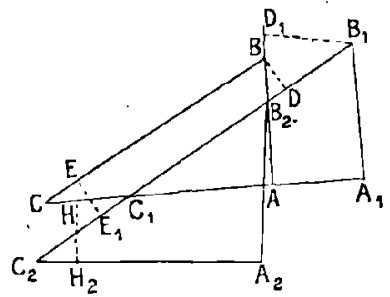

Donc,

$$
\lim \frac{B D}{\mathrm{EL}_{1}}=\lim \frac{\mathrm{B}_{1} \mathrm{D}_{1}}{\mathrm{AA_{1 }}} \times \lim \frac{B B_{2}}{\mathrm{HH}_{2}}
$$


$\mathrm{Or}$

$$
\begin{aligned}
\lim \frac{B D}{E E_{1}} & =\varphi(B C)=\varphi(a), \\
\lim \frac{B_{1} D_{1}}{A A_{1}} & =\varphi(A B)=\varphi(c), \\
\lim \frac{B B_{2}}{H I_{2}} & =\varphi(A C)=\varphi(b),
\end{aligned}
$$

ce qui démontre la proposition.

Les fonctions $\cos x$ et ch $x$ définies par les égalités (1) et (2) sont des cas particuliers évidents de la forme $\varphi$; réciproquement, la fonction o la plus générale ne peut avoir que l'une des deux formes

$$
\varphi(x) \equiv \cos (\lambda x), \quad \varphi(x) \equiv \operatorname{ch}(\lambda x),
$$

$\lambda$ désignant un multiplicateur convenable, mais qui garde une valeur déterminée et constante pour toute l'étendue du plan. Enfin, par sa définition même, la fonction $\varphi$ du plan riemannien ne peut dépasser i en valeur absolue, tandis que sa similaire sur le plan lobatschewskien est supérieure ou au moins égale à $\mathbf{I}$; pour ces motifs, les trois côtés d'un triangle rectangle riemannien sont lies par l'équation fondamentale

$$
\cos (\lambda a)=\cos (\lambda b) \cdot \cos (\lambda c),
$$

tandis que ceux d'un triangle rectangle lobatschewskien satisfont à l'équation de même forme

$$
\operatorname{ch}(\lambda a)=\operatorname{ch}(\lambda b) \cdot \operatorname{ch}(\lambda c) \text {. }
$$

Soit maintenant un triangle $\mathrm{AlBC}$ quelconque, dans lequel il y a lieu de supposer tout d'abord l'angle A aigu. Désignons encore par $a, b, c$ les mesures des trois côtés par rapport à l'unite arbitraire $\mathrm{L}$, et abaissons $\mathrm{BB}^{\prime}$ et $\mathrm{CC}^{\prime}$ perpendiculaires sur $A C$ et $A B$. La relation (5) appliquée aux quatre triangles rectangles de la figure donne les égalités

$$
\begin{aligned}
& \cos (\lambda a)=\cos (\lambda b) \cos (\lambda c)+\sin (\lambda b) \sin \left(\lambda \frac{A B^{\prime}}{\mathrm{L}}\right) \cos \left(\lambda \frac{\mathrm{BB}^{\prime}}{\mathrm{L}}\right), \\
& \cos (\lambda a)=\cos (\lambda b) \cos (\lambda c)+\sin (\lambda c) \sin \left(\lambda \frac{A \mathrm{C}^{\prime}}{\mathrm{L}}\right) \cos \left(\lambda \frac{\mathrm{CC^{ \prime }}}{\mathrm{L}}\right),
\end{aligned}
$$

IRIS - LILLIAD - Université Lille 1 
d'où, par comparaison,

$$
\frac{\operatorname{tang}\left(\lambda \frac{A B^{\prime}}{I}\right)}{\operatorname{tang}\left(\lambda \frac{A B}{L}\right)}=\frac{\operatorname{tang}\left(\lambda \frac{A C^{\prime}}{L}\right)}{\operatorname{tang}\left(\lambda \frac{A C}{L}\right)} .
$$

Or, $\mathrm{AB}$ et $\mathrm{AC}$ sont indépendantes, et la valeur commune de ces derniers rapports reste la même quand, $A B$ et l'angle $A$ demeurant fixes, on fait tendre $A G$ et $\mathrm{AC}^{\prime}$ vers zéro; dans ces conditions, le second rapport a une limite déterminée, égale, d'après les propriétés de la fonction circulaire, à celle du rapport $\frac{\Lambda \mathrm{C}^{\prime}}{\mathrm{AC}}$; cette limite est, par définition, le cosinus de l'angle $A$.

$5^{\circ}$ Si l'angle $A$ vaut $\frac{m}{n}$ d'angle droit, son cosinus et son sinus sont des fonctions circulaires du rapport $\frac{m}{n} \frac{\pi}{2}=A$; on peut ainsi les désigner par les notations $\cos A$ et $\sin A$.

Nous avons donc la relation

$$
\cos (\lambda a)=\cos (\lambda b) \cos (\lambda c)+\sin (\lambda b) \sin (\lambda c) \cos A \text {. }
$$

Quand A est un angle obtus, son cosinus est celui de son supplément pris avec le signe négatif; il a même sinus que ce supplément.

Pour un triangle quelconque lobatschewskien, on aurait de mèrne

$$
\operatorname{ch}(\lambda a)=\operatorname{ch}(\lambda b) \operatorname{ch}(\lambda c)-\operatorname{sh}(\lambda b) \operatorname{sh}(\lambda c) \cos A .
$$

Il est utile de remarquer dès à présent que la formule (7) est identique à celle de la Trigonométrie sphérique, indépendante du Postulatum d'Euclide, d'après une remarque at tribuée à Lagrange ( ${ }^{1}$ ). Taurinus avait pressenti la formule (8) en 1825 (Theorie der Parallellinien, 1825 ; Geometrice prima elementa, 1826 ).

Jusqu'ici, L et par conséquent $\lambda$ ont été laissés arbitraires ;

(') Conversation avec Brot rapportéc dans l'Essai critique sur les principes fondamentaux de la Gémétrie élementaire, par HoüsL.

IRIS - LILLIAD - Université Lille 1 
pour avoir sur le plan riemannien $\lambda=\mathrm{I}$, il faut remplacer $\mathrm{L}$ par la longueur $\mathrm{U}=\frac{2 \Delta}{\pi}$ qui est l'unité naturelle, ou paramètre du plan. $\Delta$ peut se construire, mais non $\mathrm{U}$.

Sur le plan lobatschewskien, nous admettrons avec Bolyai l'existence d'une longueur particulière $U$ qu'il appelle également unité naturelle des longueurs et qui correspond de son côté à l'hypothèse $\lambda=1$. $U$ peut se concevoir : $1^{\circ}$ comme limite de longueurs que l'on sail construire, $x^{\circ}$ comme égale à un arc d'horicycle dont on sait trouver les extrémités; mais il est impossible de tracer cette longueur mème.

Les éléments, angles et cótés d'un triangle rectangle sont liés par dix formules faciles à déduire de ( 7 ) et (8), et dont trois seulement, étant distinctes, suffisent à retrouver les autres. Voici un moyen mnémotechnique très commode pour les écrire ( $\left.{ }^{1}\right)$ : que l'on trace un pentagone ( $f i g .9$ ) et que

Fig. 9 .

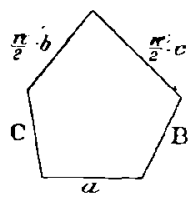

l'on inscrive sur ses côtés successifs, dans l'ordre indiqué,

$$
a, \quad \mathrm{~B}, \frac{\pi}{2}-c, \frac{\pi}{2}-b, \quad \mathrm{C} ;
$$

le cosinus de tout élément égale le produit des sinus des deux éléments non adjacents, ou le produit des cotangentes des deux éléments adjacents.

Dans cette règle, les cosinus, sinus et cotangentes des angles sont circulaires; ceux des longueurs sont circulaires on hyperboliques selon que le triangle est riemannien ou lobatschewskien. Les dix relations qu'elle donne font retrouver ensuite toutes celles d'un triangle quelconque.

(') Dù à Nepen ; consulter Dostor, Nouvelles Annales, r866, p. 4ı\{20, et Enseignement mathematique, rgor, p. 223-22í.

IRIS - LILLIAD - Université Lille 1 
24. Formules des quadrilatères. Constructions fondamentales. - Passons à un quadrilatère trirectangle ABCD ( $f i g$. 10),

Fig. Io.

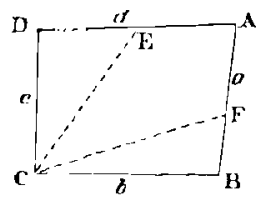

dans lequel l'angle $A$ est obtus ou aigu. Les côtés sont $\mathrm{AB}=a, \mathrm{BC}=b, \mathrm{CD}=c, \mathrm{AD}=d$. Ces éléments sont aussi exprimés en fonction les uns des autres par dix relations, parmi lesquelles trois seulement sont distinctes. Une règle mnémotechnique semblable à celle du paragraphe précédent les contient aussi; il faut cette fois écrire sur les côtés du pentagone, dans cet ordre, $\mathrm{A}, \frac{\pi}{2}-a . b, c, \frac{\pi}{2}-d$. Il est bon de noter toutefois une exception; sur le plan riemannien, la règle appliquée à la lettre donnerait

$$
\cos \mathbf{A}=\sin b \sin c, \quad \cos \mathbf{A}=\operatorname{tang} a \operatorname{tang} d,
$$

tandis que les formules véritables sont

$$
\cos \mathrm{A}=-\sin b \sin c, \quad \cos \mathrm{A}=-\operatorname{tang} a \operatorname{tang} d .
$$

Tout triangle rectangle et tout quadrilatère tricectangle peuvent se construire avec la règle et le compas quand on connaît en grandeur deux quelconques de leurs éléments. Bolyai et, d'après lui, M. Gérard ont donné la solution de quelques-uns de ces problèmes; nous avons repris et complété leur étude dans notre Mémoire de Géométrie analytique non euclidienne déjà cité (\$ 2,p. 5-I 4$)$. En voici les principaux points pour le plan Jobatschewskien.

$S i d u$ point $C$ comme centre ( $f g$. 10 ), avec $A B>C D$ et $\mathrm{AD}>\mathrm{CB}$ comme rayons, nous decrivons deux arcs de circonférence coupant respectivement $A D$ en $E$ el $A B$ eu $F$, les angles CFB, CED, BCE, DCF sont les angles de parallelisme répondant aux distances respectives $a, b, c, d$; les longueurs BF et DE sont égales et leur angle de parallélisme 
égale A. De lá vient que CF est parallèle à DA et que CE est aussi parallèle à $B A$. De ces remarques nous déduisons le moyen simple :

- De mener par un point C la parallèle CE à une droite $\mathrm{BA}$, ou, ce qui est équivalent, de construire l'angle de parallélisme $\mathrm{BCE}$ répondant à une distance donnée Cl3; on sait que Lobatschewsky désigne cet angle par la notation II (CB) $\left({ }^{1}\right)$ et le détermine par l'équation $\left({ }^{2}\right)$

$$
\operatorname{tang} \frac{\mathrm{I}}{2} \Pi(\mathrm{CB})=e^{-\frac{C \mathrm{~B}}{\mathrm{U}}}
$$

d'où l'on déduit aisément

$$
\cos I I(C B)=\operatorname{th} \frac{C B}{U} ;
$$

$2^{\circ}$ De construire inversement Ia longueur CB qui répond à un angle de parallélisme donné $B C E$, ce qui revient à tracer la droite $A B$ perpendiculaire à $C B$ et parallèle à $C E$;

$3^{\circ}$ De tracer la perpendiculaire commune CB aux droites $\mathrm{CD}$ et $\mathrm{B} \Lambda$, et le triangle rectangle $\mathrm{BFC}$ dont les deux angles aigus $B F C$ et $B C F$ sont donnés.

Avec ce qui précède, nous avons le moyen de construire les points de rencontre d'un horicycle ou d'un hypercycle avec une droite, soit encore les points communs à ces courbes; puis nous pouvons construire toutes les longueurs qui ont pour sinus hyperboliques des nombres commensurables et, en particulier, celles dont le sinus est entier; l'unité naturelle de Bolyai est inaccessible, mais comme c'est une limite de longueurs à sinus commensurables, nous pouvons en approcher d'autant que nous voudrons et la représenter également par un arc d'horicycle tel que la tangente à une extrémité est parallèle au rayon de l'autre. Notons ici en passant cette singulière propriété de l'horicycle, qui semble être la contre-partie de celle du cercle trigonométrique de rayon égal à I dans la Géométrie euclidienne : la longueur d'un arc OM est à la fois le sinus hyperbolique de la moitié $m \mathrm{M}$ de la corde $\mathrm{MM}^{\prime} q u i$ sous-

(') Recherches géométriques sur la theorie des paralleles, $\mathrm{n}^{\circ} 16$. ( $\left.{ }^{2}\right)$ Ibid., n० 36 .

IRIS - LILLIAD - Université Lille 1 
tend l'arc double $\mathrm{MOM}^{\prime}$, et la tangente hyperbolique de la portion $\mathrm{OT}$ de tangente géométrique en $\mathrm{O}$ comprise entre ce point et l'extrémité $\mathrm{T}$ du rayon passant par $\mathrm{M}$.

Nous pouvons reproduire des constructions de même nature pour le quadrilatère trirectangle riemannien et, par conséquent, pour le quadrilatère sphérique; seulement, comme dans la figure i I nous avons $c>a$ et $b>d$, il nous

Fig. I $\mathbf{r}$.

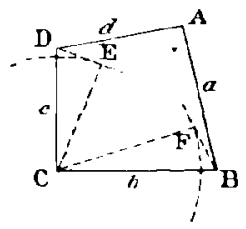

faut de C comme centre, avec des rayons égaux à $a$ et $d$, décrire des circonférences et leur mener des points $B$ et $D$ les tangentes BF et DE. Si aux deux extrémités d'une longueur $l$ on ćlève les perpendiculaires, elles forment en se coupant un angle déterminé qui correspond à $l$; les angles $A B F, A D E, B C F$, DCE correspondent de cette façon aux longueurs respectives $a, b, c, d$; et de plus, aux longueurs égales BF, DE correspond l'angle A- $\mathrm{I}^{\mathrm{dr}}$. Nous saurons ainsi, par des tracés faciles, trouver la longueur à laquelle correspond un angle donné, ou vice versa, puis construire telle fraction qu'on veut de $\Delta$, déterminer la perpendiculaire commune à deux droites, etc. $\left({ }^{1}\right)$.

Ainsi la Géométrie non euclidienne se suffit à elle-même; avec le secours de la règle, de l'équerre et du compas, elle peut construire toutes les figures planes sur lesquelles elle raisonne et s'élever ensuite par leur moyen à la conception des figures correspondantes de l'espace; de la sorte, aucun doute ne subsiste plus dans l'esprit sur l'existence réelle de ces figures, mais qu'on nous comprenne bien. Nous ne songeons pas le moins du monde, comme le dit plaisamment

( $\left.{ }^{1}\right)$ Consulter Constructions spheriques à la règle et au compas (Mathesis, 1899 , p. 81-85).

IRIS - LILLIAD - Université Lille 1 
LA TRIGONOMÉTRIE.

M. Andrade ( ${ }^{1}$ ), à interdire à nos équerres le dessin des carrés; si nous traçons un quadrilatère qui ait trois angles droits, l'expérience seule peut nous éclairer sur la valeur du quatrième, et, tant que nous ne sommes pas fixés à cet égard, celte valcur doit être regardée par nous comme purement conventionnelle; mais il n'importe, les tracés subséquents pourront toujours s'achever d'une façon ou d'une autre.

(1) Euclidien et non euclidien (Ens, math., r9oo). 


\section{CHAPITRE VI.}

MESURE DES AIRES ET VOLUMES.

20. Aires planes, triangle et polygone. - Dans un triangle non euclidien, la difference entre deux droits et la somme des angles s'appelle excès ou déficit suivant le sens dans lequel elle se manifeste. C'est à Lambert que l'on doit de connaître Ja relation simple qui existe entre l'aire du triangle et cet ètement géométrique, et l'on peutétablir leur proportionnalité par une méthode élémentaire qui n'emploie que des décompositions de figures équivalentes en parties respectivement superposables.

I. Deux quadrilatères trirectangles qui ont le quatrième angle et un côté adjacent respectivement égaux chacun à chacun sont superposables. Cette proposition, qui resulte de ce que les seconds côtés du quatrième angle sont aussi égaux d'après la règle mnémotechnique du pentagone, peut s'établir directement comme il suit.

Soient ABCal, $A^{\prime} B^{\prime} C^{\prime} D^{\prime}$ les deux quadrilatères trirectangles dans lesquels les angles aigus ou obtus $\mathbf{A}$ et $\mathbf{A}^{\prime}$ sont égaux, ainsi que les côtés adjacents $\mathrm{AB}$ et $\mathrm{A}^{\prime} \mathrm{B}^{\prime}$ ( $f_{g}$. 12). Si

Fig. 12 .
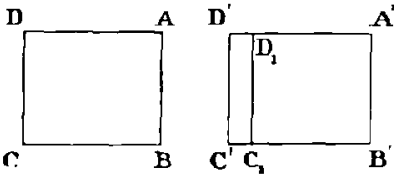

nous transportons le premier sur le deuxième de façon à faire coincider les éléments respectivement égaux, il vient prendre

Scientia, $n^{\circ} 15$.

IRIS - LILLIAD - Université Lille 1 
la position $\mathrm{A}^{\prime} \mathrm{B}^{\prime} \mathrm{C}_{1} \mathrm{D}_{1}$; or, si $\mathrm{C}_{1} \mathrm{D}_{1}$ était distincte de $\mathrm{C}^{\prime} \mathrm{D}^{\prime}$, le quadrilatère $\mathrm{C}_{1} \mathrm{D}_{1} \mathrm{D}^{\prime} \mathrm{C}^{\prime}$ aurait ses quatre angles droits, ce qui est contre l'hypothèse. Donc $\mathrm{C}_{1} \mathrm{D}_{1}$ coïncide avec $\mathrm{C}^{\prime} \mathrm{I}^{\prime}$.

II. Tout triangle ABC est équivalent au double d'un quadrilatère trirectangle, et la somme des angles $\mathrm{A}, \mathrm{B}, \mathrm{C}$ est égale au double de l'angle non droit du quadrilatère.

En nous reportant à la figure 6 , nous voyons effectivement Fig. 6.

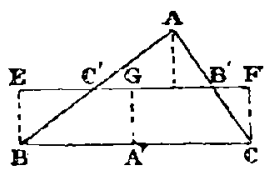

que le triangle $A B C$ est équivalent au quadrilatère birectangle et isoscèle $\mathrm{BLCF}$, à cause des égalités de triangles $\Lambda \mathrm{DC}^{\prime}$ et $\mathrm{BEC}^{\prime}, \mathrm{DDB}^{\prime}$ et $\mathrm{CFB}^{\prime}$. D'ailleurs, ce quadrilatère vaut à son tour le double du quadrilatère trirectangle BEGA', dans lequel l'angle $A^{\prime} B E$ est aigu ou obtus suivant l'espèce de Géométrie. Soit a $\Sigma$ la somme des angles $A, B$, C; nous avons

$$
2 \mathrm{~L}=\mathrm{BAD}+\mathrm{DAC}+\mathrm{ABC}+\mathrm{ACB}=\mathrm{EBC}+\mathrm{BCF}=2 \mathrm{~A}^{\prime} \mathrm{BE} .
$$

Nous remarquerons que la ligne $\mathrm{B}^{\prime} \mathrm{C}^{\prime}$ est égale à la somme des lignes B'F el EC', c'est-ádire à la moitié de EF ou à EG.

III. Deux triangles ABC, IBC qui ont même base BC et des sommes angulaires égales sont équivalents.

Fig. 13.

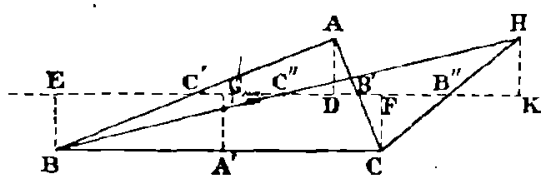

Soit 2 $\mathbf{\Sigma}$ la somme angulaire commune; d'après II, les deux triangles sont respectivement équivalents aux doubles des

IRIS - LILLIAD - Université Lille 1 
quadrilatères trirectangles $\mathrm{A}^{\prime} \mathrm{BEG}, \mathrm{A}^{\prime} \mathrm{BE} \mathrm{E}_{1} \mathrm{G}_{1}$ ( fig. I 3 ) qui ont le côté $\Lambda^{\prime} \mathrm{B}$ commun et les angles non droits $\mathrm{A}^{\prime} \mathrm{BE}$, $\mathrm{A}^{\prime} \mathrm{BE}_{1}$ égaux à $\Sigma$; or, en vertu de $\mathrm{I}$, ces quadrilatères doivent coïncider, donc ABC et. HBC sont équivalents.

Réciproquement, deux triangles équivalents $\mathrm{ABC}$, HBC de même base BC ont aussi même somme angulaire $2 \mathrm{\Sigma}$.

Remarque a. - Dans tous les triangles équivalents de méme base $\mathrm{BC}$, la droite qui joint les milieux des deux cótés a une longueur constante, car la figure 13 donne

$$
\mathbf{B}^{\prime} \mathrm{C}^{\prime}=\mathrm{B}^{\prime \prime} \mathrm{C}^{\prime \prime}=\mathrm{GE} \text {. }
$$

Remarque b. - Le lieu géométrique des sommets des triangles équivalents et de méme base est un hypercycle. Tirons, en effet, dans la figure $13, \mathrm{HK}$ perpendiculaire sur EF, nous avons $H K=\mathrm{AD}=\mathrm{BE}$; donc le lieu géométrique des sommets A, I, etc. est la branche d'hypercycle qui a pour axe EF, pour équidistance $B E$, et qui ne passe pas par les points $B$ et $C$.

Cette proposition a été démontrée par Lexell pour les triangles sphèriques.

Remarque c. - On peut toujours remplacer un triangle donné $\mathrm{ABC}$ par ùn triangle équivalent $\mathrm{HBC}$ ayant même base BC, et dans lequel l'un des angles adjacents, BCII par exemple, est égal à un angle donné. En effet, construisons l'angle $\mathrm{BCH}$ égal à l'angle donné, faisons couper $\mathrm{B}^{\prime} \mathrm{C}^{\prime}$ avec $\mathrm{CH}$ en $\mathrm{B}^{\prime \prime}$ et prenons $\mathrm{CH}={ }_{2} \mathrm{CB}^{\prime \prime}$; l'opération ne peut évidemment se faire que si l'angle donné est inférieur à $\Sigma$ augmenté de l'angle de parallélisme qui correspond à CF.

Remarque d. - On peut toujours remplacer un triangle donné $\mathrm{ABC}$ par un triangle équivalent $\mathrm{HBC}$ ayant même base BC, et dans lequel l'un des côtés adjacents, $\mathrm{BH}$ par exemple, ail une longueur donnée l supérieure à l'un des cótés BA. Il suffit pour cela de faire couper $\mathrm{B}^{\prime} \mathrm{C}^{\prime}$ avec la circonférence de centre $\mathrm{B}$ qui a pour rayon $\frac{l}{2}$; le point $\mathrm{C}^{\prime \prime}$ d'intersection existe toujours puisque $\mathrm{BC}^{\prime \prime}$ est plus grande que $\mathrm{BC}^{\prime}$, et il ne reste plus qu'à prendre $\mathrm{BH}=2 \mathrm{BC}^{\prime \prime}$.

IRIS - LILLIAD - Université Lille 1 
IV. Deux triangles $\mathrm{ABC}, \mathrm{A}^{\prime} \mathrm{B}^{\prime} \mathrm{C}^{\prime}$ de même somme angulaire sont équivalents.

Soit $l$ une longueur plus grande que les côtés de ces deux triangles. D'après la remarque $d$, chacun d'eus peut être remplacé par un triangle équivalent et de mème somme angulaire ayant un còté égal à $l$; mais les deux nouveaux triangles sont équivalents d'après la proposition directe III ; donc $\mathrm{ABC}$ et $\mathrm{A}^{\prime} \mathrm{B}^{\prime} \mathrm{C}^{\prime}$ sont équivalents.

Réciproquement, deux triangles $\mathrm{ABC}, \mathrm{A}^{\prime} \mathrm{B}^{\prime} \mathrm{C}^{\prime}$ équivalents ont même somme angulaire.

Conoldane. - Pour que deux triangles soient équivalents, il faut et il suffit qu'ils aient même excès ou mếme déficit angulaire.

Pour faire la somme de deux triangles, il suffit de les transformer d'abord en deux triangles rectangles qui leur soient respectivement équivalents et qui aient un côté de l'angle droit égal à une longueur donnée $l$, puis d'accoler ces deux derniers suivant le cóté commun; le triangle total a une somme angulaire égale à la réunion de leurs sommes angulaires partielles diminuée de deux droits; donc :

V. Si trois angles $\mathrm{ABC}, \mathrm{A}^{\prime} \mathrm{B}^{\prime} \mathrm{C}^{\prime}, \mathrm{A}^{\prime \prime} \mathrm{B}^{\prime \prime} \mathrm{C}^{\prime \prime}$ sont tels que le troisième est équivalent à la somme des deux premiers, son excès ou déficit angulaire est aussi égal à la somme de leurs excès ou déficits.

Le résumé de toutes les propositions précédentes se trouve contenn dans le théorème général que voici :

VI. Deux triangles quelconques sont proportionnels à leurs excès ou déficits angulaires.

Ou, en choisissant convenablement les unités :

La mesure de l'aire d'un triangle est égale à celle de son excès au déficit angulaire.

Ce théorème s'applique à un polygone convexe quelconque; pour le vérifier, on peut remarquer tout d'abord que, si le polygone est effectivement décomposé en triangles par des

IRIS - LILLIAD - Université Lille 1 
lignes joignant ses $n$ sommets à un mème point intérieur, la réunion des sommes angulaires de tous ces triangles est égale à la somme angulaire du polygone augmentée de quatre droits. Appelons excès ou déficit angulaire du polygone la différence entre $2 n-1$ droits et la somme de ses angles; cet excès ou déficit est donc égal à la somme des excès ou déficits des $n$ triangles de décomposition, ce qu'il fallait prouver. On sait d'ailleurs construire un triangle équivalent à un polygone dooné et ayant aussi même excès ou déficit que le polygone. En effet, soit ABCI)E le polygone donné de $n$ côtés $\left(f i_{g}\right.$. 14). Soient $M$ et $\mathrm{N}$ les milieux des côtés con-

Fig. 14.

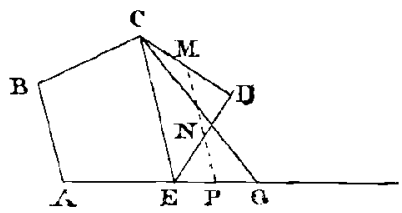

sécutifs $C D, D E$, la ligne $\mathrm{MN}$ rencontre le côté $\mathrm{AE}$ prolongé au point $P$; prenons $E G={ }_{2} E P$ et tirons $C G$. Les triangles DCE et GCE sont équivalents, donc le polygone proposé est aussi équivalent au polygone ABCG qui a $n$ - 1 côtés; mais la somme angulaire de ce dernier est égale á celle du premier moins deux droits, donc leurs excès ou déficits sont égaux.

D'un polygone de $n-\mathrm{r}$ côtés, on passe ensuite à un polygone de $n-2$ côtés, et ainsi de suite jusqu'au triangle. Il est intéressant de remarquer que la construction de la figure I4 est aussi textuellement celle de la Géométrie euclidienne.

Fixons maintenant les unités. Souvent on compare les triangles à celui daus lequel la somme angulairc est un nombre entier de droits. Sur le plan riemannien, c'est le triangle trirectangle dont l'excès vaut ur droit; sur le plan lobatschewskien, c'est le triangle équilatéral dont les angles sont égaux à un tiers de droit, el le déficit de ce dernier égale aussi un droit. En ce cas, la mesure d'un triangle égale le rapport de son excès ou déficit à l'angle droit, et l'aire du plan riemannien entier est mesurée par 8 . Mais ces unités théoriquement. simples ne sont pas commodes pour l'évaluation des aires 
terminées par des courbes, aussi préfère-t-on leur en substituer d'autres plus rationnelles.

La longueur d'une circonférence de rayon $R$, estimée par rapport à l'unité naturelle $U$, est $2 \pi \sin R$ ou $2 \pi \operatorname{sh} R$ (Lobatschewsky). Si l'on remplace $R$ par $\Delta$ ou par la longueur $V$ qui répond à l'angle de parallélisme $\frac{1}{2}$ droit ( $\$ 24$, construction $2^{\circ}$ ), on a deux circonférences particulières, dont la première n'est autre que la droite riemannienne, et dont les longueurs sont mesurées par $2 \pi$. L'angle au centre qui sur chacune d'elles intercepte un arc de longueur ćgale à $U$ vaut $\frac{1}{\pi} 2$ droits; il convient de le choisir pour nouvelle unité angulaire. Je triangle isoscèle formé par cet angle, avec ses deux côtés adjacents égaux à $\Delta$, et le côté opposé égal à $U$, est la nouvelle unité d'aire riemannienne. Pour définir l'unité nouvelle du plan lobatschewskien, concevons une droite égale à $\mathrm{U}$, les perpendiculaires à ses extrémités, et l'hypercycle de hauteur V. La portion de plan comprise entre ces lignes peut être regardée comme la limite de $2 n$ quadrilatères trirectangles égaux dans lesquels, l'angrle aigu étant désigné par $\boldsymbol{x}$, un des côtés de cet angle égale $V$, et le côté perpendiculaire au précédent égale $\frac{\mathrm{U}}{2 n}, n$ croissant indéfiniment. On a donc

$$
\operatorname{tang} \alpha=\operatorname{coth} \frac{\mathrm{L}}{2 \pi} .
$$

Par conséquent, l'aire totale des a $n$ quadrilatères, mesurée avec l'ancienne unité, a pour expression $S=2 n$ (I droit $-\alpha$ ), ce qui peut s'écrire :

$$
\mathrm{S}=2 n\left[\operatorname{arctang}\left(\operatorname{th} \frac{\mathrm{U}}{2 n}\right)\right] .
$$

Lorsque $n$ croît indéfiniment, $S$ a pour limite $\frac{2}{\pi}$ droits. C'est l'aire de la figure mixtiligne choisie pour nouvclle unité.

Moyennant ces changements, l'aire d'un cercle de rayon $B$ devient

$$
A=4 \pi \sin ^{2} \frac{R}{2} \quad \text { ou } \quad A=4 \pi \operatorname{sh}^{2} \frac{R}{2} ;
$$

IRIS - LILLIAD - Université Lille 1 
celle du trapèze hypercyclique de base $b$ et d'équidistance $p$ est

$$
\mathbf{A}=\boldsymbol{b} \sin p \quad \text { ou } \quad \mathbf{A}=\boldsymbol{b} \operatorname{shp},
$$

et le plan riemannien entier a pour surface $4 \pi$.

26. Aires des surfaces courbes. - Lobatschewsky ( $M e^{\prime}-$ moires de l'Université de Kazan), et quelques-uns de ses continuateurs parmi lesquels il convient de citer surtout M. Story, On the non Euclidean geometry (Journal de Silvester, 1882 ), et M. Simon, Détermination des aires et volumes en Géométrie non euclidienne (Math. Annalen, I $89^{3}$ ), ont publié des recherches originales sur la mesure des aires et des volumes des corps ronds. Voici, énoncés de la manière la plus simple, et complétés en quelques points, les principaux théorèmes auxquels leurs résultats on $t$ conduit.

L'aire latérale du tronc de cône (ou du cône) égale le produit de la circonférence moyenne par le double sinus de la demi-arêtc.

L'aire de la zone sphérique comprise entre l'équateur et un petit cercle concentrique égale le produit de la circonférence d'un grand cercle par le sinus distance de l'extrémité de l'arc mobile au plan de l'équateur.

L'aire de la sphère égale celle du cercle de rayon double. L'aire latérale engendrée par un arc d'hypercycle d'équidistance $r$ tournant autour de son axe égale le produit de la circonférence de rayon $r$ par la longueur de l'arc générateur.

Si en tous les points d'une aire plane limitée $A$ on élève des perpendiculaires égales à $p$, le lieu de leurs extrémités est une portion limitée $\mathrm{A}^{\prime}$ d'hypersphère (ou surface équidistante), égale au produit de A par le carré du cosinus de l'équidistance.

27. Volumes. - Considérons un élèment superficiel infiniment petit $d \mathrm{~A}$ de forme quelconque. Par chacun des points de son contour élevons sur le plan qui le renferme des perpendiculaires égales et de longueur infiniment petite $d h$; nous enfermons ainsi un solide élémentaire dont nous conviendrons de dire que le produit $d \mathrm{~A} d h$ mesure le volume avec une certaine unité. Tout corps solide de dimensions 
finies peut se regarder comme une somme d'éléments pareils en nombre indéfini et la formule

$$
\mathrm{V}=\iint d \mathbf{I} d h
$$

fait connaître l'expression générale conventionnelle des volumes en Géométrie non euclidienne. F́numérons les principaux théorèmes auxquels cette notion nous conduit.

Soit A une portion d'aire de la sphère de rayon $r$; le volume du secteur sphérique correspondant est

$$
\mathrm{V}=\frac{\mathrm{A}}{2 \sin ^{2} r}\left(r-\frac{1}{2} \sin 2 r\right) \quad \text { ou } \quad \mathrm{V}=\frac{\mathrm{A}}{2 \operatorname{sh}^{2} r}\left(\frac{1}{2} \operatorname{sh} 2 r-r\right) .
$$

Le volume de la sphère est donc

$$
\mathrm{V}=2 \pi\left(r-\frac{\mathrm{I}}{2} \sin 2 r\right) \quad \text { ou } \quad \mathrm{V}=2 \pi\left(\frac{\mathrm{I}}{2} \operatorname{sh} 2 r-r\right) .
$$

Soient $h$ la hauteur, $d$ l'arète latérale, $\theta$ le demi-angle générateur d'un cône de révolution, le volume de ce cône a pour expression

$$
\mathrm{V}= \pm \pi(h-d \cos \theta),
$$

le signe + s'appliquant à la Géomètrie riemannienne, et le signe - à la lobatschewskienne (1). Cette formule est vraie également du tronc de cône, et conduit à un théorème singulier dont nous signalerons une intéressante application dans notre dernier Chapitre:

La projection d'un segment de droite sur un axe est supérieure, égale, ou inférieure au produit du segrnent par le cosinus de l'angle d'inclinaison, suivant que la figure est riemannienne, euclidienne, ou lobatschewskienne. En Géométrie générale, la différence du produit à la projection est proportionnelle au volume de révolution engendré par le segment; en Géométrie euclidienne, cette différence

(') Lobatschewsky, Crelle, t. 27. - Max Simon, Math. Annalen, y 893 . - Bниванг, Les cosegments et les volumes en Géométrie non euclidienne (Société des Sc. phys. et nat. de Bordeaux, 1902).

IRIS - LILLIAD - Université Lille 1 
est nulle, et par suite le volume conventionnel exprimé par I'intégrale double (9) vaut zéro. Pour comprendre la raison de ce fait, il faut remarquer d'abord que, si dans les équations (5) et (6) du $\$ 23$ nous faisons croître au delà de toute grandeur l'unité fondamentale linéaire U, en utilisant les développements en série des fonctions cos et ch, ces équations tendent vers l'équation limite du théorème de Pythagore:

$$
a^{2}=b^{2}+c^{2}
$$

donc la Géométrie euclidienne doit être considérće comme un cas limite commun aux deux Géométries non euclidiennes. Ceci posé, quand U croît indéfiniment, l'unite de volume, qui varie évidemment dans le même sens que $U$, croîl aussi au delà de toute grandeur, et dans ces conditions le volume du cône engendré par un segment quelconque fini n'est plus qu'un infiniment petit de l'unité de volume elle-même. Pour ćluder la difficulté, et savnir ce que devient l'intégrale (9) avec une autre unité demeurant finie, nous poserons, par exemple, en Géométrie riemannienne

$$
\operatorname{tang} h=\operatorname{tang} d \cos \theta
$$

par l'introduction des fonctions tang $h$ et $\operatorname{tang} d$ développées en séries, nous obtiendrons alors

$$
h-d \cos \theta=\frac{\frac{1}{3} h\left(d^{2}-h^{2}\right)+\frac{2}{1.5} h\left(d^{4}-h^{4}\right)+\frac{17}{315} h\left(d^{6}-h^{6}\right)+\ldots}{1+\frac{1}{3} d^{2}+\frac{2}{15} d^{4}+\frac{17}{315} d^{6}+\ldots} .
$$

Soit L une longueur arbitraire; si nous prenons pour unité d'aire le trapèze hypercyclique qui a $L$ pour base et pour équidistance, et pour unité de volume le solide qui a pour volume le produit de cette aire par L, la nouyelle expression du volume du cône est égale à celle de la formule (ro) multipliée par le rapport

$$
\frac{\mathrm{U}^{2}}{\mathrm{~L}^{2} \sin \left(\frac{\mathrm{I}}{\mathrm{U}}\right)}
$$

IRIS - LILLIAD - Université Lille 1 
c'est-à-dire

$$
V^{\prime}=\pi \frac{\frac{1}{3} \frac{H}{L}\left(\frac{\mathrm{D}^{2}}{\mathrm{~L}^{2}}-\frac{\mathrm{H}^{2}}{\mathrm{I}^{2}}\right)+\frac{2}{\mathrm{I} 5} \frac{\mathrm{H}}{\mathrm{L}}\left(\frac{\mathrm{D}^{4}}{\mathrm{U}^{2} \mathrm{~L}^{2}}-\frac{\mathrm{H}^{4}}{\mathrm{U}^{2} \mathrm{~L}^{2}}\right)+\ldots}{\mathrm{I}+\frac{\mathrm{I}}{3} \frac{\mathrm{D}^{2}}{\mathrm{U}^{2}}+\frac{2}{\mathrm{I} 5} \frac{\left(\frac{\mathrm{L}}{\mathrm{U}}\right)}{\mathrm{U}^{4}}+\ldots} \times \frac{\sin \left(\begin{array}{l}
\mathrm{L} \\
\mathrm{C}
\end{array}\right)}{,},
$$

et, quand L demeurant fixe $U$ devient infini, elle a pour limite finie

$$
\mathrm{V}^{n}=\frac{\mathbf{1}}{3} \pi \frac{\mathrm{H}}{\mathrm{L}}\left(\frac{\mathrm{D}^{2}}{\mathrm{~L}^{2}}-\frac{\mathrm{H}^{2}}{\mathrm{~L}^{2}}\right)=\frac{\mathrm{I}}{3} \pi \frac{\mathrm{H}}{\mathrm{L}} \frac{\mathrm{R}^{2}}{\mathrm{~L}^{2}},
$$

qui mesure précisément le cône euclidien de hauteur $\mathrm{H}$ et de rayon de base $R$.

le volume de l'hypercycloïde de révolution égale le produit de sa hauteur par le quart de l'aire du cercle de rayon double, ou encore le produit de l'aire latérale par la demitangente (circulaire ou hyperbolique) du rayon.

Pour évaluer un volume de révolution quelconque, considérons une ordonnée $m M$ de la courbe méridienne $G$; la tranche infiniment mince de volume qui a pour épaisseur $d X$ le long de l'axe appartient à un hypercycloüde ayant $m \mathrm{M}$ pour rayon; donc, $y$ désignant le sinus de l'ordonnée,

$$
\mathrm{V}=\pi \int_{y_{\mathrm{a}}}^{y_{1}} y^{2} d \mathrm{X}
$$

est l'expression du volume fini limitè par les parallèles $m_{0} \mathbf{M}_{0}$ et $m_{1} \mathbf{M}_{1}$.

L'espace riemannien entier peut être évalué à deux points de vue. En premier lieu, tout plan le partage en deux parties cgales dont chacune est une sphère de rayon $\Delta$ ou $\frac{\pi}{2}$ et a pour volume $\pi^{2}$. Considérons en outre un hypercycloïde de révolution de rayon $r$ ayant pour axe la droite $\mathrm{AB}$; tout point $\mathrm{M}$ de son hypercycle méridien est à la distance $\Delta-r$ de la ligne $\Lambda^{\prime} B^{\prime}$ réciproque de $A B$, et par suite la surface externe de ce premier solide doit être aussi regardée comme formant la surface interne d'un deuxième hypercycloïde complémentaire. L'espace ricmannien entier est la somme de leurs volumes, qui sont analogues à des tores s'emboîtant récipro-

IRIS - LILLIAD - Université Lille 1 
quement; il a donc pour expression

$$
V=2 \pi^{2} \sin ^{2} r+2 \pi^{2} \cos ^{2} r=2 \pi^{2} .
$$

La question du volume des polyèdres, et en particulier du tétraèdre, est beaucoup plus difficile à résoudre. Elle a occupé Lobatschewsky, Gauss et Jean Bolyai dont les solutions ont été récemment retrouvées dans un écrit posthume du géomètre hongrois ( $\left.{ }^{1}\right)$; M. Simon lui consacre une partie de son Mémoire sur les volumes $\left({ }^{2}\right)$. De même que l'aire du triangle est une fonction de ses angles, le volume du tétraèdre est une fonction de ses dièdres, ainsi qu'il est prouvè dans notre Travail sur Les cosegments et les volumes $\left({ }^{3}\right)$, où nous sommes conduits à ce résultat :

Le volume d'une pyramide est égal à plus ou moins la moitié de la somme obtenue en ajoutant:

I Les produits de chaque arête latérale par le dièdre correspondant;

$2^{\circ}$ Le produit de la hauteur par zéro ou par $2 \pi$ suivant qu'elle est extérieure ou intérieure ;

$3^{\circ}$ Certaines séries convergentes relatives aux faces latérales.

(') P. SтÄскец, Untersuchungen aus der absoluten Geometrie, aus Johann Bolyai's Nachlass . (Math. und Naturwiss. Berichte aus Ungarn, Igo2, p. 280-307).

(") Math. Annalen, i8g3.

(3) Mém. de la Soc. des Sc. phys. et nat. de Bardeaux, $19^{02 .}$ 


\section{CHAPITRE VII.}

LES CONTRADICTEURS DE IA GÉométhiE NON EUGLidieNNE.

28. Objections principales. - Par les études qui précèdent, on voit que, s'il est possible de pousser aussi loin que l'on veut, et sans aucun empêchement logique, l'analyse des figures géométriques non euclidiennes, quelques-unes d'entre elles présentent des propriétés assez étranges quand on les compare aux propriétés des figures analogues de la Géométrie usuelle. Aussi des contradicteurs ingénieux n'ont-ils manqué d'en prendre texte pour attaquer les doctrines de Lobatschewsky, Bolyai et Riemann, afin den conclure ensuite, par voie d'exclusion, la vérité du Postulatum d'Euclide, indémontrable par des moyens directs. Ne nous occupant que des arguments qui valent scricusement la peine d'ètre relevés, nous les résumerons ainsi qu'il suit :

$I^{0}$ Les droites riemanniennes ont toutes les propriétés des grands cercles d'une sphère euclidienne, et la trigonométrie des triangles riemanniens est identique au fond avec celle des triangles sphériques; donc le plan riemannien ne fait qu'un avec une surface sphérique euclidienne ( $\left.{ }^{1}\right)$.

En I 825, 'Taurinus conjecture hardiment qu'il peut exister certaines surfaces courbes le long desquelles il y a des lignes courbes particulières jouissant de propriétés analognues à

(1) F. DAtge, Cours de méthodologie mathèmatique; Lettre à M. P. Mansion (Mathesis, janvier $\mathrm{rgg} 6$ ); Note Sur l'interpretation d'un théorème de Géometrie riemannienne (Mathesis, janvier 1898 ).

Delbcar, L'ancienne et les nouvelles Géométries (Revue philosophique, avril $x 894$ ).

Lechalas, Identité des plans riemanniens et des sphères d'Euclide (Ann. de la Soc. scient. de Bruxelles, 1896 ); Introduction à la Géometrie générale, Gauthier-Villars, 1904 .

IRIS - LILLIAD - Université Lille 1 
celles des droites dans le plan, à part celle qui est renfermée dans le $5^{\mathrm{e}}$ postulat. Beltrami démontre plus tard dans son Essai d'interprétation de la Géométrie lobatschewskienne que celte conjecture était parfaitement fondèe. Si l'on fait tourner autour de son axe ou asymptote la tractrice, appelée aussi courbe des tangentes égales $\left({ }^{1}\right)$, la surface de révolution ainsi engendrée est une pseudo-sphère; elle a une courbure totale constante, mais négative; ses ligues géodésiques s'entre-croisent de façon à former des triangles où la somme angulaire est toujours inférieure à deux droits; donc le plan lobatschewskien est identique à une pseudo-sphère, et les droites lobatschewskiennes ne sont que des geodesiques de cette surface.

S'il en est véritablement ainsi, il est impossible de se représenter un espace riemannien ou lobatschewskien ; la Géométrie non euclidienne à deux dimensions s'explique tout naturellement, mais il ne saurait exister de Géométrie semblable à trois dimensions.

$2^{\circ}$ Les nombres n'ont par cux-mêmes aucune siguification concrìte, et il serait absurde d'affirmer, par exemple, que la connaissance d'un nombre $t$ suffit seule à déterminer une longueur, soit la dimension du mètre. Cependant, si la Géométrie non euclidienne était acceptable, le nombre $t$ déterminerait un angle égal à la fraction $t$ d'angle droit, et, suivant que $t$ est inféricur ou supérieur à $\frac{2}{3}$, on saurait construire un triangle équilatéral $A B C$ ayant ses trois angles égaux à $t$ droit, et qui serait ou lobatschewskien ou riemannien; le seul nombre $t$ permettrail donc de tracer la longueur $\mathrm{AB}\left({ }^{2}\right)$.

29. Objection des sphères et pseudo-sphères. - Avant d'entrer dans le fond même de l'argumentation, il importe de faire une remarque générale qui pourrait déjà servir de réponse topique. Il n'y a pas une seule des objections faites

(') Lieu des points $M$ tels que la tangente en chacun d'eux limitée à ce point et à son intersection avec une droite fixe ait une longueur constante.

(2) G. Tarny, Considérations sur le Postulatum d'Euclide, Gauthier-Villars, 1897 .

IRIS - LILLIAD - Université Lille 1 
dans ce paragraphe à la Géomėtric non euclidienne qui ne puisse, avec la mème apparence de raison, se retourner contre les euclidiens eux-mêmes. En premier lieu, la Géométrie des figures tracées sur la sphère est indépendante du $5^{\mathrm{e}}$ postulat d'Euclide, et c'est à Lagrange, paraît-il, que l'on doit attribuer cette observation fondamentale (1); donc, dans tous les espaces géométriques admissibles a priori, la planimétrie de ces figures est réglée par les mêmes lois. Par suite, le plan riemannien pourrait être aussi bien une sphère lobatschewskienne qu'une sphère euclidienne; en fait ce plan est bien une sphère, mais c'est au sens riemannien du mot.

Lobatschewsky a montré que, si le rayon d'une sphère croît au delà de toute grandeur assignable, cette sphère tend vers un état limite qu'il nomme horisphère, et que l'on peut obtenir aussi en faisant tourner un horicycle autour d'un de ses axes; la planimétrie de l'horisphère est jdentique à celle du plan euclidien, les horicycles remplaçant les droites. Si donc un géomètre Iobatschewskien s'avise de prétendre que sa doctrine est la seule d'accord avec la notion de droite, et que le plan euclidien n'est autre chose qu'une horisphère, sur laquelle les horicycles méridiens représentent ce qu'un géomètre euclidien appelle des droites, quel argument valable pourra-t-on lui opposer?

Or, le cas que nous venons de citer n'est point isolé. Si avec les données de la Géométrie euclidienne l'on a pu construire de toutes pièces des surfaces à courbure constante, sphères et pseudo-sphères, sur lesquelles les figures à deux dimensions sont tour à tour analogues aux figures planes riemanniennes ou lobatschewskiennes, cette possibilité s'étendelle d'une façon absolue aux autres espaces admissibles, et, en d'autres termes, avec les données fondamentales de l'une des Géométries non euclidiennes, sait-on aussi construire des surfaces le long desquelles les figures géodésiques à deux dimensions possèdent une planimétrie tour à tour riemannienne, euclidienne ou lobatschewskienne?

La réponse à cette question est affirmative. Dans nos $\dot{E} t u d e s$ de Géométrie analytique non euclidienne, $\$ 42$, nous avons fait voir que les hypercycloïdes de révolution ou canaux, tant

(1) Voir la note (x) de la page 53.

IRIS - LILLIAD - Université Lille 1 
riemanniens que lobatschewskiens, sont des surfaces applicables les unes sur les autres dont la courbure totale égale - I ou + I, et que la somme angulaire d'un quelconque de leurs triangles géodésiques vaut constamment deux angles droits; l'horisphère doit être regardée comme un hypercycloïde limite dont l'axe est indéfiniment éloigné. Nous avons également prouyé qu'il existe des pseudo-sphères riemanniennes ou lobatschewskiennes à courbure constante, tantôt positive, tantôt nulle ( $\left.{ }^{1}\right)$, tantôt négative, le long desquelles un triangle géodésique a toujours une somme d'angles inférieure à deux droits.

Nous compléterons le Tableau de ces surfaces en y ajoutant les hypersphères, qui ont évidemment aussi une courbure constante, et dont la planimétric est riemannienne ou lobatschewskienne suivant leur origine. La parfaite symétrie qu'il nous offre fait ressortir d'une manière saisissante l'indépendance absolue des trois systèmes de Géométrie, qui peuvent chacun tout tirer de son propre fonds sans avoir besoin de rien emprunter aux autres; elle fournit à l'encontre de ceux qui n'ont pas encore renoncé au chimérique espoir de démontrer le postulat des parallèles un argument philosophique de valeur inattaquable.

Entrons maintenant dans la discussion même de l'objection soulevée, et, prenant au pied de la lettre les motifs sur lesquels elle se fonde, répondons :

Sans doute, le géomètre euclidien peut interpréter la Géomètrie riemannienne sur une sphère, mais cela ne lui donne aucun droit à conclure que cètte Géométrie ne fait qu'un avec la Géométrie sphérique usuelle. Nous avons vu en effet, dans le Chapitre II, que la droite est d'une façon générale l'être géométrique caractérisé uniquement dans l'espace par la définition (4) et les postulats I, 2,4 ; en ceci, nulle hésitation; tous les géomètres, euclidiens ou non, sont parfaitement d'accord. Le plan est aussi, d'une façon générale, l'être géométrique caractérisé par les définitions précédentes et la définition (7). Par toute droite on peut faire passer une infinité

(') Le cóne engendré par la révolution d'une droite lobatschewskienne autour d'une de ses parallèles rentre dans ce cas particulier remarquable. 
de plans. Ajoutons-y le rejet du postulat 6 , mais rien de plus, nous aurons défini spécialement la droite riemannienne et le plan riemannien; par toute droite riemannienne on peut donc faire passer une infinité de plans riemanniens, et dans chacun d'eux cette droite a un centre déterminé et distinct; mais on peut concevoir la droite sans les plans.

Or, ce qui précède n'a aucune espèce de sens quand on parle de grand cercle au lieu de droite, et de sphère au lieu de plan. Fn effet, sur la sphère, le grand cercle est bien l'ctre géométrique défini par les conventions (4), r, a, et 4 , et il est prouvé de plus qu'il jouit de la propriété exprimée par le rejet du postulat 6 , mais sa considération ne peut aller sans celle du pseudo-plan unique qui le renferme, et dont il est impossible de l'abstraire, chaque portion si minime qu'elle soit de ce grand cercle le déterminant tout entier, ainsi que le centre et le rayon de la sphère dont il fait partie.

On aura beau appeler à son aide les ressources de l'analyse et les propriétés factices des hyperespaces, et essayer de prouver : que si, dans un espace cuclidien a quatre dimensions ayant la droite euclidienne pour géodésique, l'on considère l'espace sphérique à quatre dimensions

$$
x^{2}+y^{2}+z^{2}+v^{2}=\mathbf{R}^{2}
$$

coupé par l'espace euclidien à trois dimensions $x=0$ suivant la sphère

$$
y^{2}+z^{2}+y^{2}=\mathrm{R}^{2},
$$

la rotation de cette sphère autour du plan des $v z$ dans son espace à trois dimensions engendre l'espace sphérique entier et revient à un retournement de cette même sphère opéré dans cet espace sphérique autour du grand cercle

$$
x=0, \quad y=0, \quad z^{2}+v^{2}=\mathrm{R}^{2} ;
$$

que par suite celte sphère a sur ce grand cercle deux centres opposés avec le même rayon $\frac{I}{2} \pi R$; on ne saurait en conclure que les plans de Riemann sont identiques aux sphères d'Euclide, et que les espaces riemanniens à irois dimensions sont 
des espaces sphériques susceptibles d'entrer dans des espaces euclidiens à quatre dimensions ( ${ }^{1}$ ).

On aura seulement prouvé que lous les espaces sphériques cuclidiens ou non jouissent de propriétés semblables, car, dans les équations précédentes, $x, y, z$, v et $\mathbf{R}$ sont aussi, à condition de les choisir convenablement, des coordonnées et une constante applicables à un espace sphérique de forme quelconque.

La définition d'une circonférence, d'une sphère, d'un espace sphérique de forme euclidienne ne peut se concevoir d'ailleurs sans qu'on y ajoute, implicitement ou non, la considération duccentre et du rayon de cette circonférence, de cette sphère ou de cet espace sphérique; or, il faut distinguer suivant que l'on entend parler des propriétés absolıes de ces figures, ou des propriétés relatives, soit de la circonférence considérée comme grand cercle de la sphère de même rayon, soit de la sphère considèrée comme grande sphère de l'espace sphérique de même rayon.

Dans le premier cas, les figures considérées n'ont qu'un centre, et les distances de ce centre à leurs divers points sont complées suivant des droites euclidiennes; dans le second, ces figures ont deux centres et les distances sont comptées suivant des circonférences. Il en est tout autrement dans Ja Géométrie riemannienne; qu'il s'agisse des propriétés absolues ou des propriétés relatives, la droite, le plan, l'espace ont deux centres symétriques par rapport à ces figures, et les distances de chacun de leurs points à ces centres sont toujours comptées suivant des droites $\left({ }^{2}\right)$.

La distinction entre le plan lobatschewskien et la pseudosphère est encore plus évidente, puisque les géodésiques de cettedernière se coupent deux à deux non en un point, mais en un nombre de points indefini; puisque sur la premicie surface on peut tracer des droites dans tous les sens, tandis qu'on ne peut en faire autant pour les lignes géodésiques de la seconde; puisque enfin une portion quelconque du plan lobatschewskien, qui. est rigide, peut s'appliquer intégrale-

(1) Lforhalas, Ouviages cités.

(2) Nous empruntons ces arguments à M. Massiox : Sur la nonidentité du plan riemannien et de la sphère euclidienne.

Scientia, $\mathrm{n}^{\circ} 15$.

IRIS - LILLIAD - Université Lille 1 
ment sur une autre portion de ce plan par une simple rotation autour d'une droite, tandis qu'une portion de pseudo-sphère ne peut s'appliqưer sur une autre portion de la mêmc surface, par repli autour d'une géodésique, qu'après une cértaine tlexion analogue à celle qu'il faut faire subir à un corps creux en caoutchouc, incomplètement fendu dans sa longoeur, pour superposer les deux parties de sa superficie externe.

30. Objection du triangle équilatéral. - Cet argument est plus spécieux que réel, et il est aisé d'y répondre; car la proposition que voici est d'une vérité absolue :

Il y a une infinité de triangles équilatéraux $\mathrm{ABC}$ où le rapport de l'angle $\mathrm{BAC} \grave{a}$ l'angle droit égale un nombre donné $t$.

Deux cas se présentent selon que $t$ est égal ou inégal à $\frac{2}{3}$. Si $t$ est égal à $\frac{2}{3}$, la proposition est évidente, puisque nous sommes en Géométrie usuelle, el que tous les triangles équilatéraux du plan répondent à la question.

Soit $t$ supérieur ou inférieur à $\frac{2}{3}$. Imaginons un plan non euclidien de paramètre $\mathrm{U}$; il existe sur ce plan un angle $\mathrm{T}$ dont la mesure en angle droit est $t$, et un triangle rectangle déterminé dont les deux angles non droits sont égaux l'un à $T$, l'autre à $\frac{1}{2} T$; le triangle équilatéral demandé est la réunion de deux pareils triangles rectangles, et la longueur de son côté $A B$ est une fouction déterminée de $U$. Fn réalité, le nombre $t$ ne fait connaître que le rapport $\frac{\mathrm{AB}}{\mathrm{U}}$, et à chaque valeur de $U$ correspond un triangle unique satisfaisant à la question.

Si donc, imaginant un triangle équilatéral ABC dont l'angle au sommet $\mathrm{T}$ égale $t$ droit, il est possible du nombre $t$ seul de remonter à la longueur concrète $A B$ que l'on sait effectivement construire sur le plan riemannien ou lobatschewskien donné, c'est que $t$ n'est pas la seule donnée du problème; il y a une autre donnée objective latente, le paramétre $U$ du

IRIS - LILLIAD - Université Lille 1 
plan où les constructions sont censées s'effectuer. On ne peut, quoiqu'on le veuille, en faire abstraction; mais, ne sachant pas la mesurer exactement, on y supplíe par une longueur arbitraire prise pour base des opérations du quadrilatère trirectangle $(\$ 2 / 4)$.

31. Autres objections. - Un certain nombre d'autres objections de moindre valeur on lété faites à la doctrine non euclidienne; si nons en disons un mot, c'est qu'elles touchent par certains côtés à la philosophie même des Sciences.

I. Il lui a été reproché d'abord d'être en contradiction avec la théorie atomique, constamment soutenue par la plupart des grands génies scientifiques, et acceptèe aujourd'hui, d'après ce patronage illustre, d'une façon à peu près universelle: "Quelque temps", affirme Pascal dans l'Esprit géométrique, " quelque mouvement, quelque espace que ce soit, il y en a toujours un plus grand et un moindre, en sorte qu'ils se soutiennent tous entre le néant et l'infini en étant toujours également éloignés de ces extrêmes. "Ou encore : "Toute grandeur mathématique qui décroît passe, avant de cesser d'exister, par un état particulier tel que rien de plus petil que lui n'existe; c'est l'atome ou monade de cette grandeur, qui renferme en substance, et à l'état le plus réduit possible, toute la série des propriétés que l'analyse en déduira par la suite " (Vatson, Les savants illustres, Travaux scientifiques de Leibniz). D'après ces définitions de l'atome, celui-ci ne peut diminuer qu'en disparaissant dans le néant, et augmenter qu'en devenant double, triple, quadruple, etc. Or, si l'on considère le quadrilatère plan ABCD birectangte et isoscèle de notre figare $a$, et qu'on y suppose $A B$ égale à un atome de loaguerr, $A G$ set $B D$ étant queloonques, CD, qui est aussi atome, ne peut être moindre que $A B$ ou surpérieure à $A B$ sans qu'il existe deux perpendicalaires à une droite issues d'an point quelconque; ceci entraîne donc

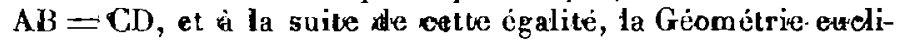
dienre (1).

(1) J. BoNNEL, Les atomes et hypothèses dans la Géométrie, 1899; Note sur Res systèmes de Giométrie et l'atome, Lyon, rgoo.

IRIS - LILLIAD - Université Lille 1 
Le raisonnement serait inattaquable si l'alome CD) était de même espèce que AlB, tandis qu'il n'en est évidemment rien; tout ce qu'on peut dire a priori à son sujet, c'est qu'il est une certaine fonction de $\mathrm{AB}$ et de $\mathrm{AC}$, qui disparait avec $\mathrm{AB}$, ou devient avec lui double, triple, quadruple, etc. Au surplus, la Géométrie atomique d'une surface quelconque, par exemple d'une sphère, devrait toujours être euclidienne!

- II. On a dit encore que tout ce qui est conçu par la raison doit être également imaginable, ou doit pouvoir se représenter soit aux sens, soit à l'imagination; ceci n'est rien moins qu'exact, car notre raison conçit lindélini que notre imagination est impuissante à se représenter; car, ainsi que le dit très à propos M. Mansion ( ${ }^{1}$ ), nous ne pouvons nous imaginer ni la façon dont varie la courbure d'une cycioïde aux environs de son point de rebroussement ni la courbe continue de Weierstrass, qui n'a de tangente en sucun point, ni la courbe continue de M. Peano qui a tous ses points à des cotes différentes entre zéro et I, et dont la projection remplit estièrement un carré de côté égal à r Notre imagination est une '́aculté tellement débile que, sans l'aide de la raison, elle ne pourrait mème pas se représenter cent points physiques marqués sur une feuille de papier; tout a ulus peutelle constater qu'il y en a un assez grand nombre. Du reste, ajoute en terminant le savant professeur, il est impossible pour nous d'atlacher aux notions de droite finie, de plan fini, d'espace fini, l'image d'une forme précise quelconque, parce que, si le monde est riemannien, nous en faisons partie, et nous ne pouvous nous isoler même par la pensée de ce moude pour nous en faire une représentation sensible.

III. Enfin, on a prétendu, en se basant sur l'autorité de Duhamel $\left({ }^{2}\right)$, que l'idée simple de deux droites partout également distantes devait êlie considérée comme innée puisqu'elle se présente à l'enfant, et que d'ailleurs nous en avons continuellement des exemples sous les yeux. Or, d'après ce que nous avons vu à l'article 5 du paragraphe 21 , il y a deux systèmes de Géométrie qui contiennent de telles droites, seule-

(') Pour la Géométrie non euclidienne (Mathesis, 1898, p. 37-38).

(2) Des méthodes dans les Sciences de raisonnement, ${ }^{2}$ Partie.

IRIS - LILLIAD - Université Lille 1 
ment, dans l'un elles appartiennent au même plan, tandis qu'il n'en est pas de mème dans l'autre; il n'y a donc aucun moyen théorique de prouver que ce oni peut seulement étre existe véritablement.

To be, or not to be, that's the question.

(Shakspeare, Hamlet, acte III, scène r.)

32. L'impossibilité de démontrer le Postulatum d'Euclide. L'échec des innombrables tentatives faites depuis si longtemps pour donner du Postulatum d'Euclide une démonstration à l'abri de toute crilique éveille dans l'esprit le doute le plus absolu sur l'existence de cette démonstration, mais ne constitue pas une preuve scientifique de son inexistence. Cette preuve découle au contraire rigoureusement des résultats analytiques ou géométriques que voici :

$1^{\circ}$ Établissement des formules de la Trigonométrie générale en partant du conceut fondamental de distance; ceci montre que le postulat euclidien est indépendant des premiers fondements de la Géométrie, définition de la droite el du plan, congruence.

$2^{\circ}$ Interprétation, dans une région normale, soit de la Géomitric non cuclidienne sur une surface à courburc constante euclidienne, soit de la Géométrie euclidienne sur une surface à courbure constante non euclidienne.

3o Existence de systèmes analytiques non euclidiens, d'après les idées de hiemann, Helmholtz ( $\left.{ }^{1}\right)$ et les recherches de Lie $\left({ }^{2}\right)$ sur les groupes de transformations; ces dernières montrent qu'il y a dans l'espace euclidien des groupes de transformations analogues aux mouvements dans l'espace non euclidien.

$4^{\circ}$ Enfin, représentation, par la méthode projective de

(') Hezmolтz, 1821-189'. Ueber die thatsächlichen Grundlagen der Geometrie, r868, traduction française de Hoüel dans les Mémoires de Bordeaux, 868 ; The axioms of Geometry (Revue des cours scientifiques, I870); Veber die Axiome der Geometrie (Revue scientifique, $1877)$.

(2) Sophus LiE, ${ }^{2} 84^{2-1} 899$. Theorie der Transformationsgruppen, Lcipzig, Teubner, 1893 .

IRIS - LILLIAD - Université Lille 1 
8o LES CONTRADIETEURS DE LA GÉOMÉThIE NON EUCLIDIENNE.

Cayley $\left({ }^{1}\right)$, de l'espaoe non euclidien dans l'espace euclidien. Par exemple, soit $S$ un cercle de ce dernier appeié l'absolu; la région intérieure est le plan, une corde du cercle est une droite; si la corde qui joint deux points: $\mathrm{A}$ et $\mathrm{B}$ rencontre l'absolu en $P$ et $Q$, le logarithme du rapport anharmonique $\mathrm{APBQ}$ est la distance $\mathrm{AB}$, enfin la distance des pôles de deux droites par rappert à l'absolu est l'angle de ces droites. Moyennant ces conventions, la Géométrie de la région intérieure est celle du plan de Lobatschewsky, et il est aisé de l'étendre à l'espace en substituant une sphère absolue au cercle absolu.

(1) Sixth Memoir upon Quantics (Philosophical Transactions, r859). Voir a ussi KLEIx, Ueber die Sogennante nicht euklidische Geometrie (Math. Annalen, $8_{7} \mathrm{x}$ ). 


\section{CHAPITRE VIII.}

LA GÉOMÉTRIE PHYSIQtE.

33. La forme géométrique de notre univers. - En Physique, en Cristallographie, et dans les Arts, on considère des lignes et des surfaces qui réalisent aussi parfaitement que nos sens permettent de le constater les définitions générales de la droite et du plan. La Géométrie de ces lignes et de ces surfaces paraît régie par les lois euclidiennes; par exemple, dans les plus grands triangles rectilignes dont il ait été possible de mesurer dircetement les angles, la somme de ceux-ci a toujours été à peine différente de deux droits.

Notre univers est-il donc véritablement euclidien, et faut-il mettre uniquement au compte des imperfections de nos instruments de mesure l'écart, si faible qu'il soit, que nous constatons entre les résultats théoriques et ceux de l'expérience? Il est permis d'en douter.

Du moment que le système de Géométrie cherché ne présente aucun caractère de nécessité $a$ priori, la réserve la plus complète ne peut que s'imposer quant aux conclusions à tirer de nos mesures. D'après le philosophe Reid ( $\left.{ }^{1}\right)$, l'homme réduit au simple sens de la vue, et ne pouvant connaître que l'étendue superficielle à deux dimensions, prendrait pour des droites ce qui serait réellement des arcs de grand cercle tracés sur une surface sphérique dont son oil occuperait le centre; pour un tel homme, évidemment, le monde physique serait bâti suivant la conception riemannienne.

Imaginons maintenant, avec M. H. Poincaré $\left({ }^{2}\right)$, une

( $\left.{ }^{1}\right)$ Voir AMPÉRE, Essai sur la philosophie des Sciences.

(2) Revue générale des Sciences pures et appliquées, $189^{2}$, p. 7). Voir aussi les Leçons de Géométrie de M. J. HADAMard, Note II.

IRIS - LILLIAD - Université Lille 1 
sphère S limitant un milieu dont l'indice de réfraction et la température soient variables, et dans ce milieu, des objets mobiles dont les déplacements soient assez lents et les chaleurs spécifiques assez faibles pour qu'ils se mettent immédiatement en équilibre de température avec le milieu. Si ces objets ont le même coefficient de dilatation, la longueur de l'un quelconque d'entre eux peut définir la température. $\Lambda d$ mettons que $R$ désignant le rayon de la sphère, et $\hat{r}$ la distance d'un point du milieu au centre, la température absolue et l'indice de réfraction soient mesurés respectivement en ce point par $\mathrm{R}^{2}-\rho^{2}$ et $\frac{\mathrm{T}}{\mathrm{R}^{2}-\rho^{2}}$; si des êtres intelligents habitent un semblable monde, ils croiront nécessairement : ${ }^{\circ}$ que les dimensions des objets mobiles n'ont pas changé puisqu'elles ont varié dans le même rapport: $2^{\prime \prime}$ que la sphère $\mathrm{S}$ a un rayon infini, car, plus ces objets s'approcheront de la péripherie, plus leurs mouvements seront lents par suite du refroidissement qu'ils éprouvent; $3^{\circ}$ que les circonférences orthogonales à la sphère $\mathbf{S}$ sont des droites, puisque ce sont les trajectoires des rayons lumineux, et d'ailleurs les ohjets situés hors de la sphère $S$ demeurent invisibles : $4^{\circ}$ que dans un triangle rectiligne la somme des angles est moindre que deux droits, puisque c'est là mème une propriété des triangles curvilignes formés par trois cercles orthogonaux à $S$. En conséquence, ces êtres ne pourront adopter que la Gréométrie de Lobatschewsky ( ${ }^{1}$ ).

Mais la réalité des faits concorde aussi bien avec un système physique riemannien ou lobatschewskien de paramètre très grand qu'avec le système euclidien. Nous avons démontré dans le paragraphe 27 que ce delnier pouvait ètre regardé comme un cas limite des deux autres, et d'ailleurs les formules (7) et (8) du paragraphe $\mathbf{2 3}$ reviennent à une formule unique équivalente au fond à la relation III du paragraphe 15 , pourvu qu'on suppose aiternativement $\lambda$ égal à $\mathbf{~}$ ou à $\sqrt{-1}$,

(1) Lire D. Hilbent : Fondements de la Geometrie, paragraphe 10, et un extrait de lettre de cet auteur à M. KLEIN dans IFns. math., igor, p. r9f et suiv. Consulter aussi M. F. Pletzken : La forme de l'espace, $18 \mathrm{gr}$, et Considérations sur la forme de l'espace (Ens. mall, 1902$)$. 
c'est-à-dire $\varepsilon$ égal à r ou $\dot{a}-\mathbf{I}$. Ces faits analytiques font ressortir avec la plus heureuse simplicité le caractère philosophique de la Métagéométrie, et expliquent l'absolu et inévitable insuccès de tous les efforts tentés ou a tenter pour prouver théoriquement la vérité du postulat 5. L'expérience seule devrait lever les doutes, mais a la condition absolue que ses opérations eussent lá précision théorique; donc, à supposer que notre univers fût exactement euclidien, il nous serait impossible, vu l'imperfection relative de nos procédés de mesure, de le démontrer expérimentalement. Au contraire, nous allons voir que, si nos déterminatious de droites, de longueurs, d'angles finissaient par s'effectuer avec une exactitude suffisante, peut-être un jour aurions-nous le moyen de savoir si notre monde physique est riemannien ou lolsatschewskien, en calculant le signc de son paramètre, et mème, dans le cas nù ce paramètre serait assez petit, sa grandeur approchée.

34. Mesures relatives au paramétre. - Supposons que l'on soit parvenu à dessiner très exactement sur une planchette d'ingénieur aussi bien nivelée que possible un quadrilatère trirectangle $A B C D$ dont les côtés $B C$ et $C D$, adjacents aux angles droits $B, C, D$ aient ${ }^{\prime} m$ de longueur. Ceci constitue déjà une opération graphique très délicate à exécuter malgré a simplicité théoriqque. L'angle $A$, mesuré au cercle répétiteur, a été trouvé égal a go avec une incertitude en plus ou en moins comprise entre $\frac{1}{10}$ el $\frac{1}{100}$ de seconde $\left({ }^{1}\right)$.

Si le paramètre fini $U$ de l'espace non euclidien est exprimé en mètres, les équations

$$
\sin ^{2} \frac{\mathrm{I}}{\mathrm{U}}=-\cos \mathrm{A}, \quad \operatorname{sh}^{2} \frac{\mathrm{I}}{\mathrm{U}}=\cos \mathrm{A}
$$

établissent la dépendance analytique qui doit exister entre ce paramètre et l'angle $A$. Or, il suffit que le paramètre dépasse seulement jooo $^{\mathrm{m}}$ pour que la valeur calculée de $\mathrm{A}$ diffère de $90^{\circ}$ de moins de $\frac{1}{100}$ de seconde. Ainsi, les conditions phy-

(') A dessein, nous exagérons ici d'une façon notable les limites de lapproximation qui est dans nos moyens actuels; notre raisonement n'en a que plus de force.

Scientia, n* 15.

IRIS - LILLIAD - Université Lille 1 
siques dans lesquelles notre univers est construit nous permettent d'affirmer que son paramètre est, sinon infini, du mains considérable, et, jusqu'à ce jour du moins, nos mesures directes comportent, malgré le soin que nous pouvons $y$ apporter, une somme d'erreurs bien supérieure à l'écart que produirait, dans les mêmes cas, le passage d'un système de Géométrie à l'autre. Par exemple, dans sa Pangéométrie, Lobatschewsky base sur la connaissance de la parallaxe d'une étoile un calcul astronomique destiné à lui donner une limite inférieure de ce paramètre. Dans le triangle rectangle $A B C$, où A représente l'étoile, et BC le grand axe de l'orbite terrestre, l'angle de parallélisme correspondant à $B C$ est plus grand que le complément de l'angle aigu $\mathrm{A}$ égal à la parallaxe, done $\left(\$ 24,1^{\circ}\right)$

$$
\cos I(B C)=\operatorname{th} \frac{B C}{U}<\sin A
$$

ou

$$
\frac{B C}{U}<e \operatorname{tang}\left(45^{\circ}+\frac{A}{2}\right)
$$

En appliquant cette méthode à l'étoile polaile dont la parallaxe vaut à peu près $o^{\prime \prime}, 1$, on tronve que le paramètre $U$ dépasse zoooos fois le diamètre de l'orbite. II faut en conclure qu'en fait, dans la partie de l'univers que nous pouvons directement aborder, la Géométrie est aussi bien non euclidienne qu'euclidienne; mais, comme pratiquement les résultats ne diffèrent pas, il y a avantage à lui conserver le second caractère oì les considérations tank synthétiques qu'analytiques sont plus simples.

Pour être fixé immédiatement sur le choix à faire, il faudrait, par exemple, que trois observateurs situés sur trois planètes différentes pussent se viser réciproquement à un instant donné, et connaitue les angles de déviation de leurs lunettes. Mais, sans attendre cet événement chimérique, il n'est pas interdit d'espérer, grâce aux progrès rapides de la Mécanique moderne, que les appareils d'observation soient en mesure d'acquéric par la suite une beaucoup plus grande perfection. Si ceci se réalise, l'on pourra utiliser une donnée géométrique très simple mise en évidence dans le cours du paragraphe 27.

IRIS - LILLIAD - Université Lille 1 
Nous y avons vu effectivement que la projection d'un segment de droite sur un axe peut être supérieure, ėgale ou inférieure au produit du segment par le cosinus de son inclinaison. Par conséquent, sous une inclinaison de 6o", la projection est supérieure, égale ou inférieure à la moitié du segment. Traçons six rayons indéfinis à $60^{\circ}$ les uns des autres, et portons-y successivement les longueurs $\mathrm{OA}_{0}, \mathrm{OA}_{1}, \mathrm{OA}_{2}$, $\ldots, \mathrm{OA}_{n}$ dont chacune est la projection de la suivante. Suivant que la Géométrie est riemannienne, euclidieane ou lobatschewshienne, on a

$$
\mathrm{OA}_{n} \lesseqgtr 2^{n} \cdot \mathrm{OA} \text {. }
$$

Donc, si par suite d'opérations répétées on finissait par trouver entre $\mathrm{OA}_{n}$ et $2^{n} \mathrm{OA}_{0}$ une différence de sens constant, et plus considérable que la limite supérieure des erreurs imputables aux appareils, le doute serait levé : l'univers ne serait pas euclidien, et le sens de la différence ferait savoir de quelle manière.

Or, à l'époque que nous souhaitons, les mécaniciens ne seraient peut-être pas erubarrassés pour construire une circonférence de très grand rayon. Concevons un arc $l$ de cette ligne, correspondant à quelques secondes, et en tous cas assez faible pour pouvoir être assimilé à une portion de la tangente en l'une de ses extrémités $A$, puis, sur le prolongement $\mathbf{A X}$ du rayon $O A$, prenons les points $B$ et $B^{\prime}$ tels que $A B=l$, et. $\mathrm{AB}^{\prime}=\frac{1}{2}$ l. Si nous mesurions les angles $a=\mathrm{ABT}, x^{\prime}=\mathrm{AB}^{\prime} \mathrm{T}^{\prime}$ que font avec $O X$ les tangentes $B^{\prime} T$ et $B^{\prime} T^{\prime}$ menées des points B et $B^{\prime}$ à la circonférence, deux équations du second degré,

$$
\begin{gathered}
\left(\sin ^{2} x^{\prime}-\sin ^{2} \alpha\right) x^{2}+2 \sin ^{2} x^{\prime} x+\sin ^{2} \alpha^{\prime}-3=0 \\
y^{2}=\left(1+\sin ^{2} \alpha\right) x^{2}-2 x
\end{gathered}
$$

détermineraient en fonction de $\alpha$ et de $\alpha^{\prime}$ la mesure trigonamétrique $y$ de l'arc $l$, et par $x$ la mesure naturelle $r=\frac{\mathrm{R}}{\mathrm{U}} \mathrm{du}$ rayon de la circonférence, qui a son cosinus circulaire ou hyperbolique égal à $\frac{x-1}{y}$. La longueur AB étant, par exemple, de $n$ mètres, les expressions de $\mathbf{L}$ et de $R$ en mètres 
seraient alors respectivement égales soit à $\frac{n}{y \sin r}$ el $\frac{n r}{y \sin r}$ dans le cas d'un univers riemannien, soit au contraire à $\frac{n}{y \operatorname{shr}}$ et $\frac{n r}{y \operatorname{shr}}$ dans le cas d'un univers lobatschewshien.

Le résumé et la conclusion de notre Travail doivent être un hommage rendu premièrement au génie d'Euclide, qui a su choisir avec une si admirable sagacité les postulats fondamentaux de sa Géométrie, deuxièmement aux recherches patientes de Lobatschewshy, Bolyai, Riemann et leurs disciples, recherches qui, en dévoilant la vraie nature de ces mêmes postulats, ont permis de créer à côté du système usuel deux autres systèmes également admissibles au point de vue de la rigucur logique, d'assenil sur des bases inebranlables l'édifice de la Géométrie générale, et par conséquent enfin de consolider définitivement. l'œuvre même du géomètre grec. 


\section{NOTE.}

SCR DELX QUADRJLATÉRLS BIRECTAXGLES ET ISOSCÈLES

DE LA RÉgION NORMaLE.

Thlontme I. - Si deux birectangles isoscèles ont mêmes bases el des hauteurs différentes, les angles aux sömmets sont ensemble droits, aigus ou obtus.

Considérons Jes birectangles $a b \mathrm{BA}$ et $a b \mathrm{~B}^{\prime} \mathrm{A}^{\prime}$ de la fignure ı tirons la médiatrice commune $c \mathrm{C}^{\prime} \mathrm{C}$ de $a b, \mathrm{~A}^{\prime} \mathrm{B}^{\prime}$ et $\mathrm{AB}$, puis traçons $A_{1} B_{1}$ perpendiculaire à $c C$ en son milieu $C_{1} ;$ le repli de la figure suivant $A_{1} B_{1}$ amène $A$ en l'un des points $a, \alpha$ ou $\beta$,

Fig. 15.

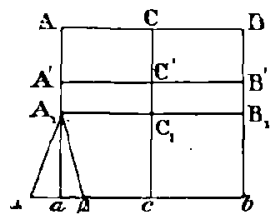

Fig. If.

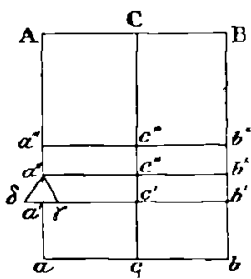

selon que l'angle $a A_{1} C_{1}$ est droit, aigu ou obtus, et réciproquement. Cet angle est donc de même nature que l'angle $a \mathrm{AC}$, et la proposition est prouvée lorsque $c \mathrm{C}^{\prime}$ égale $\frac{\mathrm{I}}{2} c \mathrm{C}$, c'està-dire plus généralement lorsque $c \mathrm{C}^{\prime}$ égale $\frac{\mathrm{r}}{\mathrm{x}^{n}} c \mathrm{C}$. En mème temps, AC est égale, supérieure ou inférieure à $a c$ et réciproquemeat.

Admettons maintenant que la proposition soit vraie quand $c C^{\prime}$ égale $\frac{p}{2^{n}} c C, p$ désignant l'un quelconque des entiers inférjeurs ou au plus égaux à $m<2^{n}$. Prenons dans la figure 16

IRIS - LILLIAD - Université Lille 1 
$c c^{\prime \prime}=-\frac{m}{2^{n}} c \mathrm{C}$ et $c c^{\prime \prime \prime}=\frac{m+-1}{2^{n}} c \mathrm{C}$; l'angle $a \alpha^{\prime \prime} c^{\prime \prime}$ est droit, aigu ou obtus en même temps que A par hypothèse, donc le repli. du quadrilatère $a^{\prime \prime} b^{\prime \prime} b^{\prime \prime \prime} a^{\prime \prime}$ autour de $a^{\prime \prime} b^{\prime \prime}$ amène respectivement dans les mêmes cas le point $a^{\prime \prime \prime}$ à coüncider aver les points $a^{\prime}$, ô ou $\gamma$; par conséquent, $a^{\prime \prime \prime} c^{\prime \prime \prime}$ est éģale, supérieure ou inférieure à $a^{\prime} c^{\prime}$ et à $a c$, c'est-à-dire que l'angle $a a^{\prime \prime \prime} c^{\prime \prime}$ est, en même temps que $\mathrm{A}$, droit, aigu ou oblus. Alors la proposition subsiste quand $c \mathrm{C}^{\prime}$ égale $\frac{m+1}{\mathbf{2}^{n}} c \mathrm{C}$, ou quand $c \mathrm{C}^{\prime}$ égale $\frac{p}{2^{n}} c \mathrm{C}, p$ étant un des entiers quelconques moindres que $2^{n}$. Elle est donc vraie également lorsque l'on a

$$
\frac{p}{2^{n}}<\frac{c \mathrm{C}^{\prime}}{c \mathrm{C}}<\frac{p+1}{2^{n}}
$$

$n$ croissant indéfiniment, et par suite elle est générale.

Théreme II. - Si deux birectangles isoscèles ont mêmes hauteurs et des bases différéntes, les angles aux sommets sonl ensemble droits, aigus ou oblus.

Considérons les deux birectangles $a b \mathrm{BA}$ et $a b^{\prime} \mathrm{B}^{\prime} \mathrm{A}$ accoles suivant aA dans la figure 17 , ainsi que la médiatrice c $\mathrm{C}$ de $a b$ et $\mathrm{AB}$. L'extrémité d'une longueur égale à $a \Lambda$ et portée

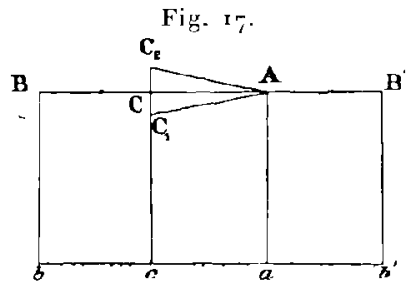

sur $c C_{1}$ tombe en $C_{2}, C_{2}$ ou $C_{1}$, suivant que l'angte $a A B$ est droit, aigu ou abtus; donc en même temps on a respectivement les relations

$$
\begin{aligned}
& a \mathrm{AC}=c \mathrm{C}_{\mathrm{A}}=\mathrm{I} \text { droit, } \\
& \boldsymbol{a A \mathrm { C } _ { 2 }}=c C_{2} \mathrm{~A}<\mathrm{d} \text { droit, } \\
& \boldsymbol{a A \mathrm { C } _ { 1 }}=c \mathrm{C}_{1} \mathbf{A}>\mathrm{I} \text { droit. }
\end{aligned}
$$

IRIS - LILLIAD - Université Lille 1 
Ceci posé, admettons d'abord que $a b^{\prime}$ égale $a c$ ou $\frac{\mathrm{T}}{2} a b$; la proposition est prouvée par ce qui précède, elle l'est donc aussi quand $a b^{\prime}$ égale $\frac{a b}{2^{n}}$. Pour passer au cas général, imaginons que sur chacune des divisions de $a b$ en $2^{n}$ parties égales nous ayons construit un birectangle de hauteur égale à $a \mathrm{~A}$; leurs sommets successifs forment une ligne brisée régulière $\mathrm{AA}_{1} \mathrm{~A}_{2} \ldots \mathrm{A}_{p} \ldots \mathrm{A}_{2 n-1} \mathrm{~B}$ qui est droite, convexe vers $a b$, ou convexe vers l'opposé de $a b$, selon que l'angle $a A B$ est droit, aigu ou obtus.

Lorsque $a b^{\prime}$ égale $\frac{p}{2^{n}} a b$, l'angle $a \mathrm{AB}^{\prime}$ égale l'angle $a \mathrm{AA}_{p}$, et comme ce dernier est toujours compris entre les angles de même nature $a \mathrm{AA}_{1}$ et $a \mathrm{AB}$, à moins qu'il ne coïncide avec eux, il est encore, ainsi que $a \Lambda B$, droit, aigu ou obtus. Enfin, lorsque l'on a

$$
\frac{p}{2^{n}}<\frac{a b^{\prime}}{a b}<\frac{p+1}{2^{n}}
$$

$n$ croissant indéfiniment, il en est encore de même, et le théorème est toujours vrai.

THEonìme III. - Les angoles aux sommets de deux birectangles isoscèles sont ensemble droits, aigus ou obtus.

Car, d'après les deux théorèmes précédents, ces angles sont de même nature que l'angle au sommet du birectangle isoscèle qui a sa base égale à celle du premier birectangle donné, et sa hauteur égale à celle du second.

Thtork̀me IV. - Si dans un birectangle isoscèle l'angle au sommet est droit, aigu óú obtus, dans tout triangle la somme angulaire est égale, inférieure ou supérieure à deux droits.

En effet, soit $\propto$ l'angle au sommet du birectangle isoscèle dunné. La somme angulaire $2 \Sigma$ d'un triangle quelconque ABC est égale, d'après la proposition II de la page 60 à laquelle nous prions le lecteur de vouloir bien se reporter, a double de l'angle $\mathrm{A}^{\prime} \mathrm{BE}$ du quadrilatère birectangle isoscèle BECF construit sur la figure 6. Mais, en vertu du théo- 
rème III de notre Note.' l'angle $\mathrm{A}^{\prime} \mathrm{BE}$ est, en même temps que $\alpha$, droit, aigu nu rins suivant l'hypothèse; donc $2 \Sigma$ est aussi, selon l'hypothèse, égale, inférieure ou supérieure à deux droits.

Conolankrs. - ${ }^{\circ}$ Si dans un seul triangle la somme angulaire surpasse deux droits, elle la surpasse aussi dans tous les triangles, et ceci doit entraîner le rejet du postulat 6 (voir p. 26), c'est-à-dire le premier théolème de Legendre.

$2^{\circ}$ Si dans un seul triangle la somme angulaire est égale, inférieure ou supérieure à deux droits, il en est de mème dans tous les triangles, ce qui prouve le second théorème de Legendre généralisé.

Méthode de M. Bonola. - Pour démontrer la proposition sans l'aide du postulat d'Archimède et du principe de continuité, voici le moyen employé par l'auteur. Considérons le quadrilatère birectangle et isoscèle $a \mathrm{AB} b$; prenons un point $\mathrm{C}$ de $\mathrm{AB}$, un point $\mathrm{D}$ de son prolongement, et abaissons $\mathrm{C} C$ et $\mathrm{D} d$ perpendiculaires sur $a b$ ( $f g$. I 8$)$.

$\mathrm{I}^{\circ}$

$$
\begin{aligned}
& \text { Si } c \mathrm{C}=a \mathrm{~A}, \quad \text { ou } d \mathrm{D}=a \mathrm{~A}, \quad a \mathrm{AB}=\mathrm{I} \text { droit, } \\
& \text { si } c \mathrm{C}<a A \text {, ou } d \mathrm{D}>a \mathrm{~A}, \quad a \Lambda \mathrm{B}<\mathrm{i} \text { droit, } \\
& \text { s1 } c \mathrm{C}>a \mathrm{~A}, \text { ou } d \mathrm{D}<a \mathrm{~A}, \quad a \mathrm{AB}>\mathrm{I} \text { droit. }
\end{aligned}
$$

Fig. 18.

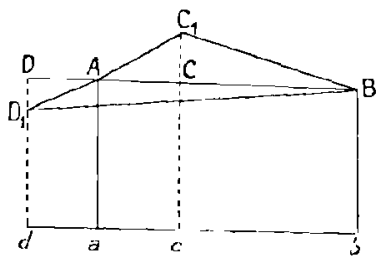

Prenons en effet $c \mathrm{C}_{1}$ et $d \mathrm{D}_{1}$ égaux à $a \mathrm{~A}$; dans la première hypothèse $C_{1}$ et $D_{1}$ coïncident avec $C$ et $D$; donc,

$$
a \mathrm{AC}+b \mathrm{BC}=c \mathrm{CA}+c \mathrm{CB}=2 \text { droits, }
$$

par suite, $a \mathrm{AB}$ est droit; ou

$$
a \mathrm{AD}=a \mathrm{AB},
$$

ce qui conduit au même résultat.

IRIS - LILLIAD - Université Lille 1 
Dans la seconde lyypothèse, $C_{1}$ est sur le prolongement de $c \mathrm{C}$, tandis que $\mathrm{D}_{1}$ est sur $d \mathrm{D}$. 'Tirons $\mathrm{C}_{1} \mathrm{~A}, \mathrm{C}_{1} \mathrm{~B}, \mathrm{D}_{1} \mathrm{~A}$ et $\mathrm{D}_{1} \mathrm{~B}$. La somme des angles $c$ CA et $c \mathrm{CB}$, ou deux droils, est plus grande que la somme des angles $c \mathrm{C}_{1} \mathrm{~A}$ et $c \mathrm{C}_{1} \mathrm{~B}$, c'est-à-dire que la somme des angles $a \mathrm{AC}_{1}$ et $b \mathrm{BC}_{1} ;$ a fortior $i$ est-elle plus grande que $2 a \mathrm{AB}$, donc $a \mathrm{AB}$ est aigu.

L'angle $\mathrm{DAD}_{1}$ est plus grand que l'angle $\mathrm{DBD}_{1}$, et l'angle $a \Lambda D_{1}$ égal à $d D_{1} \mathrm{~A}$ est plus grand que l'angle $b \mathrm{BD}_{1}$ égal à $d \mathrm{D}_{1} \mathrm{~B}$; donc on a

ou

$$
a \mathrm{AD}-\mathrm{DAD}_{1}>a \mathrm{AB}-1 \mathrm{BD}_{1},
$$

$$
2 \text { droits }-2 a A B>D_{1} D_{1}-\mathrm{DBD}_{1} \text {; }
$$

par suite, a fortiori, $a \mathrm{AB}$ est encore un angle aigu.

Enfin, dans la troisième hypothèse, $\mathrm{C}_{1}$ est sur $c \mathrm{C}$, tandis que $\mathrm{D}_{1}$ est sur $d \mathrm{D}$ prolongé; en raisonnant d'une façon analogue, on arrive toujours à la conclusion que l'angle $a \mathrm{AB}$ est obtus.

$2^{\circ}$ Les réciproques des propositions $1^{\circ}$ sont vraies. - On les démontre aisément par la réduction à l'absurde.

Revenons maintenant aux théorèmes I, Il et III de la méthode précédente.

Si dans la figure i 5 l'angle a AC est droit, aigu ou obtus, $c \mathrm{C}^{\prime}$ étant moindre que $c \mathrm{C}, \mathrm{A}^{\prime} \mathrm{C}^{\prime}$ est égale, inférieure ou supérieure à $\mathrm{AC}$; donc l'angle $a \mathrm{~A}^{\prime} \mathrm{C}^{\prime}$ est aussi droit, aigu ou obtus (Théorème I).

Si dans la figure 17 on suppose que $a c$ egale $a b^{\prime}$, fraction quelconque de $a b$, l'extrémité d'une Jongueur égale à $a$ l portée sur la perpendiculaire en $c$ tombe encore en $C, C_{2}$ ou $\mathrm{C}_{1}$ suivant que l'angle $a \mathrm{AB}$ est droit, aigu ou obtus; donc l'angle $c$ CA est, dans les mêmes conditions, droit, aigu ou obtus. Quand il est droit, il en est de mème de l'angle $a \mathrm{AB}^{\prime}$ son égal; quand il est aigu, il en est de même a fortiori de l'angle $c \mathrm{C}_{2} \mathrm{~A}$ et par suite de l'angle $a \mathrm{AB}^{\prime}$; enfin, quand il est obtus, il en est de mème a fortiori de l'angle $c \mathrm{C}_{1} \Lambda$ ou de $a \mathrm{AB}^{\prime}$ ( Théorème II). 
PARIS. - IMPRIMERTE GAUTHIER-VILLARS, 39556 Quai des Grands-Augustins, jō.

IRIS - LILLIAD - Université Lille 1 